\title{
Nuclear and Particle Astrophysics ${ }^{\dagger}$
}

\author{
Norman K. Glendenning \\ Nuclear Science Division \\ Lawrence Berkeley Laboratory \\ University of California \\ 1 Cyclotron Road \\ Berkeley, California 94720
}

October 31, 1990

\author{
Invited Course \\ 1990 International Summer School on the Structure of \\ Hadrons and Hadronic Matter, \\ Dronton, The Netherlands \\ A ugust 5 - 18, 1990
}

\footnotetext{
TTo be published in the Proceedings of the 1990 International Summer School on the Structure of Hadrons and Hadronic Matter, Dronton, The Netherlands August 5 - 18, 1990, by World Scientific. This work was supported by the Director, Office of Energy Research, Office of High Energy and Nuclear Physics, Division of Nuclear Physies, of the U.S. Departnent of Energy under Contract DE-AC03-76SF00098.
} 


\title{
Nuclear and Particle Astrophysics ${ }^{\dagger}$
}

\author{
Norman K. Glendenning \\ Nuclear Science Division \\ Lawrence Berkeley Laboratory \\ University of Californio \\ 1 Cyclotron Road \\ Berkeley, California 94720
}

\begin{abstract}
We discuss the physics of matter that is relevant to the structure of compact stars. This includes nuclear, neutron star matter and quark matter and phase transitions between them. Many aspects of neutron star structure and its dependance on a number of physical assumptions about nuclear matter properties and hyperon couplings are investigated. We also discuss the prospects for obtaining constraints on the equation of state from astrophysical sources. Neutron star masses although few are known at present, provide a very direct constraint in as much as the connection to the equation of state involves only the assumption that Einstein's general theory of relativity is correct at the macroscopic scale. Supernovae simulations involve such a plethora of physical processes including those involved in the evolution of the precollapse configuration, not all of them known or understood, that they provide no constraint at the present time. Indeed the prompt explosion, from which a constraint had been thought to follow, is now believed not to be the mechanism by which most, if any stars, explode. In any case the nuclear equation of state is but one of a multitude of uncertain factors, and possibly one of the least important. The rapid rotation of pulsars is also discussed. It is shown that for periods below a certain limit it becomes increasingly difficult to reconcile thein with neutron stars. Strange stars are possible if strange matter is the absolute ground state. We discuss such stars and their compatibility with observation.
\end{abstract}

${ }^{1}$ Copyright of these lecture notes is reserved by the author and by his sponsor, DOE. The author nuay separately publish, or expand upon and publish these notes as a separate book. This right is not waived by permiting the organizers of the Dronton Summerschool to have the notes published as a part of its proceedings.

This work was supported by the Director, Office of Energy Research, Office of Iligh Energy and Nuclear Physics, Division of Nuclear Physics, of the U.S. Department of Energy under Contract DE-AC03-76SF00098. 


\section{Contents}

1 Preliminaries 2

2 Why Pulsars are Neutron Stars $\quad 8$

3 Chemical Potentials and Phase Equilibria $\quad \mathbf{1 0}$

3.1 Chemical Equilibrium. . . . . . . . . . . . . . . . 11

3.2 Phase Equilibrium $\ldots \ldots \ldots \ldots 12$

4 Quark Matter 14

4.1 Zero Temperature . . . . . . . . . . . . . . . . . . . 15

4.2 Massless Quark Approximation . . . . . . . . . . . 16

4.3 Quark Star Matter ... . . . . . . . . . . . . . . . 17

4.4 Strange and Charm Stars . . . . . . . . . . . . . . 19

5 Nuclear and Neutron Star Matter $\quad 21$

6 Pion and Kaon Condensation $\quad 30$

7 Limiting Neutron Star Mass and the Equation of state 31

8 Supernovae $\quad \mathbf{3 4}$

8.1 General Features . . . . . . . . . . . . . . . 35

8.2 Prompt Bounce and Ejection $\ldots \ldots \ldots \ldots \ldots \ldots \ldots$

8.3 Neutrino Reheating Explosion ............... 38

9 Vacuum Polarization and Neutron stars $\quad 39$

10 Other Dependences of Neutron Stars $\quad 45$

11 Redshift, Binding etc. $\quad 50$

12 Summary of Constraints on the Equation of state 51

13 Stars with Quark Cores: Hybrids $\quad 54$

14 Fast Rotation of Pulsars and the Equation of state 56

15 Fast Pulsars, Strange Stars $\quad 57$

15.1 Bias against short periods of present pulsar surveys. . . . . . . . 58

15.2 Difficulty in reconciling fast pulsars with neutron stars. . . . . . . 59

15.3 Strange matter as the absolute ground state. . . . . . . . . . 62

15.4 Strange quark stars. . . . . . . . . . . . . . . 63

15.5 Strange matter compatible with terrestrial searches. . . . . . . 65 
15.6 Motivation for submillisecond pulsar searches. . . . . . . . . 66

15.7 Accelerator searches for strange nuggets. . . . . . . . . . . 67

16 Appendix 67

16.1 Stress-Energy . . . . . . . . . . . . . . . . 67

16.2 Schwarzschild Solution . . . . . . . . . . . . . . . . 68

16.3 Stable Star Configurations . . . . . . . . . . . . 70

\section{Preliminaries}

Particle physics, nuclear physics of dense matter and astrophysics are connected through Einstein's theory of general relativity. Having derived the special theory, in which time and space were intimately related, Einstein realized that a theory of gravitation, even for static objects, could not involve just the spatial coordinates. The source of the gravitational field, say a material body, must generate a result that effects space-time. Neutron stars are so dense, as we shall soon see, that this becomes an intimate part of their description. It is not possible to formulate Einstein's general theory without getting involved in the considerable apparatus of the geometry of curvilinear coordinates, and measures of curvature such as the Christoffel symbols and the Ricci tensor. This apparatus is most briefly and very clearly given in a small book by P. A. Mi. Dirac based on a course of lectures at Florida State university given in the 1970's. For a complete treatment see the books by Misner, Thorne and Wheeler [1]. and Weinberg [2]. I shall give some small taste of the theory in the appendix. Not unexpectedly, Einstein's field equations can be derived as the conditions for the stationarity of an appropriately defined action. For the gravitational fields the action density involves the metric functions, which describe, in particular how the invariant interval between two space-time events can be measured, $d \tau^{2}=g_{\mu \nu}\left(x^{1}, \cdots x^{4}\right) d x^{\mu} d x^{\nu}$, which in flat space-time is the familiar invariant $d \tau^{2}=d l^{2}-d x^{2}-d y^{2}-d x^{2}$. The gravitational action is (cf. [3]),

$$
\mathcal{L}_{g}=-\frac{1}{16 \pi G} R \sqrt{-g}
$$

where $G$ is Newton's constant, $R$ is the Ricci scalar curvature and $g$ is the determinant of the metric, $g_{\mu \nu}$. We also define $\mathcal{L}_{m}=L_{m} \sqrt{-g}$ for the Lagrangian, $L_{m}$, of the matter fields $\phi$, and construct the total action

$$
I=\int\left(\mathcal{L}_{g}+\mathcal{L}_{m}\right) d^{\mathbf{4}} x
$$

The coupled field equations for the matter and metric functions emerge as the conditions that yield vanishing variation of the action with respect to the metric and matter fields. They can be written as

$$
\frac{\delta \mathcal{L}_{m}}{\delta \phi}-\partial_{\mu} \frac{\delta \mathcal{L}_{m}}{\delta\left(\partial_{\mu} \phi\right)}=0
$$




$$
G^{\mu \nu}=-8 \pi G T^{\mu \nu}
$$

where $G^{\mu \nu} \equiv R_{\mu \nu}-\frac{1}{2} g_{\mu \nu} R$ is Einstein's curvature tensor and $T^{\mu \nu}$ is the matterradiation stress-energy tensor,

$$
T^{\mu \nu} \equiv-g^{\mu \nu} L_{m}+2 \frac{\partial L_{m}}{\partial g_{\mu \nu}}
$$

The first of the field equations are the familiar Euler-Lagrange equations. We shall encounter them in studying theories of dense nuclear matter. The second is Einstein's field equations, and expresses the way in which all forms of energy contributing to the stress-energy warp space-time. The form of the stress-energy tensor appearing above is different from the canonical form familiar in field theory. That they are the same is shown in an example in the appendix.

We don't usually think of the matter Lagrangian and field equations as depending on the metric because we usually ignore gravity and so the metric tensor is a diagonal constant, $g_{\mu \nu}=(1,-1,-1,-1) \delta_{\mu \nu}$. In strong gravitational fields this is not true, and we need to justify it for nuclear and particle shysics as they enter astrophysics. This we now do. In the process we become familiar with gravitational units, and get a qualitative description of neutron stars.

In empty space outside a static spherical star of radjus $R$ and mass $M$, Schwarzschild showed that the solution of Einstein's equations have a simple form. All but the diagonal components of the metric vanish, and they are simple. The line element is

$$
d \tau^{2}=\left(1-\frac{2 G M}{r}\right) d t^{2}-\left(1-\frac{2 G M}{r}\right)^{-1} d r^{2}-r^{2} d \theta^{2}-r^{2} \sin ^{2} \theta d \phi^{2}, \quad(r>R)
$$

A guide to the derivation is given in appendix, which also serves as an introduction to the mathenatics encountered in the general theory. I want to show you that the metric functions, $g_{\mathrm{t}}, g_{\mathrm{rr}}$ in front of $d t^{2}$ and $d r^{2}$ change by an infinitesimal amount over the distance between nucleons in a star that is rear the limit of collapse to a black hole, and hence even for the most massive neutron star. If this is true, and it is as we shall show, it is of great practical importance. It means that we can solve the problem of the matter fields in flat (Minkowski) space. We then put the result of that calculation into the right side of Einstein's equations. This amounts to a partial decoupling. The matter stress-energy (equation of state) can be derived in the absence of gravity and the result put into Einstein's equations to find out how gravity arranges the matter. We will see that a compact star near the limit of collapse has very high density and is what is referred to as a neutron star. To do this I need to show you how to compute in gravitational units $G=1=c$.

$$
\begin{array}{r}
1=c=3 \times 10^{10} \mathrm{~cm} / \mathrm{s} \\
1=G=6.7 \times 10^{-8} \mathrm{~cm}^{3} \mathrm{~g}^{-1} \mathrm{~s}^{-2}
\end{array}
$$


These can be treated as equations so that for example,

$$
\begin{array}{r}
\mathrm{ls}=3 \times 10^{10} \mathrm{~cm} \\
1 \mathrm{~g}=7.4 \times 10^{-29} \mathrm{~cm} \\
1 \mathrm{~s}^{-2}=1.5 \times 10^{7} \mathrm{~g} / \mathrm{cm}^{3} \\
1 \mathrm{erg}=1 \mathrm{~g} \mathrm{~cm}^{2} \mathrm{~s}^{-2}=8.2 \times 10^{-50} \mathrm{~cm}
\end{array}
$$

Next we estimate the mass and radius of a star near the limit. Notice that the metric becomes singular at $r=2 M$. For actual stars, this radius is interior to the star itsclf where the Schwarzschild solution does not hold and the singularity therefore does not exist, but in the special case where the star lies within its "gravitational radius", it must be a black hole. Let us estimate the properties of a star near the limit, $R=2 M$. Assume that gravity packs nurleons up to their hard cores, say $r_{0} \approx 0.5 \times 10^{-13} \mathrm{~cm}$. Then

$$
R \approx r_{0} A^{1 / 3}, \quad M \approx A m
$$

where $A$ is the number of baryons in the star and $m$ is their mass

$$
\begin{aligned}
m=9.10 \mathrm{McV}=1.7 \times 10^{-24} \mathrm{~g} & =1.2 \times 10^{-52} \mathrm{~cm} \\
1 \mathrm{McV} & =1.6 \times 10^{-6} \mathrm{erg}
\end{aligned}
$$

Hence substituting eq.(9) into the equation, $R=2 . M$ we find,

$$
A^{2 / 3}=r_{0} /(2 m)=1.9 \times 10^{38}
$$

Putting this answer back into the expressions for radius and mass we have

$$
\begin{array}{r}
A=2.6 \times 10^{57} \\
R=r_{0} A^{1 / 3}=7 \mathrm{~km} \\
M=R / 2=3.5 \mathrm{~km}=2.3 M_{\odot}
\end{array}
$$

where I used the solar mass,

$$
M_{\odot}=2 \times 10^{33} \mathrm{~g}=1.5 \mathrm{~km}
$$

So here we have an estimate of the baryon number, radius and mass of a star at the limit. He expect a smaller mass and larger radius than the values given by the Schwarzschild relation, because in actual examples where the mutual coupling of gravity and matter is retained [4], instability to collapse sets in even before the Schwarzschild condition is reached, say,

$$
M \approx M_{\odot}, \quad R \approx 10 \mathrm{~km}
$$


The average density of such an object is

$$
\bar{\epsilon}=5 \times 10^{14} \mathrm{~g} / \mathrm{cm}^{3} \approx 2 \epsilon_{0}
$$

where $\epsilon_{0}=2.5 \times 10^{14} \mathrm{~g} / \mathrm{cm}^{3}$ is the energy density of symmetric matter at saturation. Since the density of the star near the limit is supernuclear and since it must be charge neutral else the repulsive Coulomb force will overwhelm gravity, it will be dominated by neutrons and is called a neutron star. Putting the mass and radius into the metric we have (using the fact that at the origin $g_{00}=1$ ),

$$
\frac{g_{r r}(R)}{g_{r r}(0)}=\left(1-\frac{2 M}{R}\right)^{-1}=\left(1-\frac{3}{10}\right)^{-1}=10 / 7
$$

So the metric changes by a small amount over the dimension of the star. It changes by $2 r_{0} / R=2 A^{-1 / 3} \approx 10^{-19}$ of this over the spacing of nucleons in the star. Later we shall also be interested in pulsars with very high angular velocity. Since the gravitational attraction must exceed the centrifugal repulsion else the star would fly apart, we are assured that the curvature of space-time due to rotation must be even less than that due to the mass, which we just saw is completely negligible in any local frame in a region spanning the distance between many nucleons. So in solving the field equations for matter, we make negligible error for neutron stars by solving them in the absence of gravity and then using the resulting stress-energy tensor, which is diagonal in a co-moving frame[2],

$$
T^{\mu, \nu}=\left(\begin{array}{cccc}
\epsilon & 0 & 0 & 0 \\
0 & p & 0 & 0 \\
0 & 0 & p & 0 \\
0 & 0 & 0 & p
\end{array}\right)
$$

in Einstein's field equation to find bow matter is compacted under the influence of gravity.

In the special case of a static star, Einstein's equations take a special form first written down by Oppenheimer and Volkoff. The derivation would take us far afield.

$$
\begin{aligned}
& 4 \pi r^{2} d p(r)= \\
& \quad-\frac{G M(r) d M(r)}{r^{2}}\left(1+\frac{p(r)}{\epsilon(r)}\right)\left(1+\frac{4 \pi r^{3} p(r)}{M(r)}\right)\left(1-\frac{2 G M(r)}{r}\right)^{-1} \\
& d M(r)=4 \pi r^{2} \epsilon(r) d r
\end{aligned}
$$

The interpretation is very simple. Think of a shell of matter in the star of radius $r$ and thickness $d r$. The second equation gives the mass energy in this shell. The pressure of matter exterior to the shell is $p(r)$ and interior to it $p(r)+d p(r)$. The left side of the first equation is the net force acting outward on the surface of the shell by the pressure, and the first factor on the right side is the attractive force 
of gravity acting on the shell by the mass interior to it in Newton's theory. The remaining three factors are the exact corrections for general relativity. So these equations express the balance of internal pressure and gravity. They are the equations of hydrostatic equilibrium in general relativity. The equation of state $p=p(c)$ is the manner in which matter enters the equations of star structure. Otherwise they are completely specified and their correctness is confirmed by the observational tests of general relativity. The most impressive of these are concerned with the pulsar binary known by its celestial coordinates as $1913+16$, and studied for sixteen years [5]. The orbits orientation precesses at a rate of more than 4 degrees per year compared with Mercury, the former classic confirmation which is only 43 seconds per century! Einstein's gravitational radiation prediction, which effects the orbital motion of the binary pulsar pair causing the orbit to decay, is confirmed to less than one percent. All other variants of Einstein's theory of gravitation are ruled out at this level of accuracy. At the rate of decay of the orbit due to the radiation of gravity waves, the two members of the binary will collide in about $10^{8}$ years. While this is a long time, it is to be compared with the galaxy age of $10^{10}$ years. It is of possible importance to the question of strange quark nuggets as cosmic radiation, as we discuss later.

Newton's theory is a special case of the $\mathrm{O}-\mathrm{V}$ equations obtained by dropping the last three factors in eq. 18.

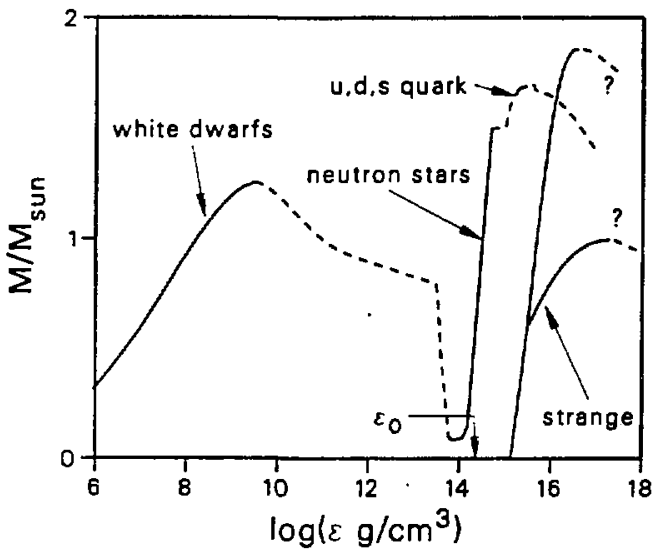

Figure 1: Schematic illustration of the solution of the O-V equations over a broad range of central densities. See discussion in text.

The Oppenheimer-Volkoff equations can be integrated from the origin with the initial conditions that $M(0)=0$ and an arbitrary value for the central energy density $\epsilon(0)$, until the pressure, $p(r)$, becomes zero. That point, $R$, defines the radius of the 
star, and $M(R)$ its mass. For the given equation of state, there is a unique relationship between the mass and central density, $\epsilon(0)$. So for each possible equation of state there is a unique family of stars, parameterized by, say, the central density. For an equation of state spanning a very wide range of densities, a schematic solution is shown in Fig. 1. The abscissa is the central density of the star, which as just discussed can be used to parameterize the unique solution of the Oppenheimer-Volkoff equations for a given equation of state. At central densities about $10^{8}$ the density of say Fe here on earth, there is the famous class of stars called white dwarfs. They consist of highly compressed very neutron rich iron and nickel isotopes. As their mass approaches $1.4 M_{\odot}$ they become unstable: They are supported against gravitational collapse by the pressure of relativistic electrons. For high enough mass, this is insufficient, and the star becomes unstable. Though very dense by earthly standards, white dwarfs are five orders of magnitude less dense than neutron stars, which range roughly in central density from a little below nuclear density to 5 or 10 times above $\left(\sim 10^{14} \times\right.$ the density of iron). It is the Fermi pressure of the nucleons and eventually for high densities, the repulsive nuclear force that stabilizes neutron stars. It should be clear, but is worth mentioning in any case, that neutron stars are held together by the gravitational attraction and not by the nuclear force. After all nuclei themselves are stable only for $A<250$ and neutron matter not at all. Indeed, except possibly for the very lightest neutron stars, gravity has compacted the neutron matter so severely that the most important part of the nuclear force is the repulsion. If gravity were switched off a neutron star would explode! The pressure in the core of the more massive neutron stars may be so high that baryons dissolve into a quark matter phase. At typical densities of the core it is easy to confirm that the chemical potential of two flavor (non-strange) quark matter will exceed the strange quark mass, so that if there is a quark core, it will contain all three flavors of quarks. We also show in Fig. 1 an hypothetical family of strange stars. These are pure quark stars, and could exist if strange quark matter is lower in energy per nucleon number than the mass of the nucleon. As we will discuss later in detail, this hypothesis is not only plausible, but is not ruled out by any known fact.

Of course we do not know the equation of state of dense nuclear or neutron star matter. Therefore in Fig. 6 several families are sliown for different values of the nuclear compression. It will be noted that each family has a maximum mass star, called the limiting mass and that the central density of the limiting mass star is higher the softer ${ }^{1}$ the equation of state. The part of the curve for which the slope is positive corresponds to stable configurations. For negative slope, one can readily verify that the star is unstable to radial perturbations. In fact those beyond the maximum are unstable to collapse to black holes or to dispersal, depending on the sense of any perturbation that moves them off their equilibrium configuration.

${ }^{1}$ When two equations of state in the form $p=p(\epsilon)$ vs $\epsilon$ or $c(\rho) / \rho$ vs $\rho$, where $p, \epsilon, \rho$ are pressure, energy density and baryon density, the one for which $p$ or $c / \rho$ is smaller at the same value of the independent variable is said to be softer, the other stiffer. 
It is in the limiting mass that a constraint on the equation of state arises. Obviously an acceptable equation of state must have a limiting mass at least as large as the largest observed mass. It is of some importance to have reliable estimates of the maximum neutron star mass, so that a distinction can be made between light black holes whose only signature would be their gravitational effect on a companion, from a neutron star that is otherwise also invisible, whether because it is not producing pulsed radiation, or because we fo not happen to lie on the cone swept out by the radiation during the stars rotation.

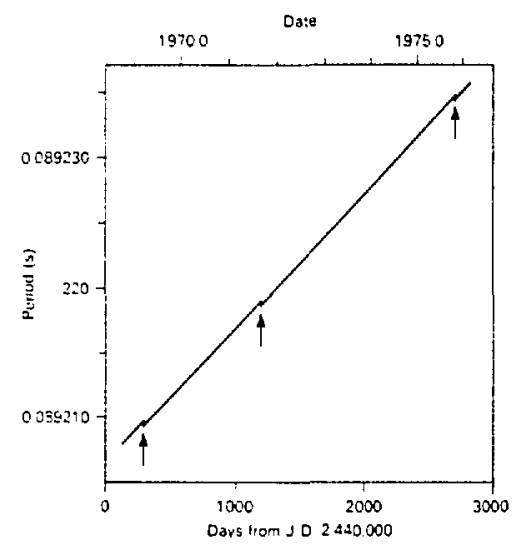

Figure 2: Showing the slow variation of the period of the Vela pulsar, $\dot{P}=1.2503 \times 10^{-13} \mathrm{~s} / \mathrm{s}$. Also three "glitches" are seen in the time frame, thought to correspond to star quakes. [6]

\section{Why Pulsars are Neutron Stars}

A bout 400 pulsars have been found since the discovery by Hewish and Bell in 1967 of the pulsed signals received by a radio-telescope. The period of the pulses range from milliseconds to seconds, and is interpreted as the period of a rotation. Why? Since the period is observed on earth using a radio telescope we know that the pulsar is losing energy. Over time the amplitude of vibrations is diminished by energy loss, but not the frequency. For rotation the frequency is damped. This is what is observed, as in the example shown in Fig. 2. Although by most normal standards the period of pulsars is very stable, it does measurably change over time, and depending on the period, its rate of change can be determined by making observations over time of days to weeks or months. Known pulsars have period rate of changes falling in the range,

$$
10^{-18}<\dot{P}<10^{-13} \mathrm{~s} / \mathrm{s}
$$

In addition, ordinary stars have magnetic fields ( $\sim 100$ gauss) and rotate. When they collapse from a radius of $10^{6} \mathrm{~km}$ to $10 \mathrm{~km}$, both the rotation frequency and field are 
scaled up by the conservation laws of angular momentum and magnetic flux. The field is typically scaled to $10^{12}$ gauss. There is other evidence of such strong fields. The remnant of the crab supernova is still accelerating with an apparent energy input of $\sim 10^{38} \mathrm{ergs} / \mathrm{s}$, and the most likely source of input energy is the absorption of magnetic dipole radiation from the fast pulsar within it. Energy balance implies about the same strength for the magnetic field as quoted above,

$$
10^{38} \mathrm{ergs} / \mathrm{s}=-\frac{d E}{d t}=-\frac{d}{d t}\left(\frac{1}{2} I \omega^{2}\right)=\frac{1}{6} R^{6} B^{2} \omega^{4} \sin ^{2} \alpha
$$

Given the observed period and rate of change of period,

$$
P \sim \frac{1}{30} \mathrm{~s}, \quad \dot{P} \sim 4 \times 10^{-13} \mathrm{~s} / \mathrm{s}
$$

we find (taking $\sin \alpha=1$ ),

$$
\begin{aligned}
B \sim 4 \times 10^{12} \text { gauss, } & I \sim 2 \times 10^{4.4} \mathrm{~g} \mathrm{~cm}^{2} \\
E_{\text {rot }} & \sim \frac{1}{2} I \omega^{2} \sim 4 \times 10^{48} \mathrm{ergs}
\end{aligned}
$$

where I use

$$
3.5 \times 10^{24} \text { gauss } \mathrm{cm}=1
$$

in gravitational units. The field will in general be oriented in a different direction than the rotation axis, say by an angle $\alpha$. It is believed, but not understood, that radiation over a broad band of frequencies is emitted within some angular spread along the magnetic axis $[\overline{7}, 8]$. Given the rotation, one has a beacon which we see as pulses as the star rotates.

We can use the period of rotation to estimate an average energy density of a millisecond pulsar. For the star to hold together under the opposing forces of gravity and centrifuge, we must have,

$$
\alpha^{2} \frac{G m M}{R^{2}}>m \Omega^{2} R
$$

Hence for the average density,

$$
\bar{\epsilon}=M /\left(\frac{4 \pi}{3} R^{3}\right)>\frac{3 \pi}{(\alpha P)^{2}}=3.3 \times 10^{14} \mathrm{~g} / \mathrm{cm}^{3}
$$

where the last equality holds for a millisecond pulsar. The factor $\alpha$ is unity in Newtonian physics and is empirically found to be about 0.65 in General relativity [9]. Since $\epsilon_{0} \approx 2.5 \times 10^{14} \mathrm{~g} / \mathrm{cm}^{3}$, we learn that the average density of a pulsar has to be about as large or larger than nuclear density and se pulsars must be neutron stars. In actual models that I have studied, $\bar{\rho} / \rho_{0}$ ranges from 3 for stiff equations of state to 5 for 
the standard compression found a decade ago in the analysis of the giant monopole resunance in nuclei $(K=210 \mathrm{MeV})$ to 6 or more for softer equations of state. Clearly the stiffer the equation of state the more sensitive the neutron star limiting mass will be to properties near saturation.

The range of densities in neutron stars is enormous, ranging from matter at $\sim 10^{15}$ times that of ordinary metals here on earth to zero, the last millimeter of gas in the atmosphere. The density profiles of neutron stars ranging from one at the limiting mass of the equation of state from which it is constructed, to a rather light one, are shown in Fig. 3. Notice that the central region is in all cases highly compacted, and fairly constant, and the edre is very abrupt, except for the lightest which has a very thick crust. The kinks and wiggles are not numerical inaccuracies but correspond to features of the equation of state. Threc regions, and the source for and general nature of the equation of state in each is indicated in Table 1.

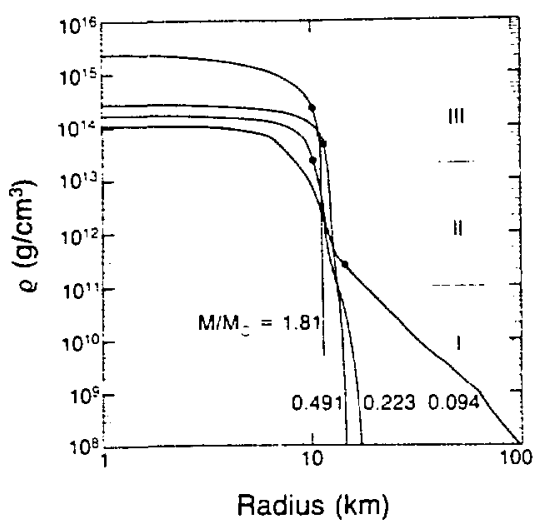

Figure 3: Energy density profile of several neutron stars. Dots show point interior to which $95 \%$ of the stars mass is contained. Roman numerals refer to threc density ranges of equation of state ( see table 1). [10]

\section{Chemical Potentials and Phase Equilibria}

Neutron stars are not made purely of neutrons as their name suggests, for such a star is beta unstable. They are instead a mixture of many species that is in generalized beta stability or said otherwise, in equilibrium, under the constraint of charge neutrality. A star is charge neutral to a high degrec because gravity is the force that holds it together, and gravity is much weaker than the repulsive Coulomb force so that all but a very small excess of net charge will be expelled from the star. So w must learn 
Table 1: Density regions needed to describe neutron star surface, I, II, and interior, III.

\begin{tabular}{c|ccc} 
Region & $\begin{array}{c}\text { Density } \\
\mathrm{g} / \mathrm{cm}^{3}\end{array}$ & $\begin{array}{c}\text { Nature of } \\
\text { Matter }\end{array}$ & Reference \\
\hline I & $2 \times 10^{3}<\rho<1 \times 10^{11}$ & $\begin{array}{c}\text { crystalline: } \\
\text { light metals }\end{array}$ & {$[11]$} \\
\hline II & $1 \times 10^{11}<\rho<2 \times 10^{13}$ & $\begin{array}{c}\text { crystalline } \\
\text { heavy metals, } \\
\text { relativistic electrons }\end{array}$ & {$[12]$} \\
\hline III & \multicolumn{3}{r}{$\begin{array}{c}\text { relativisitic } \\
\text { nucleons, hyperons, } \\
\text { leptons (pions) }\end{array}$} \\
\hline
\end{tabular}

how to construct equilibrium mixtures of particles. Secondly, at high density, perhaps realized in the cores of neutron stars, we believe that a phase transition from the confined state of individual hadrons to the unconfined state of quark matter occurs. In the latter case the quarks are confined over a much larger region than the space occupied by a single nucleon. and in the astronomical context, over a macroscopic region. Within this region. they are relatively free. We are therefore also interested in the pussible transition between phases.

\subsection{Chemical Equilibrium}

Chemical potentials are used to express the condition of equilibrium of a multicomponent system in which transformations among the components are possible. The transformations define relations among the chemical potentials. Usually there are conservation laws involving attributes of the particles, such as baryon number and electric charge. Then the chemical potentials of all components can be written in terms of as many independent chemical potentials as there are such conservation laws. Finally the independent chemical potentials are determined by the conservation laws themselves. It is important to realize that the equilibrium chemical composition can be found without any detailed knowledge of reaction rates, nor indeed is it necessary to enumerate any more of the transformations or reactions among the constituents as is required to write their chemical potentials in terms of those representing the conserved attributes. One example of a conservation law or constraint is that of charge neutrality of a star. The reason for the constraint is that the repulsive Coulomb force is so much stronger than the gravitational, that a star can tolerate very little net charge. Above a very small limit that we will calculate later, excess charged particles of the same sign will simply be expelled. Thus neutron star matter is to high accuracy charge neutral. The other independent chemical potential in this example is that for 
baryon number. It can be determined by the density at which we want to obtain the energy and pressure of the matter.

From the quark content of the baryons it is easy to establish, using table 2, the connection between the quark chemical potentials and those for baryon number (as for the neutron), electric charge and strangeness,

$$
\begin{gathered}
\mu_{b}=\mu_{u}+2 \mu_{d} \\
\mu_{q}=\mu_{u}-\mu_{d} \\
\mu_{k}=\mu_{d}-\mu_{s}
\end{gathered}
$$

Here, $\mu_{b}, \mu_{q}, \mu_{k}$ denote the chemical potentials for positive unit baryon number, electric charge and strangeness respectively. (The $k$ stands for $k^{0}$ in this context.) For any system in which these are the conserved particle attributes, the chemical poteritial of any particle can be expressed as,

$$
\mu_{1}=b_{i} \mu_{b}+q_{i} \mu_{q}+s_{i} \mu_{k}
$$

where $b_{1}, q_{i}, s_{i}$ arc the baryon number, electric charge and strangeness of the particle $i$, all in integer units. The above relations are usually needed in computing the phase equilibrium and transition point between hadronic and quark-gluon phase.

\subsection{Phase Equilibrium}

Consider the following schematic problem for a neutron star which is assumed to consist of neutrons, protons and electrons at low and moderate density; but which in the interior has such a high density that the baryons are converted to quark matter. First we write the relation that connects the hadron chemical potentials using the transformation $n \leftrightarrow p+\epsilon+\bar{\nu}_{\varepsilon}$. Both directions on the arrow indicate that we are interested in the equilibrium condition. Neutrinos leak out of the star because, being massless. they have the escape velocity so that their number is not conserved. Therefore we set $\mu_{\nu}=0$. From this the proton chemical potential can be written in terms of the neutron (for baryon charge) and electron (for negative electric charge) chemical potentials, $\mu_{p}=\mu_{n}-\mu_{e}$. This is a simple example of a general fact: Upon writing down the transformations (reactions) among the species in a chemical equilibrium, chemical potentials of all species can be written in terms of just the number of chemical potentials that correspond to conserved charges. In this case, baryon and negative electric charge. In a star we require charge neutrality, the two long-range forces in question being such that any' excess net charge would be expelled by the repulsive Coulomb force. The charge can be written in terms of the chemical potentials analogous to eq. (39) below, since the total charge density will involve integrals over the filled Fermi seas of charged particles, and the chemical potentials will appear either in momentum distribution functions or in limits of integration. Charge neutrality,

$$
q_{H}\left(\mu_{n}, \mu_{p} \cdot \mu_{e}, T\right)=0 .
$$


provides a second relation among the chemical potentials, where $q_{H}$ denotes the electric charge density in the hadronic phase. So there is one independent chemical potential, say $\mu_{\mathrm{n}}$ which we now write $\mu \equiv \mu_{n}$ for brevity. So

$$
\mu_{p}=\mu_{p}(\mu), \quad \mu_{e}=\mu_{e}(\mu) .
$$

Assume at this point that when the density of neutron star matter as described here is high enough, it will undergo a phase transition to a quark-gluon plasma, which we assume here to consist of an equilibrium mixture of $u, d$ quarks and electrons (for charge neutrality). So we can write the $\mu_{u}, \mu_{d}$ in terms of $\mu_{n}(=\mu), \mu_{e}$ using eq. (27). Our first problem then is to find the point at which the hadron phase begins to turn into the quark phase. This is the beginning of the mixed phase. At its boundary as approached from the hadron phase, we must have the temperatures, pressures and chemical potentials of the two phases equal. This assures thermal, mechanical and chemical equilibrium respectively. There is an expression for the pressure in each phase as a function of chemical potentials of the constituents of that phase, and the temperature. An example in the quark phase is seen in eq. (47) below. Such an expression containing the quark chemical potentials can be rewritten in terms of the hadron and electron chemical potentjals using eq. (27), to give an expression for the pressure in the quark phase, $p_{Q}\left(\mu, \mu_{p}, \mu_{e}, T\right)$. In the hadronic phase and at the boundary between hadron and mixed phase the relation eq. (30) holds so we have at the boundary,

$$
p_{H}\left(\mu, \mu_{p}(\mu), \mu_{e}(\mu), T\right)=p_{Q}\left(\mu, \mu_{p}(\mu), \mu_{e}(\mu), T\right)
$$

So the above is an equation that $\mu$ satisfies on the boundary between the hadron and mixed phases. We could sketch a plot of $p$ versus $\mu$ for each phase. Because of the $-B$ in the pressure expression, eq (37), in the quark phase, at low $\mu$ it will lie below the pressure in the hadron phase. So for $\mu$ below the crossing point, which is the solution to the above equation, matter will exist in the hadron phase. The solution to the above equation defines the location of one point in the $T-\mu$ plane. Solving it at many values of $T$ defines the boundary between hadron and mixed phases.

Similarly, in the quark phase charge neutrality,

$$
q_{Q}\left(\mu_{n}, \mu_{p}, \mu_{e}\right)=0,
$$

defines a relation between the chemical potentials, but a different one than in the hadron fhase. Let its solution be expressed as

$$
\mu_{p}=\mu_{p}^{\prime}(\mu), \quad \mu_{e}=\mu_{e}^{\prime}(\mu)
$$

We get a different equation for $\mu$ on the boundary between the mixed phase and quark phase, and hence a different boundary than described above. It is determined as the solution of,

$$
p_{H}\left(\mu, \mu_{p}^{\prime}(\mu), \mu_{e}^{\prime}(\mu), T\right)=p_{Q}\left(\mu, \mu_{p}^{\prime}(\mu), \mu_{e}^{\prime}(\mu), T\right)
$$


The pressure also will in general be different on this boundary at the same $T$, and all the chemical potentials as well, as compared to the other boundary. In the mixed phase all these quantities will vary continuously from the hadron boundary to the quark boundary. In an entirely analogous way to that described above, the boundary in the $T-\mu$ plane between the mixed and quark phase can be drawn.

In the mixed phase between the above described boundaries the expression for charge neutrality involves the charge of both participating phases,

$$
(1-\chi) q_{H}\left(\mu_{n}, \mu_{p}, \mu_{e}, T\right)+\chi q_{Q}\left(\mu_{n}, \mu_{p}, \mu_{e}, T\right)=0
$$

where $X=\left(\rho-\rho_{H}\right) /\left(\rho_{Q}-\rho_{H}\right)$ (and $\rho_{H}, \rho_{Q}$ denote the baryon number density in the hadron and quark phases at the boundary between hadron and mixed phase and mixed phase and quark phase respectively) varies from 0 to 1 at the hadron and quark boundary respectively, and denotes the volume ratio of quark to hadron phase in the mixture. As before this equation allows us to solve for $\mu_{p}, \mu_{e}$ as functions of $\mu$, which varies between its two boundary values. Then all other quantities of interest, which are the same linear combination of hadron and quark quantities in the mixed phase as above, can be computed.

We have outlined how the chemical potentials can be derived in terms of $T, \mu$ in the entire plane. So we can calculate all quantities depending on them, such as the energy density, baryon density, etc. The above problem may seem more involved than the usual text book problem (cf. Landau and Lifshitz). That is because here we have two conserved quantities, baryon and electric charge, for which there are independent chemical potentials, in terms of which all others can be written. In the usual examples (gas-liquid) there is only one. In the latter case, the phase coexistence region is a line in the $T-\mu$ plane. In our case it is a region bounded by two lines, which owe their existence in our example to the fact that there are two (or more) conservation laws. (For an example of three conservation laws, appropriate to baryon, charge and strangeness conservation see [13]).

Frequently, instead of the baryon chemical potential as independent variable, one would like to have the baryon number density. This one can of course obtain in the three regions by employing the expression of the baryon number as a function of chemical potentials.

\section{Quark Matter}

We do not have a rigorous theory of dense nuclear matter and QCD is not a theory for which we can derive practical results for the equation of state either. We shall begin therefore with a simple model of quark matter, based on the notion of asymptotic freedom and the MIT bag model. This is actually easier, because it is crude, than the more difficult problem of hadronic matter in the confined phase, that we take up later. 
We assume that the quarks are free within the spatial region occupied by quark matter, and that this region differs by a volume energy from the ordinary vacuum of confined quarks (hadronic matter). We can get analytical expressions in two limits,

$$
\begin{array}{ll}
\text { 1) } T=0, m \neq 0 & \text { 2) } T \neq 0, m=0
\end{array}
$$

from the expressions for a Fermi gas of quarks. The pressure, energy density, baryon number density and entropy density are

$$
\begin{aligned}
p & =\sum_{f} \frac{1}{3} \frac{\gamma_{f}}{2 \pi^{2}} \int_{0}^{\infty} \frac{k^{2}}{\epsilon_{f}(k)}\left(n\left(k, \mu_{f}\right)+n\left(k,-\mu_{f}\right)\right) k^{2} d k-B \\
\epsilon & =\sum_{f} \frac{\gamma_{f}}{2 \pi^{2}} \int_{0}^{\infty} \epsilon_{f}(k)\left(n\left(k, \mu_{f}\right)+n\left(k,-\mu_{f}\right)\right) k^{2} d k+B \\
\rho & =\sum_{f} \frac{1}{3} \frac{\gamma_{f}}{2 \pi^{2}} \int_{0}^{\infty}\left(n\left(k, \mu_{f}\right)-n\left(k,-\mu_{f}\right)\right) k^{2} d k \\
s & =\frac{S}{V}=\left(\frac{\partial p}{\partial T}\right)_{V, \mu \rho}
\end{aligned}
$$

where $\epsilon_{f}(k)=\sqrt{m_{f}^{2}+k^{2}}$ and

$$
n\left(k, \mu_{S}\right)=\left(\exp \left[\left(\epsilon_{S}(k)-\mu_{S}\right) / T\right]+1\right)^{-1}
$$

is the Fermi distribution function. The factor $1 / 3$ occurs in the baryon density because there are three quarks per baryon. The bag pressure is denoted by $B$, and represents the positive energy shift per unit volume $i_{n}$ the deconfined vacuum relative to the confined vacuum. The quark degeneracy for each flavor is $\gamma_{f}=2_{\text {spin }} \times 3_{\text {color }}$.

Other properties of possible interest are for example the electric charge density or the strangeness,

$$
q=\sum_{f} \frac{\gamma_{f}}{2 \pi^{2}} q_{f} \int_{0}^{\infty}\left(n\left(k, \mu_{f}\right)-n\left(k,-\mu_{f}\right)\right) k^{2} d k
$$

where $q_{f}$ is the quark electric charge.

\subsection{Zero Temperature}

For the first of the two limiting cases above, the distribution functions become step functions at the energy $\mu_{f}$. In that case we can use standard integrals (ref. [14]. p87), to express the results. For example,

$$
\begin{aligned}
& \int_{0}^{k} \frac{k^{4}}{\sqrt{m^{2}+k^{2}}} d k \\
& =\frac{1}{4}\left[k^{3} \sqrt{k^{2}+m^{2}}-\frac{3}{2} m^{2} k \sqrt{m^{2}+k^{2}}+\frac{3}{2} m^{4} \ln \left(\frac{\sqrt{k^{2}+m^{2}}+k}{m}\right)\right]
\end{aligned}
$$


Table 2: The six known quarks and their quantum numbers

\begin{tabular}{c|cccccccc} 
Name & $\begin{array}{c}\text { Mass } \\
(\mathrm{MeV})\end{array}$ & $\begin{array}{c}\text { baryon } \\
\text { number }\end{array}$ & $\begin{array}{c}\text { charge } \\
\text { isospin } \\
\text { projection }\end{array}$ & $\begin{array}{c}\text { strange- } \\
\text { ness }\end{array}$ & charm & beauty & truth \\
\hline $\mathbf{u}$ & $\mathbf{5}$ & $1 / 3$ & $2 / 3$ & $1 / 2$ & 0 & 0 & 0 & 0 \\
$\mathrm{~d}$ & 7 & $1 / 3$ & $-1 / 3$ & $-1 / 2$ & 0 & 0 & 0 & 0 \\
$\mathrm{~s}$ & 150 & $1 / 3$ & $-1 / 3$ & 0 & -1 & 0 & 0 & 0 \\
$\mathrm{c}$ & 1500 & $1 / 3$ & $2 / 3$ & 0 & 0 & 1 & 0 & 0 \\
$\mathrm{~b}$ & 5000 & $1 / 3$ & $-1 / 3$ & 0 & 0 & 0 & 1 & 0 \\
$\mathrm{t}$ & $>80000$ & $1 / 3$ & $2 / 3$ & 0 & 0 & 0 & 0 & 1 \\
\hline
\end{tabular}

We find

$$
\begin{aligned}
p & =-B+\sum_{f} \frac{1}{4 \pi^{2}}\left[\mu_{f}\left(\mu_{f}^{2}-m_{f}^{2}\right)^{1 / 2}\left(\mu_{f}^{2}-\frac{5}{2} m_{f}^{2}\right)+\frac{3}{2} m_{f}^{4} \ln \left(\frac{\mu_{f}+\left(\mu_{f}^{2}-m_{f}^{2}\right)^{1 / 2}}{m_{f}}\right)\right] \\
\epsilon & =B+\sum_{f} \frac{3}{4 \pi^{2}}\left[\mu_{f}\left(\mu_{f}^{2}-m_{f}^{2}\right)^{1 / 2}\left(\mu_{f}^{2}-\frac{1}{2} m_{f}^{2}\right)-\frac{1}{2} m_{f}^{4} \ln \left(\frac{\mu_{f}+\left(\mu_{f}^{2}-m_{f}^{2}\right)^{1 / 2}}{m_{f}}\right)\right] \\
\rho & =\sum_{f} \frac{\left(\mu_{f}^{2}-m_{f}^{2}\right)^{3 / 2}}{3 \pi^{2}}
\end{aligned}
$$

where $k_{F j}$ is the Fermi momentum defined in ierms of the chemical potential, $\mu_{f}=$ $\left(m_{f}^{2}+k_{F f}^{2}\right)^{1 / 2}$. The above results are suitable for compact star calculations in which the nucleons are believed to be dissolved into quarks by the high pressure in the interior of the star, since temperatures are in the $\mathrm{KeV}$ region for compact stars shortly after birth and therefore completely negligible on the nuclear scale.

\subsection{Massiess Quark Approximation}

We can also obtain analytic expressions if the quarks are massless, even if the temperature is finite. After some manipulation and using the standard integrals,

$$
\int_{0}^{\infty} d x \frac{x}{1+e^{x}}=\frac{\pi^{2}}{12}, \quad \int_{0}^{\infty} d x \frac{x^{3}}{1+e^{x}}=\frac{7}{120} \pi^{4}
$$

we can obtain the explicit forms:

$$
\begin{aligned}
p & =\sum_{f}\left(\frac{7}{60} \pi^{-} T^{4}+\frac{1}{2} T^{2} \mu_{f}^{2}+\frac{1}{4 \pi^{2}} \mu_{f}^{4}\right)-B \\
\epsilon & =3 p+4 B
\end{aligned}
$$




$$
\begin{aligned}
& \rho=\sum_{f} \frac{1}{3}\left(T^{2} \mu_{f}+\frac{\mu_{f}^{3}}{\pi^{2}}\right) \\
& s=\sum_{\rho}\left(\frac{7}{15} \pi^{2} T^{3}+T \mu_{f}^{2}\right)
\end{aligned}
$$

For the massiess Bose gas of gluons of degeneracy $\gamma_{g}=2 \times 8_{c}$, we similarly obtain the contributions;

$$
\begin{aligned}
p & =\frac{8 \pi^{2}}{45} T^{4} \\
\epsilon & =3 p \\
s & =\frac{32 \pi^{2}}{45} T^{3}
\end{aligned}
$$

where we use,

$$
\int_{0}^{\infty} \frac{x^{3} d x}{e^{x}-1}=\frac{\pi^{4}}{15}
$$

For massless quarks and gluons, we thus have, for example,

$$
p=\frac{8 \pi^{2}}{45} T^{4}+\sum_{f}\left(\frac{7}{60} \pi^{2} T^{4}+\frac{1}{2} T^{2} \mu_{f}^{2}+\frac{1}{4 \pi^{2}} \mu_{f}^{4}\right)-B
$$

The lowest order gluon interactions have been calculated $[15.16,17]$, and result in the following modification,

$$
\begin{aligned}
p= & \frac{8 \pi^{2}}{45} T^{4}\left(1-\frac{15 \alpha_{s}}{4 \pi}\right) \\
& +\sum_{f}\left(\frac{7}{60} \pi^{2} T^{4}\left(1-\frac{50 \alpha_{s}}{21 \pi}\right)+\left(\frac{1}{2} T^{2} \mu_{f}^{2}+\frac{1}{4 \pi^{2}} \mu_{j}^{4}\right)\left(1-\frac{2 \alpha_{s}}{\pi}\right)\right)-B
\end{aligned}
$$

and corresponding changes in $\epsilon, s=\partial p / \partial T$ and $\rho=\frac{1}{3} \sum_{f} \partial p / \partial \mu_{f}$. The coupling constant is denoted by $\alpha_{s}$. (Many authors have employed a common chemical potential for all flavors and apply a factor $N_{f}$ to the quark contributions to the $\epsilon, p$ etc. This is incorrect for $N_{f}=3$, since such matter is neither charge symmetric nor has it zero strangeness.)

\subsection{Quark Star Matter}

In the preceding subsections we have derived expressions for the equation of state of quark matter in the bag model. These will be useful to us later in connection with the possible existence of neutron stars with quark matter cores. Let us in this section consider, as an example, hypothetical stars made of quark matter. We will discuss this topic at a more advanced level in the last section. 
One thing that we have remarked about stars is that they are charge neutral, and that they are equilibrium configurations; that is to say they are a mixture of species that are in chemical equilibrium at their temperature (effectively zero for a mature neutron star) and therefore at their lowest energy. Sirce the time scale of a star is effectively infinite compared to the weak interaction time scale, and weak interactions violate strangeness conservation, then quite obviously the strangeness quantum number of quarks is not conserved in a star. Any neutrinos or photons that are produced in the decay of a high momentum $u$ or $d$ quark near the top of the Fermi surface, to a low momentum s quark will leak ou: of the star. The star's energy is thereby lowered by loss of their energy and by lowering of the average energy of the quarks. Chemical equilibrium is therefore found without regard to the conservation of strangeness of the quarks.

We have derived expressions for the pressure and energy density of quark matter in terms of the quark chemical potentials above. Now we will need to discuss how these latter quantities are determined. According to the above discussion of strangeness non-conservation, the relationships between the quark chemical potentials are determined solely by conservation of electric charge and baryon charge conservation. So possible transformations that conserve these charges can be written with reference to table 2 .

$$
\begin{array}{r}
\mu_{d}=\mu_{s} \\
\mu_{d}=\mu_{u}+\mu_{e} \\
\mu_{c}=\mu_{u}
\end{array}
$$

Note that these follow also from eq. (27) with $\mu_{k}=0$ (for strangeness non-ronservation) and $\mu_{e}=-\mu_{q}$. From an expression for electric clarge density we can write the condition of charge neutrality. From eq. (41) we can write,

$$
q=\sum_{f}\left\{q_{f} \frac{\left(\mu_{f}^{2}-m_{f}^{2}\right)^{3 / 2}}{\pi^{2}}\right\}-\frac{\left(\mu_{e}^{2}-m_{e}^{2}\right)^{3 / 2}}{3 \pi^{2}}=0
$$

where the last term is the electron contribution. Finally the expression for the baryon number density in eq. (41) provides a fifth relation for chosen density with which the five chemical potentials $\mu_{u}, \mu_{d}, \mu_{s}, \mu_{c}, \mu_{e}$ can be determined.

Knowing the quark chemical potentials we can now compute the pressure and energy density of the quarks. Since the electron mass is small it can be neglected, or kept in analogous expressions to eq. (41) for electron contribution. The $B$ should be dropped, and the electron statistical weight, 2, substituted for quark weight, 6 ; ie multiply $p, \epsilon$ by $1 / 3$. The result is then added to the pressure and energy of the quarks to get the total. This provides a complete description in the approximation of the bag model to the equation of state for quark star matter. 


\subsection{Strange and Charm Stars}

As a hypothetical example let us use the equation of state described just now for cold quark star matter to compute the structure of stars made from it. This is done by solving eqs. $(18,19)$, with the above equation of state for the pressure and energy density. At low and moderate baryon densities, which we can estimate from eq. (41) (putting $m_{f}=0$ for ease), we find that the chemical potential for charm does not approach the magnitude of the charm quark mass, and so it is not populated, while the strange quark is populated at moderate density. However at very high density the charm quark is also present. We choose a value of the bag constant that yields an energy per baryon at $p=0$ of $E / A=c / \rho=990 \mathrm{MeV}$; ie above the nucleon mass. In Fig. 4 the resulting star mass as a function of central density is shown. The lower curve is the gravitational mass of the star, defined in eq. (19); ie

$$
M=4 \pi \int_{0}^{R} \epsilon(r) r^{2} d r
$$

The upper curve is the mass of the equivalent number of baryons if the star were disassembled into neutrons at infinity. It is obtained by integrating the number density over the proper volume, which can be read from the Schwarzschild metric as $d V=\epsilon^{\lambda} r^{2} d r d \cos \theta d \phi$.

$$
M_{A}=4 \pi m_{n} \int_{0}^{R} \rho(r) /\left(1-\frac{2 G M(r)}{r}\right)^{1 / 2} r^{2} d r
$$

where $m_{n}$ is the neutron mass. (We have not proven it, but it is true that the spatial metric has the same form inside and outside the star.

On the gravitational mass curve the regions of positive slope are stable according to the qualitative argument given in the appendix. Closer study[11] shows that in the absence of viscosity the sequence of stars at high density, $\epsilon_{c} \sim 10^{18} \mathrm{~g} / \mathrm{cm}^{3}$, in the neutron star models studied in the early 1960's, are unstable to long wavelength radial vibrations which would carry some of the very high density matter in the core inside its Schwarzschild radius, and thus lead to collapse of the star to a black hole. It is not clear at this time whether viscosity would stabilize the star, as it does in the case of rotation. What is true is that the bulk viscosity of quark matter is very high[18]. Stability to vibrational modes is a difficult subject and is beyond the scope of these lectures. What is true is that such stars are stable against disassembly of their equivalent number of neutrons to infinity, because the gravitational mass lies always below the baryon mass $\left(M_{A}\right)$. This is in contrast to the analogous high density family of neutron stars studied in the 1960's which where therefore manifestly unstable configurations[19].

In case viscosity does stabilize the high density stars, they would constitute a new family of stars, which we could call charm stars because of the presence of charm quarks in their cores. The quark distribution of such a star near the peak in mass is shown in fig. 5 . 


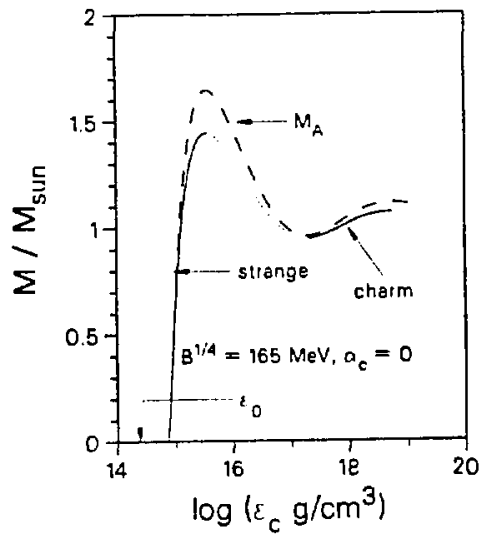

Figure 4: Gravitational mass of strange and charm stars compared to mass at infinity, $M_{A}$, of the equivelent number of neutrons as correspond to quark number, in the case that strange quark matter has equilibrium energy per nucleon $50 \mathrm{MeV}$ above the nucleon mass.

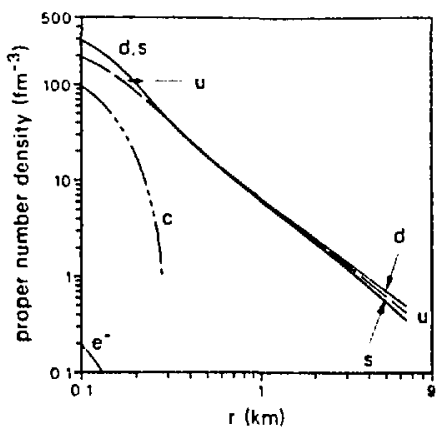

Figure 5: The distribution of quarks in a charm star near the limiting mass of the family.

How could such stars be made? Almost certainly not in a supernova. Either neutron stars or strange stars are of lower density and are the ground state, and would be the natural product, depending whether hadronic or strange matter is absolutely stable, respectively. They could be relic stars from the early universe, having formed while the density of matter was still very high. This scenario needs to be investigated in detail. Otherwise it is conceivable that they are made in the relativistic collision of neutron (or strange) stars. The collision energy would serve to compress the matter, the outer lying layers forming the inertial confinement within which the compression is achieved.

Notwithstanding the uncertain creation mechanism, it is interesting that another long lived compact star configuration may exist, subject of course to verification of stabilization of vibrational modes by viscosity, within a wide range of equilibrium values of $E / A$ for quark matter from significantly above the nucleon mass to anything below. We recall that on theoretical grounds we expect the energy per nucleon of strange matter to be near (within 10's of $\mathrm{MeV}$ ) that of the nucleon itself[20, 21]. 


\section{Nuclear and Neutron Star Matter}

The idealized matter of the interior of nuclei and the matter of neutron stars have similarities and differences which need to be understood. The similarities include the fact that they are composed of hadrons and the densities are the same within an order of magnitude or less. The differences arise from two facts. (1) Nuclei are bound by the charge symmetric nuclear force, but neutron stars are bound by gravity. Hence in nuclei, $N \approx Z$. However since the repulsive Coulomb force is so much stronger than the gravitational force that binds stars, the net charge in a star must be very small,

$$
\frac{\left(Z_{\text {net }} e\right) \epsilon}{R^{2}}<\frac{G(A m) m}{R^{2}} \longrightarrow Z_{\text {net }}=Z_{p}+Z_{e}<10^{-36} A
$$

(where we use $\hbar c / e^{2}=137$ and $\hbar=1.1 \times 10^{-27} \mathrm{~g} \mathrm{~cm}^{2} \mathrm{~s}^{-1}=2.6 \times 10^{-66} \mathrm{~cm}^{2}$.) Although $Z_{p}$ is not nearly so small as $Z_{\text {net }}$, it is considerably smaller than $A / 2$. So nuclei are symmetric and neutron stars are asymmetric. (2) There is another and profound difference arising from the weak interaction time scraie $\tau_{w} \sim 10^{-10}$ seconds. Because of the high density of neutron stars and the fact that baryons obey the Pauli principle, it is energetically favorable for nucleons at the $t$ p of the Fermi sea to convert to other baryons, including strange ones (hyperons). 'ithis is possible because strangeness is conserved only on the strong interaction time scale, not on the weak. Even the time scale of supernova is long compared to that of the weak. So strangeness is not conserved in astrophysical objects. It wouldn't be conserved in stable nuclei either, but energetically it is not favorable to have hyperons in the ground state because their masses exceed the nucleon mass by more than the Fermi energy of the nucleons. Nuclear reactions on the other hand are so fast that strangeness is conserved. So the matter studied in nuclei or their reactions hus zero net strangeness, whereas neutron stars can and almost certainly do contain hyperons.

These are the differences. Of course the properties of such systems as the hot symmetric non-strange matter produced in relativistic nuclear collisions and the cold asymmetric charge neutral and strangeness carrying matter of neutron stars are related in any comprehensive theory of matter. It is through relativistic nuclear field theory that I shall make the connection between them. This theory can be generalized to incorporate nucleons and higher mass baryon states, interacting through exchange of mesons[22]. Its coupling constants can be fixed by properties of symmetric nuclear matter. It describes numerous properties of finite nuclei $[23,24,25,25]$. It can be extended to finite temperature $[22,27]$. It can be extrapolated to hot dense matter and its composition (nucleons, deltas, and their excited states)[22], and to dense neutron star matter[28]. One and the same theory with fixed coupling constants describes:

1. Symmetric nuclear matter and the matter produced in high energy collisions when the field equations are solved subject to the constraints of isospin symmetry and strangeness conservation. 
2. Seutron star matter when the field equations are solved subject to the constraints of charge neutrality and generalized equilibrium.

Therefore we are able to conveniently characterize the neutron star matter equation of state by the compression modulus of the corresponding symmetric matter and the other properties by which the coupling constants are fixed, and shall always do so.
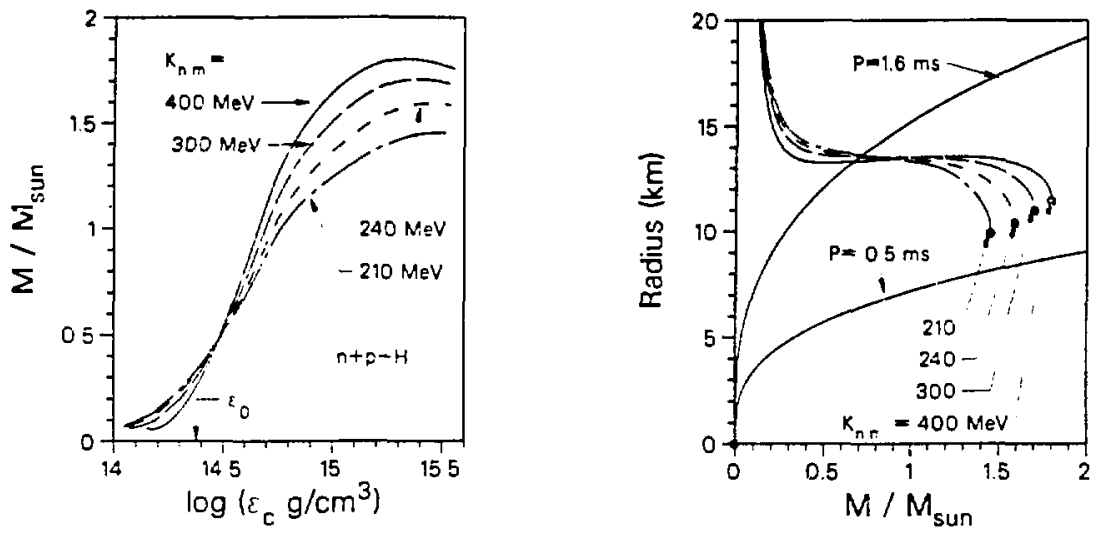

Figure 6: Generic relations for neutron stars for several equations of state as labeled according to compression $K$ (with $m_{\text {sat. }}^{*} / m=0.8$ ). For the R-M plot the limits imposed by a $1.6 \mathrm{~ms}$ and $0.5 \mathrm{~ms}$ pulsar are shown. Stars below these curves are stable for still shorter periods (see eq. (25)).

Neutron stars are not pure in neutron as their name implies, and as they were first thought of. Charge neutrality is automatically respected by pure neutron matter, but this is not the lowest energy state of dense neutral matter. Some neutrons would beta decay until equilibrium between neutrons, protons and electrons is reached. This is expressed by the relation, $\mu_{p}=\mu_{n}-\mu_{e}$ anong the chemical potentials (see eq.(28). The neutrino produced in the beta decay leaks out of the star because, being massless, it has the escape velocity, thus lowering the star's energy. Therefore we set its chemical potential to zero, because its number is not conserved in the star. At higher density as the chemical potentials increase, other thresholds are reached and additional particle species are populated. In a Fermi gas model the thresholds are found from the masses of the particles. For example, the $\Lambda$ hyperon has unit baryon charge, zero electric charge, and one negative unit of strangeness and mass $1115 \mathrm{MeV}$ 
(see table 3). As noted already, strangeness is not conserved on the time scale relevant to a star, so the chenical potential is simply $\mu_{\Lambda}=\mu_{n} \equiv \mu_{b}$, and when this exceeds the $\Lambda$ mass, this hyperon will be in equilibrium in the star and will be one of its constituents. In general the particle thresholds depend on the interactions as well. The isospin symmetry energy arising from the coupling of baryon isospin to the neutral rho meson is very important in this respect. Obviously it favors conversion of neutrons to baryons of opposite isospin projection, consistent of course with charge neutrality. For these reasons neutron star matter is very complex in composition and the Lagrangian used in nuclear field theory has to be generalized to include these complications[28]. Fig.23 slrows the result of such a general calculation for the populations of neutron star matter. For low density, the charge neutral uniform matter is almost pure in neutron, but with a small proton and electron admixture in equal numbers; when with increasing density the electron Fermi energy increases to the muon mass then the muon as well will be populated. The pion, as we discuss later, may also condense and then at densities beginning at about three times nuclear, hyperon thresholds are reached, and with further increase in density become important components of neutron star matter. Neutron stars therefore are not made of neutron matter but rather of hadronic matter that is in the lowest energy state consistent with charge neutrality. This matter we call neutron star matter. Its composition depends on the density as is easily understood from the forgoing discussion. The Lagrangian must therefore be general enough to describe these complexities and a suitable generalization of the $\sigma, \omega, \rho$ theory, used to describe nuclear matter and finite nuclei, is[28],

$$
\begin{aligned}
\mathcal{L}= & \sum_{B} \bar{\psi}_{B}\left(i \gamma_{\mu} \partial^{\mu}-m_{B}+g_{\sigma B} \sigma-g_{\omega B} \gamma_{\mu} \omega^{\mu}-\frac{1}{2} g_{\rho B} \gamma_{\mu} \tau \cdot \rho^{\mu}\right) \psi_{B} \\
& +\frac{1}{2}\left(\partial_{\mu} \sigma \partial^{\mu} \sigma-m_{\sigma}^{2} \sigma^{2}\right)-\frac{1}{4} \omega_{\mu \nu} \omega^{\mu \nu}+\frac{1}{2} m_{\omega}^{2} \omega_{\mu} \omega^{\mu} \\
& -\frac{1}{4} \rho_{\mu \nu} \cdot \rho^{\mu \nu}+\frac{1}{2} m_{\rho}^{2} \rho_{\mu} \cdot \rho^{\mu}-\frac{1}{3} b m_{n}\left(g_{\sigma} \sigma\right)^{3}-\frac{1}{4} c\left(g_{\sigma} \sigma\right)^{4} \\
& +\sum_{\lambda} \bar{\psi}_{\lambda}\left(i \gamma_{\mu} \partial^{\mu}-m_{\lambda}\right) \psi_{\lambda}^{\mu}
\end{aligned}
$$

The first line is the sum of baryon Lagrangians and the interactions with the scalar, vector and vector-isovector mesons $(\sigma, \omega, \rho)$. The spinor for the baryon species, $\mathrm{B}$, one of those listed in table 3 , is denoted by $\psi_{B}$, and $\bar{\psi}_{B} \equiv \psi_{B}^{\dagger} \gamma_{0}$ denotes the adjoint. (We use the conventions of [29].) The second line contains the Lagrangians of the scalar and vector mesons, whose interactions with the baryors give rise respectively to attraction and short range repulsion. The third line contains the Lagrangian for the isovector meson which couples to the isospin of baryons and gives rise to the charge symmetry energy. This line also contains self-interaction terms of the scalar field. The last line contains the Lagrangians for the leptons (electrons and nuons) which are important agents in the charge neutrality of neutron star matter. The sum over baryons is over the charge states of nucleons, deltas and hyperons. It is of course 
essential to keep track of the individual charge states so that charge conservation can be enforced (zero in a star and one half the baryon density in charge symmetric nuclear matter).

When the corresponding Euler-Lagrange equations are solved by replacirig the meson fields by their space-time constant mean values, and the nucleon currents by the ground state expectation generated in the presence of the mean meson fields, one obtains the so called mean field approximation (MFA). It is in this approximation that nuclear field theory has been typically solved and applied. Heaceforth the meson fields, $\sigma, \omega, \rho$, denote these mean field values. The field equations in uniform matter are,

$$
\left(i \gamma_{\mu} \partial^{\mu}-m_{B}+g_{\sigma B} \sigma-g_{\omega B} \gamma_{\mu} \omega^{\mu}-\frac{1}{2} g_{\rho B} \gamma_{\mu} \tau_{3} \rho_{3}^{\mu}\right) \psi_{B}=0
$$

whose eigenvalue of particle and antiparticle can be found as

$$
\begin{gathered}
\epsilon_{B}(k)=g_{\triangle B} \omega_{C}+g_{\rho B} \rho_{03} I_{3 B}+\sqrt{k^{2}+\left(m_{B}-g_{\sigma B} \sigma\right)^{2}} \\
\bar{\epsilon}_{B}(k)=-g_{\Delta B} \omega_{0}-g_{\rho B} \rho_{03} I_{3 B}+\sqrt{k^{2}+\left(m_{B}-g_{\sigma B} \sigma\right)^{2}}
\end{gathered}
$$

The meson field equations in uniform static matter, in which space and time derivatives can be dropped, are,

$$
\begin{aligned}
\omega_{0}= & \sum_{B} \frac{g_{\omega B}}{m_{\omega}^{2}} n_{B} \\
\rho_{03}= & \sum_{B} \frac{g_{\rho B}}{m_{\rho}^{2}} I_{3 B} n_{B} \\
m_{\sigma}^{2} \tau= & -b m_{n} g_{\sigma}\left(g_{\sigma} \sigma\right)^{2}-c g_{\sigma}\left(g_{\sigma} \sigma\right)^{3} \\
& +\sum_{B} \frac{2 J_{B}+1}{2 \pi^{2}} g_{\sigma B} \int_{0}^{k_{B}} \frac{m_{B}-g_{\sigma B} \sigma}{\sqrt{k^{2}}+\left(m_{B}-g_{\sigma B} \sigma\right)^{2}} k^{2} d k
\end{aligned}
$$

We have replaced the baryon source currents, by their groind state expectation values; the ground state is defined as having the sine' - particle momentum eigenstates with sigenvalues given by eq.(56) filled to the Fermi momentum. We will discuss shortly how the Fermi momenta of the various species, B, are to be found so that the "m is in chemical equilibrium. An example of a ground state expectaition with a familier meaning is the baryon density; $\left\langle\bar{\psi}_{B} \gamma_{0} \psi_{B}\right\rangle \equiv\left\langle\psi_{B}^{\dagger} \psi_{B}^{\prime}\right\rangle \equiv n_{B}$. For details especially 23 concerns the source for the scalar meson, see ref. [10]. In thie above equations, $I_{3 B}$ is the isospin projection of baryon charge state $\mathrm{B}$ and $k_{B}$ is the Fermi momentum of species B. Only the timc-like components of the vector fields and the iscspin 3component of the charged $\rho$ field have non-vanishing values on account of the isotropy of nuclear matter and electric charge conservation, respectively. The baryon number, charge and strangeness densitjes are

$$
\begin{aligned}
& n_{B}=\left(2 J_{B}+1\right) b_{B} k_{B}^{3} /\left(6 \pi^{2}\right) \\
& Q_{B}=\left(2 J_{B}+1\right) q_{B} k_{B}^{3} /\left(6 \pi^{2}\right) \\
& S_{B}=\left(2 J_{B}+1\right) s_{B} k_{B}^{3} /\left(6 \pi^{2}\right) .
\end{aligned}
$$


Of course the total baryon and charge densities are

$$
\begin{aligned}
& \rho=\sum_{B} n_{B} \\
& q=\sum_{B} Q_{B}
\end{aligned}
$$

Table 3: Baryon and meson states. Spin is $J$, isospin is $I$, baryon number is b, charge is $q$ and strangeness is $s$.

\begin{tabular}{c|cccccc} 
& $\begin{array}{c}\mathrm{m} \\
(\mathrm{MeV})\end{array}$ & $\mathrm{J}$ & $\mathrm{l}$ & $\mathrm{b}$ & $\mathrm{q}$ & $\mathrm{s}$ \\
\hline $\mathrm{N}$ & 939 & $1 / 2$ & $1 / 2$ & 1 & 0,1 & 0 \\
$\lambda$ & 1232 & $3 / 2$ & $3 / 2$ & 1 & $-1,0,1,2$ & 0 \\
$\mathrm{I}$ & 1115 & $1 / 2$ & 0 & 1 & 0 & -1 \\
$\Sigma$ & 1190 & $1 / 2$ & 1 & 1 & $-1,0,1$ & -1 \\
$\equiv$ & 1315 & $1 / 2$ & $1 / 2$ & 1 & 1,0 & -2 \\
$\Omega$ & 1673 & $3 / 2$ & 0 & 1 & -1 & -3 \\
\hline$\pi$ & 139 & 0 & 1 & 0 & $-1,0,1$ & 0 \\
$h^{+}$ & 494 & 0 & $1 / 2$ & 0 & 1 & 1 \\
$K^{\circ}$ & 494 & 0 & $1 / 2$ & 0 & 0 & 1 \\
$\bar{K}^{-0}$ & 494 & 0 & $1 / 2$ & 0 & 0 & -1 \\
$K^{-}$ & 494 & 0 & $1 / 2$ & 0 & -1 & -1 \\
\hline
\end{tabular}

The above equations hold at zero temperature. Finite temperature results can be obtained in the usual manner of writing the partition function for the system and carrying out the appropriate manipulations $[22,30]$. The results are simple to state. In all the expressions containing a sum over $B$, it is extended over antiparticles as well, since they are thermally excited. Their quantum numbers like baryon number, charge. jsospin, strangeness and chemical potentials are the negatives of the particles. The thermal densities for baryon charge of both baryons and antibaryons are given respectively by,

$$
\begin{aligned}
& n_{B}=\frac{2 J_{B}+1}{2 \pi^{2}} b_{B} \int_{0}^{\infty}\left(\exp \left\{\left(\epsilon_{B}(k)-\mu_{B}\right) / T\right]+1\right)^{-1} k^{2} d k \\
& \bar{n}_{B}=\frac{2 J_{B}+1}{2 \pi^{2}} \bar{b}_{B} \int_{0}^{\infty}\left(\exp \left[\left(\bar{\epsilon}_{B}(k)+\mu_{B}\right) / T\right]+1\right)^{-1} k^{2} d k
\end{aligned}
$$

and similarly for the electric charge and strangeness. Note that $n_{B}$ and $\bar{n}_{B}$ are defined as baryon charge densities, not number densities, so that if there are antibaryons present, $\bar{n}_{B}$ will be negative, counting the baryon charge rather than particle number. This is what is needed so that for example eq.(61) should count the total net baryon 
charge of the system. The thermal factor is also introduced into all the integrals over momentum in the scalar field equation above, and in the expressions below for pressure and energy density:

Compute from the ground state expectation value of the expression of the canonical stress-energy tensor,

$$
\tau^{\mu \nu}=-g^{\mu \nu} \mathcal{L}+\sum_{\phi} \frac{\partial \mathcal{L}}{\partial\left(\partial_{\mu} \phi\right)} \partial^{\nu} \phi
$$

the stress-energy for this theory and compare with eq.(1i) to read the energy density and pressure. Employ the field equations to rewrite the energy density as,

$$
\begin{aligned}
\epsilon= & \frac{1}{3} b m_{\mathrm{n}}\left(g_{\sigma} \sigma\right)^{3}+\frac{1}{4} c\left(g_{\sigma} \sigma\right)^{4}+\frac{1}{2} m_{\sigma}^{2} \sigma^{2}+\frac{1}{2} m_{\omega}^{2} \omega_{0}^{2}+\frac{1}{2} m_{\rho}^{2} \rho_{03}^{2} \\
& +\sum_{B} \frac{2 J_{B}+1}{2 \pi^{2}} \int_{0}^{k_{B}} \sqrt{k^{2}+\left(m_{B}-g_{\sigma B} \sigma\right)^{2}} k^{2} d k \\
& +\sum_{\lambda} \frac{1}{\pi^{2}} \int_{0}^{k_{\lambda}} \sqrt{k^{2}+m_{\lambda}^{2}} k^{2} d k
\end{aligned}
$$

The pressure is given by,

$$
\begin{aligned}
p= & -\frac{1}{3} b m_{n}\left(g_{\sigma} \sigma\right)^{3}-\frac{1}{4} c\left(g_{\sigma} \sigma\right)^{4}-\frac{1}{2} m_{\sigma}^{2} \sigma^{2}+\frac{1}{2} m_{\omega}^{2} \omega_{0}^{2}+\frac{1}{2} m_{\rho}^{2} \rho_{03}^{2} \\
& +\frac{1}{3} \sum_{B} \frac{2 J_{B}+1}{2 \pi^{2}} \int_{0}^{k_{B}} k^{4} d k / \sqrt{k^{2}+\left(m_{B}-g_{\sigma B} \sigma\right)^{2}} \\
& +\frac{1}{3} \sum_{\lambda} \frac{1}{\pi^{2}} \int_{0}^{k_{\lambda}} k^{4} d k / \sqrt{k^{2}+m_{\lambda}^{2}}
\end{aligned}
$$

The first step in the application of the above theory is to fix the three coupling constants, $g_{o} . g_{w}, g_{p}$. One can verify in infinite nuclear matter by inspection of the field equations that these occur only as ratios to the corresponding meson masses. So ground state properties determine only the ratios. Additionally the coefficients, $b, c$, of the scalar self-interactions must be determined. This can be done using five sat uration properties, namely the binding energy $B=\epsilon / \rho-m=16.3 \mathrm{MeV}$, baryon density, $\rho_{0}=n_{n}+n_{p}=0.153 \mathrm{fm}^{-3}$, symmetry energy coefficient

$$
\begin{aligned}
a_{s y m} & =\frac{1}{2}\left(\frac{\partial^{2}(\epsilon / \rho)}{\partial t^{2}}\right)_{t=0} \\
& =\left(\frac{g_{\rho}}{m_{\rho}}\right)^{2} \frac{k_{F}^{3}}{12 \pi^{2}}+\frac{k_{F}^{2}}{6\left(k_{F}^{2}+m^{\star 2}\right)^{3 / 2}}=32.5 \mathrm{MeV} \\
t & \equiv\left(\rho_{n}-\rho_{p}\right) / \rho
\end{aligned}
$$

The scalar effective mass of this theory, $m^{\star}=m-g_{\sigma} \sigma$, is related at saturation to the to the Landau effective mass of non-relativisitic theory $m_{L}^{\star}$, by,

$$
m_{L}^{\pi}=\left(\frac{k}{\partial \epsilon(k) / \partial k}\right)_{k_{F}}=\left(m_{s a t .}^{*}+k_{F}^{2}\right)^{\frac{1}{2}}
$$


where the Fermi momentum of the nucleons at saturation, $k_{F}$, is related to the density in the usual way, $\rho_{0}=2 k_{F}^{3} /\left(3 \pi^{2}\right)$. Landau effective mass is $\approx 0.83 m$ [31]. This which yields $m_{\text {sat. }}^{\star} / m=0.78$. Finally the compression modulus at saturation, $h$, is thought to lie in the range $200-300 \mathrm{MeV}[32,33,34,35,36]$,

$$
K=9\left[\rho^{2} \frac{\partial^{2}}{\partial \rho^{2}}\left(\frac{\epsilon}{\rho}\right)\right]_{\rho=p_{0}}
$$

Of course the hyperons are absent in the ground state because of their high masses compared to the Fermi energy of the nucleons, so they cannot be determined from the properties of ordinary nuclei.

The importance of controlling the effective mass that matter has at saturation can be understood because for given binding and saturation density it uniquely specifies the vector coupling constant through,

$$
\frac{\epsilon_{0}}{\rho_{0}}=\frac{B}{A}+m_{n}=\left(\frac{g_{\omega}}{m_{\nu}}\right)^{2} \rho_{0}+\sqrt{k_{F}^{2}+m_{\text {sat. }}^{\star 2}}
$$

This relation follows from Eqs. $(58,69)$ and the saturation condition $\partial(\epsilon / \rho) / \partial k=$ 0 craluated at $k=k_{F}$. The vector coupling in turn controls how stiff or soft at high density the equation of state is for a given fixed $K$. That is because of the quadratic term in $\omega_{0}$ that appears in energy and pressure, eqs. $(69,70)$, which from the field equation, eq.(58), gives a quadratic baryon densily behavior to the energy and pressure. Therefore for fixed $K$, the equation of state becomes stiffer at high density as $m_{\text {sat. }}^{*}$ decreases, as can be seen from the above relation. For fixed $m_{\text {sat. }}^{*}$, it becomes stiffer as $K$ increases. These are the reasons why it is important to bring both of these paraneters under control, through the freedom afforded by the scalar self-interaction terms in Eq.(54). Without this control, the application of the theory to neutron star properties or other high density phenomena can be very misleading.

Table 4: Conpling constants for several $\mathrm{K}$ and for $\mathrm{B} / \mathrm{A}=16 \mathrm{MeV}, \rho=0.15 \mathrm{fm}^{-3}$, $\mathrm{a}_{\text {sym }}=32.5 \mathrm{MeV}$ and $\mathrm{m}_{\text {sat }}^{\star} / \mathrm{m}=0.8$. [13]

\begin{tabular}{c|ccccc}
$\mathrm{K}$ & $\left(g_{\sigma} / m_{\sigma}\right)^{2}$ & $\left(g_{\omega} / m_{\omega}\right)^{2}$ & $\left(g_{\rho} / m_{\rho}\right)^{2}$ & $\mathrm{~b}$ & $\mathrm{c}$ \\
$(\mathrm{feV}$ & $(\mathrm{fm})^{2}$ & $(\mathrm{fm})^{2}$ & $(\mathrm{fm})^{2}$ & & \\
\hline 220 & 9.678 & 4.356 & 5.025 & 0.01164 & -0.001042 \\
250 & 9.216 & 4.356 & 5.025 & 0.008209 & 0.007385 \\
300 & 8.492 & 4.356 & 5.025 & 0.002084 & 0.02780 \\
350 & 7.820 & 4.356 & 5.025 & -0.001618 & 0.05015 \\
\hline
\end{tabular}

We are interested here in describing the structure of observable neutron stars, identified as pulsars. The epoch of observation is generally thousands to several millions of years after formation, and the star while still very hot by earthly standards 
$\left(10^{7}-10^{9} \mathrm{~K}\right)$, is cold on the nuclear scale of $\mathrm{MeV}\left(=1.16 \times 10^{10} \mathrm{~K}\right)$. We take $T=0$ therefore. A description of neutron star matter appropriate to this is obtained by imposing the conditions of charge neutrality and chemical equilibrium. The total charge can be computed as above. Equilibrium is obtained through the relations among the chemical potentials that correspond to the possible reactions among the constituents. Because of the long time scale of stars as compared to the weak interaction time, which is the only interaction that changes strangeniss, strangeness is not conserved in a star. In this case the strangeness chemical potential $\mu_{k}$ for positive unit strangeness is set to zero. In general, the chemical potential of particle $i$ with baryon charge $b_{i}$ and electric charge $q_{i}$ (in units of e) is given by,

$$
\mu_{i}=b_{i} \mu_{b}+q_{1} \mu_{8}, \quad \mu_{k} \equiv 0
$$

where $\mu_{b}$ and $\mu_{q}$ are the chemical potentials for unit positive baryon and electric charge and correspond to the two conserved quantities in neutron star matter. Chemical cquilibrium is then expressed as

$$
\mu_{B}=\epsilon_{B}\left(k_{B}\right)
$$

which determines the Fermi momenta, while for the leptons,

$$
\mu_{\lambda}=\left(m_{\lambda}^{2}+k_{\lambda}^{2}\right)^{1 / 2}=q_{\lambda} \mu_{Q}
$$

The solutions are the positive values of the Fermi momenta. They are to be set to zero until positive solutions exist. A description of neutron star matter is then obtajned as the self-consistent solution to a system of coupled non-linear equations in $i+N$ unknowns, as follows:

(1) three field equations for the meson fields $(\sigma, \omega, \rho)$

(2) equation for electrical neutrality $\left(\mu_{q}\right)$

(3) equation for baryon density $\left(\mu_{6}\right)$

(4) two equations for lepton Fermi momenta, $\left(k_{e}, k_{\mu}\right)$

(5) N equations for the Fermi moment a of $\mathrm{N}$ baryon species in chemical equilibrium $\left(k_{n}, k_{p}, k_{\Lambda}, \ldots, k_{\Xi}, \ldots\right)$.

When the solution is obtained the equation of state $\epsilon=\epsilon(\rho), p=p(\rho)$ can be calculated. Such a system of non-linear equations is generally cifficult to solve, which probably accounts for the fact that most applications of equations of state to neutron star structure approximate the star as either pure in neutron, or else as involving beta equilibrium in the restricted sense of equilibrium among only neutrons, protons and electrons. This is not really very useful in connection with the mass constraint on the equation of state, because both are gross approximations. Two early studies, one by Pandharipande [37], and one by Bethe and Johnson [38] did include hyperons. These 
works are non-relativistic so the equation of state explicitly violates causality at high density, and of course must be wrong even at a lower density than the point where causality is first violated. The work of Bethe and Johnson suggests a smaller fraction of hyperons than in the work of Pandharipande. However it suffers from the fact that no control was placed on the compression and the symmetry energy, even though neutron stars are highly compressed and isospin asymmetric. The hyperon fraction that $\mathbf{l}$ find is of similar importance as found by Pandharipande. The advantage of the present approach is that it is relativistically covariant, and the coupling constants of the theory are related to nuclear matter properties in a way that allows one to investigate the dependance of neutron star structure on nuclear matter properties. Is there such a dependance? Although the density at the center of a neutron star may be quite high it would be incorrect to assume that properties near saturation density are not important. This is so because the center contributes little to the mass on account of the volume element. I find that the mean density is only three to five or so times nuclear density for the star at the mass limit, and even less otherwise, depending on how stiff or soft the equation of state is. (See Fig.7). Moreover the equation of state at higher density is connected to that near saturation through continuity and causality.

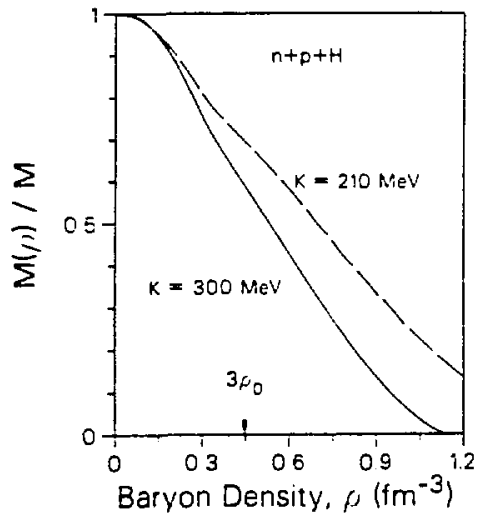

Figure 7 : Fraction of mass of that neutron star at the mass limit that is resident in matter at densities greater than $\rho$.

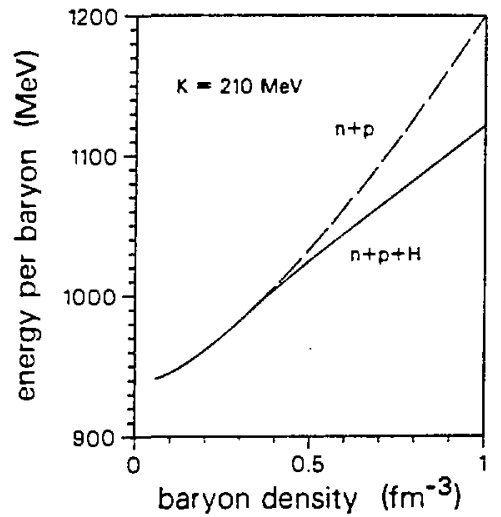

Figure 8: Compares equation of state of neutron star matter with and without hyperons included in the equilibrium composition.

We illustrate in Fig. 8 the equation of state in the form of $E / A \equiv \epsilon / \rho$ vs $\rho$ for two cases, one in which only beta equilibrium between neut rons, protons and electrons 
is taken into account, and one in which full equilibrium between all particle species to convergence is taken into account. The latter is considerably softer than the former; for the reason that the Fermi pressure of neutrons and protons near the top of the Fermi sea is relieved by allowing them to hyperonize. The corresponding results for neutron star masses is shown in Fig.9 and we see that gravity very effectively exploits the softening at higher density introduced by hyperonization. A similar reduction in limiting mass can be found between pure neutron matter and $n+p$ matter.

\section{Pion and Kaon Condensation}

Pion condensation was a subject of much investigation a few years ago, especially for symmetric nuclear matter and nuclei. Pions are more likely to condense in neutron star matter. Condensation occurs in nuclear matter if the pion energy becomes degenerate with the normal state, which might happen if the interaction is attractive and strong enough. However in neutron stars, charge neutrality favors pion condensation. This is so because as a function of increasing density, neutrons at the top of the Fermi sea will decay to proton plus electron. The electrons are fermions and their Fermi level increases with further increase in density. When the electron chemical potential (Fermi energy) becomes equal to the effective pion mass in the medium, it will be favorable thereafter for negative pions to play the role that the electrons had in preserving charge neutrality because they are bosons and can all condense in the lowest state. Thus while $\mu_{e}=\mu_{n}-\mu_{p}$ is essentially zero in symmetric matter, it is positive in neutron star matter, thus favoring the $\pi^{-}$since $\mu_{\pi^{-}}=\mu_{e}$. On the other hand the other charge states of the pion are excluded since $\mu_{x^{+}}=-\mu_{e}$ and $\mu_{x^{0}}=0$.

When pions condense the growth of the electron chemical potential with further increase in density is arrested at a value equal to the effective pion mass. This means that the electron chemical potential cannot approach the mass of the negative kaon, which is greater than that of the pion, and so they cannot condense. Condensation of the other kaon states is even less likely for the same reason as given above for the other charge states of the pion. This conclusion regarding kaon condensation is in contradiction to recent suggestion that kaons might condense at rather low density in nuclear or neutron matter[39]. However our criticism of this work, as of early work on pion condensation, is that it is based on a theory that cannot describe the normal state of nuclear matter, so how are we to rely on its prediction of an abnormal state?

Since the hyperons have charges of both signs and carry the conserved baryon charge, charge neutrality can be achieved mainly among baryons at sufficiently high density. This means that the electron chemicai will initially be an increasing function of density, will saturate if and when it becomes equal to the pion effective mass, will remain essentially saturated through a range of density, and then will decrease as the hyperon populations grow. The pions at this point will be reabsorbed. So pion condensation is an intermediate density phenomenon in neutron stars. This can be seen in Fig.23. I do not find that pion condensation has a large effect on neutron 
star structure, an example of which can be seen in Fig.10. In this calculation the effective pion mass was taken to be the vacuum mass. This probably overestimates the role of the pion. In neutron star matter, the pion experiences a repulsive s-wave interaction which would increase its effective mass. It experiences also an attractive p-wave interaction which however it must pay for by having a finite momentum. Our earlier estimate of the effective mass due to the latter is $\sim 200 \mathrm{M} \mathrm{eV} \mathrm{[40].}$

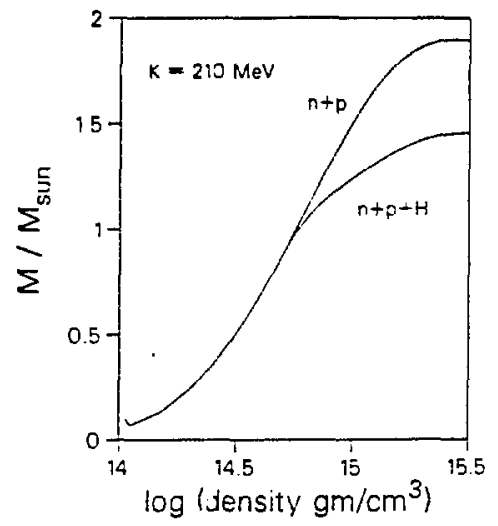

Figure 9: Neutron star masscs compared with and without inclusion of hyperons in the equilibrium composition.

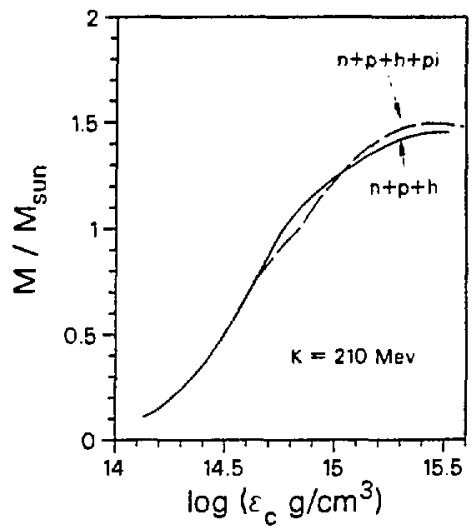

Figure 10: Neutron star masses compared with and without pion condensation.

\section{Limiting Neutron Star Mass and the Equation of state}

I have shown you that neutron stars are complex in composition. Models that treat them as pure in neutron or treat only beta equilibrium among neutrons, protons and electrons are inadequate since they overestimate the limiting mass by as much as a half a solar mass or more. This is very large in comparison with the range in which the limiting mass can lie. For a non-interacting neutron gas, which provides a lower limit because there is no repulsive force, the limiting mass is $\sim .75 M_{\odot}$. At the other extreme the hardest equations of state without full equilibrium among species give $\sim 2.75 M_{\odot}$. So the effective range in which it can fall is about 2 solar masses, and taking into account more realistic models is less than this. 


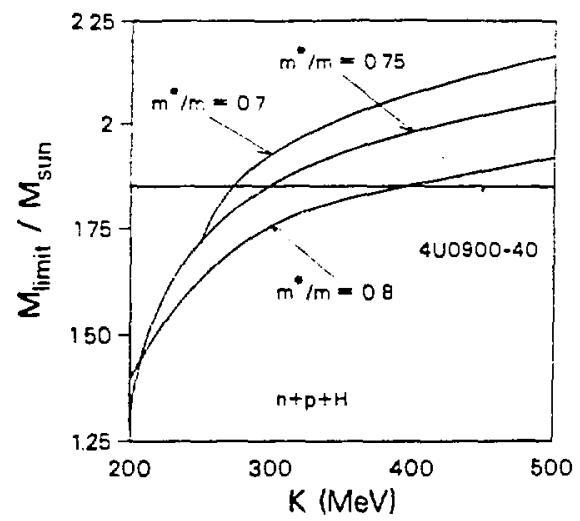

Figure 11: Limiting neutron star masses as a function of $K$ for several choices of the value of $m^{*}$ at saturation.

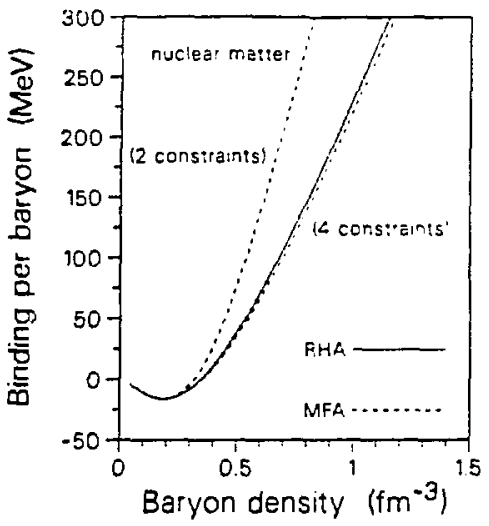

Figure 12: Equation of state with vacuum polarization (RHA, solid line) approximated in mean field theory with 2 parameter constraint at saturation, ( $B$ and $\left.\rho_{0}\right)$, and with four, $\left(K\right.$ and $m^{*}$ in addition) [41].

Do we know the limiting mass in nature? We do know of the existence of about 400 pulsars. but we know the masses of only seven[43]. This is because mass measurements are possible only for binaries. However the number of neutron stars whose masses can be measured may change dramatically in the coming years because of the discovery of fast pulsars, many of them in binaries, found within globular clusters. Globular clusters are self-bound aggregates of $10^{5}-10^{6}$ stars of which there are about 150 in our galaxy. They are especially dense in stars, and over time they expand and contract. It is believed that frequent inelastic collisions occur which form binaries. In any case, of the seven known masses, the most massive is 4 U0900-40 for which $M=1.85 \pm 0.3 M_{\ominus}$. The most accurately measured is PSR1913+16 for which $M=1.442 \pm .003$ [5]. With so few known masses it is unlikely that we know the most massive one. Therefore we can know only a lower limit on the maximum mass which translates to a lower limit on the stiffness of the equation of state. In Fig.11 I show calculated limiting masses from the above described theory as a function of compression $K$. This summarizes many such calculations as shown in Fig. 6. There is scme uncertainty owing to the fact that one of the saturation properties used to fix the coupling constants is the nucleon effective mass at saturation. While this is apparently known in a narrow range, there remains some uncertainty. To account for 


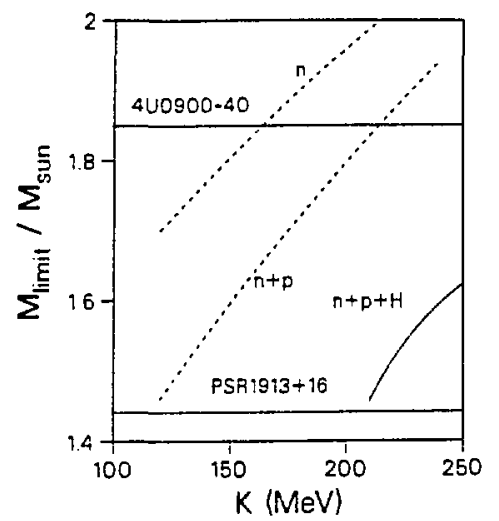

Figure 13: Limiting neutron star mass as function of $K$ for pure neutron matter and matter with neutron and proton from [42] compared to results when hyperons are taken account of (this work).

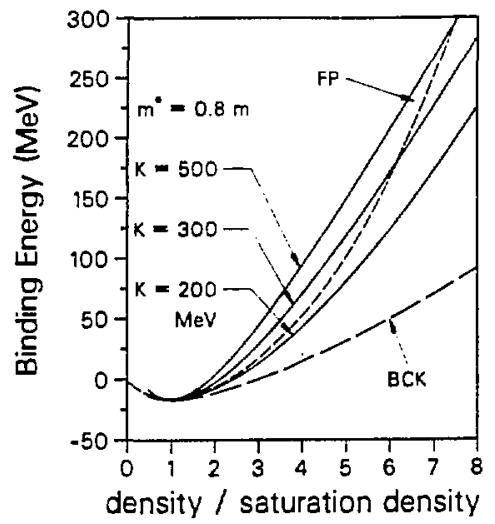

Figure 14: Comparison of various equations of state for symmetric nuclear matter. See Table 5 for further identification.

PSR1913+16 we need $K>200 \mathrm{MeV}$. To account for 4 U0900-40 at the most probable mass we need $K>300 \mathrm{MeV}$ or at the lower limit $K^{\prime}>200 \mathrm{MeV}$.

Of course it is possible to criticize the above results as being model dependent. Nonetheless the model contains a lot of physics, much more than parameterizations, or mudels based on potential interactions of nucleons which become acausal at high density, and which omit the large effect of equilibrium in the star. Recall that our theory is constrained at saturation by five nuclear properties and at all densities, but most importantly as a constraint at high densities, it is causal. These are strong constraints and should be contrasted with theories or parameterizations with fewer constraints. To emphasize this we contrast results for the linear $(b=0=c)$ and nonlinear versions of nuclear ficld theory which allow respectively three and five properties to be constrained at saturation (two and four respectively for the symmetric matter equation of state). We show in Fig.12 that in the first case the theory computed in mean field approximation can be made to agree at saturation, but no where else, with the theory which includes vacuum renormalization, which we study later. In contrast, with the additional constraints $\left(K\right.$ and $\left.m^{*}\right)$, the mean ficld theory agrees within three percent up to ten times nuclear density, although the constraints apply only at saturation density! [41]. 
Table 5: Effective stiffness for several equations of state

Description

\begin{tabular}{l} 
This work $K=500 \mathrm{MeV}, m^{*} / m=0.8$ \\
This work $K=300 \mathrm{MeV}, m^{*} / m=0.8$ \\
This work $K=200 \mathrm{MeV}, m^{*} / m=0.8$ \\
This work $K=300 \mathrm{MeV}, m^{*} / m=0.75$ \\
This work $K^{\prime}=300 \mathrm{MeV}, m^{*} / m=0.85$ \\
Friedman and Pandharipande [44] \\
BCK $[45,46] K=180 \mathrm{MeV} \gamma=2.5$ \\
\hline
\end{tabular}

Let us compare results with a recent work that purports to explain neutron star masses with much softer equations of state[42]. In Fig.13, results from that work are shown for the limiting mass of pure neutron stars and stars with only neutrons, protons and electrons. Essentially I agree with these results as far as they go. However the authors claim on the basis of their calculations that neutron stars can be accounted for with $\mathrm{K}$ as low as $120 \mathrm{MeV}$. What the figure clearly shows is that there is a substantial reduction in limiting mass in going from pure neutron to beta equilibrium. This is well known. There is a further reduction due to hyperonization as I have frequently emphasized which is shown by my calculation by the solid line. When all of this is taken account of, $K$ has to be much larger than the low value they quote. This illustration is rather typical of calculations that are incomplete with respect to the space of baryon types or which are constrained by only several saturation properties.

\section{Supernovae}

It is a fascinating thought that the heavier elements in our bodies were created in the thermonuclear burning of massive stars. Starting from a hydrogen gas and after some $10^{-}$years in the life of such a star, having reached a point in its evolution at which about a Chandrasekhar mass of iron core had been evolved, the core commences to collapse and now on a time scale of seconds, an enormous explosion occurs with a kinetic energy of $10^{51}$ ergs ( $10^{28}$ tons of TNT) expelling into the cosmos many of the elements of which we and our planet are made. Such events are so spectacular, with a brightness of $10^{10}$ times that of the sun, that some are visible to the naked eye in daylight, and several have been recorded in ancient history, such as the Crab, by Chinese observers, 900 years ago. No supernova has occurred in our own galaxy for hundreds of years, although the expected rate is about one type II every hundred years.

In the same event the inner core, some tenth or so of the total mass, forms a 
neutron star or black hole, or in the case of lighter progenitors, a white dwarf.

Why the cores of massive stars begin to collapse is easy to understand but why they explode has preoccupied scientists for several decades and a fairly detailed understanding is only now emerging.

Since it has been thought that supernovae might provide information on the equation of state of dense matter, we will spend a little time studying the problem. Of course nuclear physics is an important ingredient in understanding the entire evolution of the star, not just the final few moments. But it is this last that we shall be interested in here.

\subsection{General Features}

Supernovae involve a number of factors of comparable importance but high uncertainty. Among the factors in this connection we note:

(1). Stars in the range $8<M / M_{\odot}<100$ which evolve to be the progenitors for type II supernovae, undergo a series of nuclear fusion reactions for $10^{7}$ years, evolving from a hydrogen gas to layers of heavier elements and eventually an iron core. 'The mass and entropy of the core are crucial but highly uncertain because of the large network of nuclear reactions and their rates that must be simulated, some of them known and others highly controversial. The results of the simulation of such an evolution provide the initial conditions for the simulation of the collapse. The presupernova evolution calculations yield iron core masses in the range $\sim 1.3-$ $2.5 M_{\odot}[47]$.

(2). When thermonuclear reactions in the star have produced an iron core of approximately a Chandrasekhar mass no additional energy source is available to sustain the core against collapse under the force of gravity. Up to the critical iron core mass, it has been the pressure of the relativistic electrons that have supported the iron core, just as in white dwarfs. The collapse proceeds on the millisecond time scale.

(3). When the core reaches supernuclear density a shock originates at a radius that is interior to the iron core at a point that includes about $1 / 2 M_{\odot}$. It must propagate outward through the remaining iron core and expel most of the stars mass beyond the core, $>8 M_{\odot}$. Otherwise no supernova and neutron star; instead a black hole.

(4). The shock suffers severe energy loss as it propagates through the infalling overlaying core to the mantle that it must expel. The losses are due to the fact that the shock front dissociates nuclei loosing about $10 \mathrm{MeV}$ per nucleon in doing so.

(5). The lower limit on the iron core mass found in published stellar evolution calculations is $\sim 1.27 \mathrm{M}_{\odot}[48]$. This is marginally within the domain of possible success of the prompt explosion. Obviously from the above points, the smaller this mass the more favorable for the success of the prompt shock mechanism. Recent work has therefore focussed on attempts to make plausible a lower core mass. 
(6). Neutrino physics is as yet highly uncertain and is treated differently by the various groups doing supernova simulations. Some early claims $[46,49]$ to success were later understood to be mistaken, by failing to account for neutrino losses of the core. The excess of electron neutrinos provided an unrealistic pressure boost to the shock [50].

\subsection{Prompt Bounce and Ejection}

Five years ago, at the level of approximation employed then, it was found that supernova explosions could be simulated if the equation of state was sufficiently soft at high density $[46,49]$. This finding was then inverted and it was widely claimed that by their occurrence, supernovae inform us that the equation of state is soft. The scenario invoked at that time was that after nuclear burning had reached its end point in the pre-supernova star, having evolved about a Chandrasekhar mass of iron core, the core commenced to collapse. Upon reaching supernuclear density the inner core rebounds, sending out a shock wave that promptly expels most of the infalling material, typically 10 or more solar masses $\left(10 M_{\odot}\right)$ into a supernova explosion. In the particular case of S.1987A, about $16 M_{\odot}$ has to be ejected. With the particular approximations made in this collapse-bounce-explode scenario, sufficient explosion energy could be generated if the nuclear equation of state was assumed to be very soft. Claims for the success of the prompt mechanism have floundered in two ways.

(1) Three years ago, I showed that the equation of state that gave successful explosions was too soft to be consistent with the observed masses of several neutron stars $[51,35,52]$. The softer the equation of state the smaller the mass that can be supported against gravitational collapse, and the favored BCK equation of state cannot support the observed masses. In brief, the explosion energy was bought in those simulations at the expense of neutron star mass. We show in Fig.15 that the so-called BCK equation of state used in the Stony Brook-Brookhaven simulations, and referred to as their "preferred" one [49] is too soft to support known neutron star masses. In the one case we use a $\mathrm{Z} / \mathrm{A}$ ratio of $1 / 3$ which is on the average appropriate for the matter of the collapsing material. Later as the neutron star is formed, further neutronization occurs because it leads to a lower energy state and hence softer equation of state. Therefore we show also the neutron star masses for a $\mathrm{Z} / \mathrm{A}$ ratio more appropriate to the evolved neutron star matter, and of course the limiting mass is even less. The appropriate lepton contributions to pressure and energy are included in both cases. In neither case are the observed neutron star masses supported by this equation of state. Effectively the explosion energy in the supernova simulations has been bought at the cost of neutron star mass. It might be claimed that the BCK equation of state is intended for use $\mathrm{o}$.' at lower densities than in the core of a neutron star. However in one of the models (\# 40) reported in ref. [46] the maximum central density achieved just before bounce is $12 \rho_{0}$ so in fact it was used at very high density by its authors. It might also be claimed that it stiffens at high 


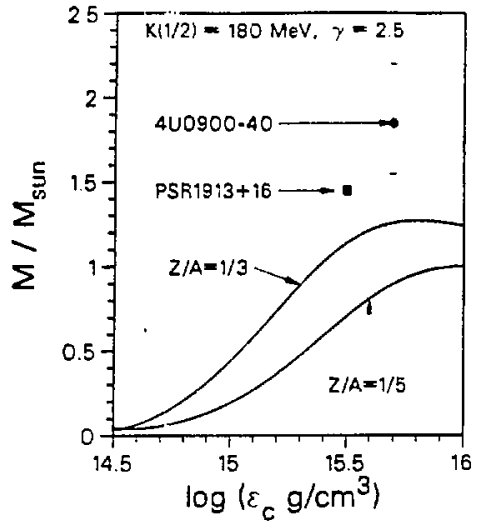

Figure 15: For the equation of state used in the supernova simulations neutron star masses are shown for two values of $\mathrm{Z} / \mathrm{A}$. The smaller corresponds more closely to that prevalent in neutron stars. In neither case can the $\mathrm{BCK}$ equation of state support stars of observed mass. [35]

density for some reason or other. However new physical effects will come into play at high density only if they are energetically favorable because physical systems arrange themselves so as to find the lowest possible energy. Of course any processes that lower the energy also soften the equation of state. Examples of this are the neutronization and hyperonization whose softening effect on the equation of state were discussed earlier. I recently read another formulation of the same idea by Bethe in discussing dense matter. "The results of these considerations are that the repulsive nuclear loops saturate, but the attractive forces increase rapidly in magnitude... These behaviors follow the general rule that repulsive interactions tend to screen themselves so as to cut down the repulsion, while attractive interactions do not." [53] Similar criticism of the claim that the equation of state effective for neutron stars structure might be stiffer than that for the supernova material and thus rescue the BCK equation of state has been made in ref.[52]. The claimed success of the prompt mechanism has also been criticized by Van Riper [54] who points out the critical importance of the core mass, which is subject to considerable uncertainty due to the complex network of nuclear reactions that build it during the pre-collapse era.

(2) It was shown by Bludman $[55,50]$ and Bruenn $[56]$ that the neutrino physics used in the papers that had claimed successful explosions had failed to account for important processes which reduce the chance for the explosion to occur promptly. The first generation of neutrinos are electron neutrinos produced by the neutronization of matter, $p+e \rightarrow n+\nu_{e}$. Because neutrino opacity goes as the square of their energy, these neutrinos would be trapped as the density approaches $10^{12} \mathrm{~g} / \mathrm{cm}^{3}$. However the early work failed to account for the down cattering of neutrinos to lower energy, for 
which the cross-sections that cause trapping are smaller. The neutrinos down-scatter because the electrons have to up-scatter on account of the Pauli blocking by occupied states. The partial deleptonization of the core during infall causes the shock to form at a deeper point in : je the iron core, meaning that the shock must propagate through a greater overlaying mass of iron. It suffers severe energy losses in doing so. This loss is easily calculated. For each $1 / 2 M_{\odot}$ of iron core through which the shock propagates the dissipation is

$$
\left(\frac{1}{2} A\right) B \approx\left(\frac{1}{2} 10^{57}\right) \times 10 \mathrm{MeV}=5 \times 10^{57} \mathrm{MeV}=8 \times 10^{51} \text { ergs }
$$

where $B$ is the nuclear binding energy and $A$ is the number of nucleons in a solar mass. This dissipation energy is about five times the entire kinetic energy of the expiosion. (That of SN1987A is estimated to be $1-310^{51}$ ergs.) Bludman finds that even uing the very soft equation of state of $\mathrm{BCK}$ and a small iron core, which is favorable to the prompt ejection, the explosion fails with these improvements in neutrino physics.

There is now universal agreement among those who have studied the problem. including now the authors of the original papers [5i] that the prompt mechanism fails when the best physics to date is incorporated. Perhaps it fails just because it is prompt! It is vulnerable to energy losses on the one hand and on the other its time scale is too short for this energy loss to be replenished from the tremendous energy that will be released ultimately as the proto-neutron star sinks into its gravitational potential. (On the time scale of the prompt scenario, the proto-neutron star is still very hot and has a radius of $\sim 100 \mathrm{~km}$, whereas it will finally shrink to $\sim 10 \mathrm{~km}$, with consequent further release of energy due to gravitational binding.)

Typically the shock is overwhelmed by the energy loss caused by the heating and disintegration of nullei as it propagates. As noted above, for each $1 / 2 M_{\odot}$ of iron that the shock has to propagate through: it looses about five times as mich energy as is typically seen in the kinetic energy of the supernova explosion. Yet a hundred times this energy will soon be released in binding energy of the neutron star. It will appear mostly in neutrinos because they can escape on a shorter time scale than photons. Evidently since stars do explode, nature finds a way of converting enough of this neutrino energy into explosion energy. This brings us to the next scenario.

\subsection{Neutrino Reheating Explosion}

J. Wilson discovered this mechanism[58], and others [59] have contributed very importantly to recent refinements. As we just saw, the shock typically stalls and turns into an accretion shock at several hundred kilometers. Most of the material of the pre-supernova star is still falling inward toward this point. If it is not expelled, the star will collapse to a black hole. For the next several hundred milliseconds, after core bounce, the matter behind the stalled shock is heated by partial absorption of an intense neutrino flux from the evolving neutron star as it gives up binding energy. 
The heated material expands pushing the accretion shock front out to greater distance and leaving a hot rarefied bubble region in its place. A weak explosion may be the result of this. However, more likely this matter again stalls, but now at greater distance where the neutrino flux is smaller so that neutrino absorption is reduced. Meanu hile because of expansion the matter has cooled. Together with these factors and under the force of the infalling matter, the shock front would be pushed in again and the cycle would repeat itself since at the new closer position reheating by neutrino absorption would reoccur. But the important new realization is that for the next few hundred milliseconds there is another energizing mechanism, neutrino-antineutrino annihilation in the bubble region which raises the pressure in this region. It is only a matter of time until there is sufficient energy deposition to unbind the material at the stalled shock and with surplus energy with the resulting ejection in a supernova. It is now believed by a number of workers in the field that this is a fairly accurate description of how stars explode. A strong case for it, and the need for the hot bubble is discussed by Colgate[60]. It is a mechanism which unique among earlier ones, couples some of the binding energy being released by the proto neutron star to the outer lavers over an extended time.

In this rather long term mechanism. matter spends much time at sub- nuclear density: Nuclear density plays a role in the explosion only at the time of high compression just hafore the bounce. It will not be surprising therefore if its effects are masked by the long evolution after the (stalled) bounce.

As it appears at present, the prompt mechanism may ultimately be found to produce supernovae in the lightest progenitors and the late-tims neutrino reheating mechanism to be responsitle for the explosion of all the others. It is hoped and expected that this long outstanding problem will be solved within the next year or two.

\section{Conclusions for supernovae:}

1. It has not been established that supernovae provide a constraint on the equation of state of nuclear matter as was frequently quoted. There are many other factors involved of comparable importance but high uncertainty.

2. It is not presently understood why stars explode, though the late time mechanism is promising.

\section{Vacuum Polarization and Neutron stars}

So far the only known effective relativistic field theory that can describe nuclear matter and finite nuclear properties is the scalar-vector-isovector $(\sigma, \omega, \rho)$ theory that we have described above. That it provides a good description of numerous properties of nuclear matter and finite nuclei lends support to its use in deriving the properties of matter at high energy density, but below the expected transition to a quark-gluon plasma. It is known how to incorporate vacuum renormalization $[61,27]$, and this 
has been done in several recent works on nuclear matter and neutron stars[62, 63]. It so far was not been studied systematically in a way that preserves the five important properties of nuclear matter at saturation, the binding, density, compression modulus, effective mass and symmetry energy until our recent work[41]. Therefore it had not been possible to disentangle the renormalization effects from those produced by shifting nuclear matter properties. Moreover, vacuum polarization in neutron star matter that is in generalized beta equilibrium had not been investigated previously, except in the chiral-sigma mode][64], which seems incapable of describing the normal ground state of finite nuclei, producing instead a bubble configuration [6.5]. Here we discuss such a systematic investigation of nuclear and neutron star matter for the $\sigma, \omega, \rho$ theory. This requires the form of the theory in which cubic and quartic selfinteractions of the scalar field are included[66], and renormalized, for they, together with the nucleon interaction with the scalar, vector and vector-isovector mesons, permit the five saturation properties to be controlled $[22,67,10,68]$.

Most calculations based on nuclear field theory have been done in the mean field approximation. However, as is well known, the presence of matter alters the vacuum, by altering the masses of antiparticles. The energy of the filled sea therefore shifts with density. There are well known procedures for renormalizing the theory with respect to nucleon, and scalar and vector mesons[27]. So far it is not known how to renormalize the vector-isovector meson, and we shall regard as phenomenological the energy contributed to asymmetric matter by the coupling of this meson to the isospin current. with this coupling chosen to reproduce the empirical symmetry energy coefficient.

With the inclusion of vacuum renormalization energies, the energy density is given by

$$
\epsilon_{R H A}=\epsilon_{M F A}+V_{N}+V_{\sigma}
$$

where $\epsilon_{M F A}$ is the energy density calculated in MFA, eq. (69). The last two terms represent the contributions from renormalization of the nucleon and scalar meson[27], and are given by,

$$
\begin{aligned}
V_{\sigma}= & \frac{m_{\sigma}^{4}}{(8 \pi)^{2}}\left[\left(1+\phi_{1}+\phi_{2}\right)^{2} \ln \left(1+\phi_{1}+\phi_{2}\right)-\left(\phi_{1}+\phi_{2}\right)-\frac{3}{2}\left(\phi_{1}+\phi_{2}\right)^{2}\right. \\
& \left.-\frac{1}{3} \phi_{1}^{2}\left(\phi_{1}+3 \phi_{2}\right)+\frac{1}{12} \phi_{1}^{4}\right] \\
V_{N}= & -\frac{m_{n}^{4}}{4 \pi^{2}}\left[(1-x)^{4} \ln (1-\chi)+\chi-\frac{7}{2} \chi^{2}+\frac{13}{3} \chi^{3}-\frac{25}{12} \chi^{4}\right]
\end{aligned}
$$

where

$$
\phi_{1}=\frac{2 b m_{n} g_{\sigma}^{3} \sigma}{m_{\sigma}^{2}}, \quad \phi_{2}=\frac{3 c g_{\sigma}^{4} \sigma^{2}}{m_{\sigma}^{2}}, \quad \chi=\frac{g_{\sigma} \sigma}{m_{n}}
$$

and $m_{n}$ and $m_{\sigma}$ are the nucleon and $\sigma$ mass. The approximation which includes the vacuum renormalization is known as the relativistic Hartree approximation (RHA)[27]. 
The field equations can be found either as the solutions of the Euler-Lagrange equations, or equivalently, as the values that minimize the energy density at fixed baryon density. They are the same as those written earlier, eqs. $(58,59)$ except that the equation for the scalar field, eq. (60), is modifies by the polarization terms above,

$$
\begin{aligned}
m_{\sigma}^{2} \sigma=-b m_{n} g_{\sigma}\left(g_{\sigma} \sigma\right)^{2}-c g_{\sigma}\left(g_{\sigma} \sigma\right)^{3}-\frac{\partial}{\partial \sigma}\left[V_{N}+V_{\sigma}\right] \\
+\sum_{B} \frac{2 J_{B}+1}{2 \pi^{2}} g_{\sigma B} \int_{0}^{k_{B}} \frac{m_{B}-g_{\sigma B} \sigma}{\sqrt{k^{2}+\left(m_{B}-g_{\sigma B} \sigma\right)^{2}}} p^{2} d p
\end{aligned}
$$

The pressure is given in RHA by,

$$
p_{R H A}=p_{M F A}-V_{N}-V_{\sigma}
$$

where $p_{M F, A}$ is the pressure previously obtained, eq. (70).

As before, the five important properties of nuclear matter, mentioned earlier can be used to fix the coupling constants $g_{\sigma} / m_{\sigma}, g_{\nu} / m_{\omega}, g_{\rho} / m_{\rho}$, and the parameters of the scalar self-interactions, $b$ and $c$. In uniform matter, it is only the ratio of coupling constant to mass on which the theory depends, except for the scalar mass, which appears independently in the vacuum renormalization energy. For that mass we take $m_{0}=600 \mathrm{MeV}$. The binding, saturation density and symmetry energy coefficient are relatively well known[69]. The compression modulus has been the subject of considerable debate in the last several years. However a recent analysis of a broad body of evidence[35], and recent new experiments on the giant monopole resonance[36] both suggest that $K \approx 300 \mathrm{MeV}$, and this is the value we adopt throughout.

Table 6: Nucleon-meson coupling constants, corresponding to $K=300 \mathrm{MeV}, B / A=$ $16.3 \mathrm{MeV}, \rho_{0}=0.153 \mathrm{fm}^{-3}, a_{\text {sym. }}=32.5 \mathrm{MeV}, m_{\text {sat. }}^{\star} / m=0.78$.

\begin{tabular}{c|ccccc} 
& $\begin{array}{c}\left(g_{\sigma} / m_{\sigma}\right)^{2} \\
\mathrm{fm}^{2}\end{array}$ & $\begin{array}{c}\left(g_{\omega} / m_{\omega}\right)^{2} \\
\mathrm{fm}^{2}\end{array}$ & $\begin{array}{c}\left(g_{\rho} / m_{\rho}\right)^{2} \\
\mathrm{fm}^{2}\end{array}$ & $\mathrm{~b}$ & $\mathrm{c}$ \\
\hline \hline MFA & 9.031 & 4.733 & 4.825 & .003305 & .01529 \\
RIA & 9.249 & 4.732 & 4.823 & .005723 & .000601 \\
\hline \hline
\end{tabular}

We first assess the effect of the vacuum polarization on the binding energy of normal nuclear matter, by adjusting the coupling constants so that the saturation properties discussed earlier are reproduced in both the mean field (MFA) and the relativistic Hartree approximation (RHA). The corresponding coupling constants are given in Table 4, and the comparison of the two approximations can be seen in Fig. 16, for both nuclear matter and pure neutron matter. The equation of state in both approximations are surprisingly alike, differing at most by about three percent even at ten times nuclear density. This is a very encouraging result, since in the many 
applications of the theory to finite nuclei and neutron stars, the MFA has been employed up till now. Next we show in Fig. 17 the separate contributions to the equation of state arising from the two-body, as well as the three and four-body terms in the energy and the two contributions $V_{N}$ and $V_{\sigma}$ of the vacuum renormalization. Aside from the region near saturation, the three and four-body terms, and the vacuum renormalization energies are all rather independent of density. They become relatively unimportant compared to the two-body energy at higher density. The scalar renormalization energy, $V_{\sigma}$, is particularly small. It can be regarded as an advantage of the $\sigma, \omega, \rho$ model that the vacuum polarization energies are relatively constant, and have so little effect when the coupling constants are renormalized so that the five saturation properties are reproduced. The implication of the above result is that the neglect of vacuum renormalization, which in principle could produce drastic changes in the nuclear properties, is unlikely to be very important in many applications to finite nuclei and to neutron star structure.

The solution to the above system of equations, the field equations, constraint equations and conditions of chemical equilibrium among the baryon species and leptons can be presented as the value of the three field variables and two chemical potentials. (We represent the scalar field by the effective nucleon mass $m^{*}=m-g_{\sigma} \sigma$.) We show such solutions for the fuli case of general equilibrium in neutron star matter, which contains nucleons, hyperons and leptons in Fig. 18, and in the case that hyperons are absent, and beta equilibrium exists between neutrons protons and leptons, in Fig. 19. The Fermi momenta of the baryons and leptons can be reconstructed from these quantities through eqs. (75-77). There are two points of special interest. In the case that hyperons are absent, the field strength of the (time-like component) of the neutral rho-meson, $\rho_{03}$, is a monotonic increasing function of density. Recall that the isospin symmetry energy density arising from this meson is

$$
\epsilon_{\rho}=\frac{1}{2} m_{\rho}^{2} \rho_{03}^{2}
$$

where the $\rho$ field strength, $\rho_{03}$ is given in terms of the isospin projection of the various constituents and their densities by Eq.(59). In contrast to the above behavior of the $\rho$ meson field strength in neutron-proton matter, or pure neutron matter, the hyperons cause it to saturate, as revealed in Fig. 18. This happens for two reasons: As hyperons become prevalent in the star, they enter in such isospin states that tend to reduce the large negative isospin of the dominant population, the neutrons, in accord with the above symmetry energy, always consistent with the absolute constraint of charge neutrality imposed by the long-range Coulomb force. Second, as we discuss later, the hyperons are more weakly coupled to the meson fields than are the nucleons. For both reasons, hyperons tend to cause the contribution to the symmetry energy of the rho meson to saturate.

A second feature of interest in Figs. 18 and 19 is the saturation of the electron chemical potential, $\mu_{e}$ by the hyperons. This occurs because charge neutrality can be 
achicved more economically among hyperons and nucleons when the electron chemical potential beconcs of the order of the their mass difference, than among nucleons and additional relativistic leptons. The saturation of $\mu_{e}$ by hyperons in neutron star matter has special significance for the possible condensation of negative pions. When $\mu_{e}$ exceeds the effective nass of the pion in matter ${ }^{2}$, then the negative pion is energetically more favorable for maintaining charge neutrality than additional relativistic electrons. This is because pions are bosons, and they can all condense in the lowest energy state. In this event, pions saturate $\mu_{e}$. However, from Fig. 18, the hyperons salurate the electron chemical potential at $\mu_{\mathrm{e}}<190 \mathrm{MeV}$. Therefore pions cannot condense if their effective nuass in matter exceeds this value. On the other hand, pions experience a repulsive s-wave and attractive p-wave interaction with nucleons. The attraction in the latter case has to be bought at the expense of finite momentum. In neutron-proton beta stable neutral matter, pions cause $\mu_{e}$ to saturate at $177 \mathrm{MeV}$ [40]. The smallest plausible value of the pion effective mass is its vacuum value. So pions may condense in ncutron star natter over a certain finite interval of density for which $\mu_{\mathrm{e}}$ would otherwise exceed the pion effective mass. The interval is of finite extension because as we see in Fig. 18, $\mu_{\mathrm{e}}$ reaches a maximum and then decreases as byperon populations increase. Assuming, as a maximum estimate of the effects of pion condensation, that pions condense at their vacuum mass, the solution of the equations for neutron star matter are shown in Fig. 20. The flateau region in $\mu_{e}$ is caused by the pion condensation which arrests the growth of $\mu_{e}$. Of course the use of a single valued pion effective mass is an approximation, and the use of the vacuum mass provides an estimate of the maximum effect of pions in neutron star matter, according to the above discussion.

The equation of state of stable, charge-neutral, neutron star matter is shown in Fig. 21, with and withou1 vacuum renormalization. We also show by way of contrast, the equation of state for pure neutron matter, with vacuum renormalization. The equation of state for stable ncutron star matter lies considerably below that for pure neutron matter. This softening is a result of the conversion of energetic nucleons to hyperons and the relaxation into an equilibrium population of many baryon species, in contrast to the non-equilibriun population of only the neutron in pure neutron matter. A study of these results reveals how inappropriate is the idealization of pure neutron matter for neutron stars. This is reinforced by an examination of the composition of neutron star matter, including the renormalization of the vacuum, which is shown in Fig. 22. The threshold for the first hyperon lies little above two times nuclear density. In principle other baryon resonances like the delta could also be present, but under the assumption of equal coupling of nucleons and deltas, they do not appear in the density domain of neutron stars. For very low densities, neutron star matter is almost pure in neutron. However the electron and proton populations rise rapidly, even below nuclear density. These populations are initially equal, as a

\footnotetext{
${ }^{2}$ We shall refer to the dispersion relation energy, $k_{0}=\sqrt{m_{8}^{2}+k^{2}+\operatorname{II}\left(k_{0}, \mathbf{k}\right)}$ evaluated at $k_{0}=\mu_{e}$, as the effective pion mass
} 
result of the constraint of charge-neutrality. (Excess charge would be blown off a star by the Coulomb force, which is so much stronger than the gravitational.) At densities still below nuclear density, the increasing chemical potential of the electrons makes it favorable for muons to replace electrons at the top of the Fermi distribution. At a little more than twice nuclear density, the increasing nucleon Fermi energy makes it favorable for the $\Sigma^{-}$to replace a neutron and lepton at the top of their respective Fermi distributions. Thereafter other thresholds are reached, the $\Lambda, \Sigma^{0}, \Xi^{-}$, and so on. Under the assumption of equal coupling of nucleon and delta to the meson fields, the latter does not appear in the density domain of neutron stars. This is because the most favored charge state is the $\Delta^{-}$, because it can replace a high momentum neutron and electron, but it has isospin projection $-3 / 2$, the same sign as that of the dominant species, the neutron, but three times the magnit ide. It is therefore highly isospin unfavored. The lepton populations decrease at densities above the hyperon thresholds. as these populations increase. Eventually charge neutrality is achieved, mainly among the baryons themselves. As we have mentioned before[10], this could effect the electrical conductivity of a neutron star, and hence the lifetime of its magnetic field and active life as a pulsar.

In the case that the effective pion mass is assumed to equal its vacuum mass, they condense in neutron star matter. The populations in this case are shown in Fig. 23. The principle difference caused by pion condensation is that because they are bosons, they quench the lepton populations, and by altering the manner in which charge neutrality can be achieved, rearrange the hyperon populations. At sufficiently high density, the pions themselves are quenched by the hyperons. At moderate density above nuclear density, the pions are almost as populous as protons, being the principle agent of charge neutralization in that domain.

Such large hyperon populations in dense neutron star matter are supported by the non-relativistic calculations of Pandharipande [37] but not by Bethe and Johnson [38]. However since these early works, it has been realized that when sich calculations based on two-body interactions are carried to convergence, nuclear matter saturates at twice the empirical density [70]. Moreover, even though neutron stars have dense interiors, and are the most isospin asymmetric objects known, the compression modulus and symmetry energy were not listed among the seven constraints on the early work [38]. As we show later, about half the mass of the heaviest neutron stars is composed of matter in the lower density domain below $3 \rho_{0}$, so that such uncertainties as those mentioned are quite important for neutron star structure. Moreover, such uncertainties propagate, by continuity, into the high density domain. The ordering of thresholds for the higher baryon states in both of the above works [37, 38] suggests that the symmetry energy at higher density becomes small in comparison with that expected from the coupling of baryons to the rho-meson, as was discussed elsewhere $[10]$.

The equation of state of neutron star matter in general equilibrium, represented as pressure as a function of energy density, is compared in Fig 24 with that 
of beta stable matter involving only neutrons, protons and leptons, and with pure neutron matter. Also the case where pions condense is shown. In all cases the vacuum renormalization is included. We note that the causal limit, $p=\epsilon$, is respected by these relativistic theories, in contrast to theories of matter described in the Schroedinger approach.

The equations of star structure need to be integrated to $p=0$. Therefore we supplement the high density equation of state of this work by the appropriate equation of state of the lower density domains of matter $[12,11]$, as in our previous work $[10,68]$.

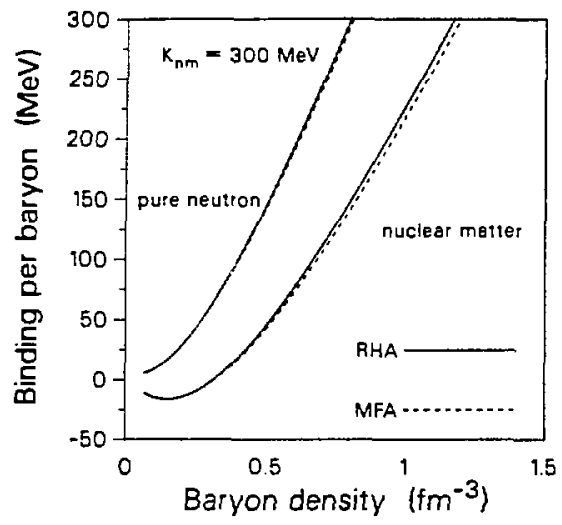

Figure 16: Binding energy, $B / A=$ $\epsilon / \rho-m$, of nuclear and pure neutron matter as a function of density, computed with and without vacuum renormalization, denoted as RHA and MFA respectively. The corresponding nucleär matter properties are listed in Table 6. [41]

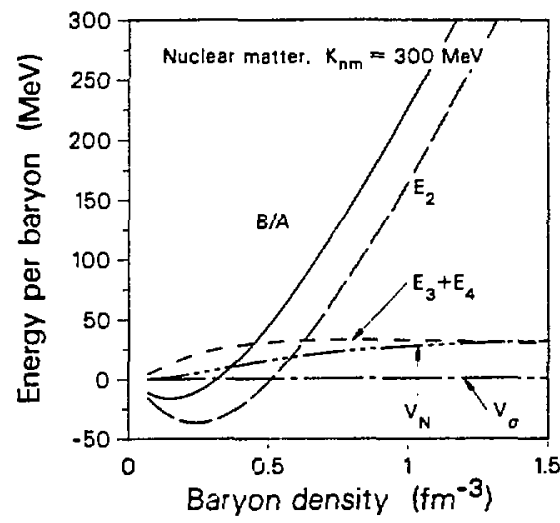

Figure 17: For nuclear matter, the separate contributions of the two-, threeand four-body terms and the vacuum polarization energies. [41]

\section{Other Dependences of Neutron Stars}

We have calculated the families of neutron stars both with and without vacuum polarization. Again the coupling constants are those of table 6 , which give identical nuclear matter properties in both approximations. The effect on neut ron star masses is found 


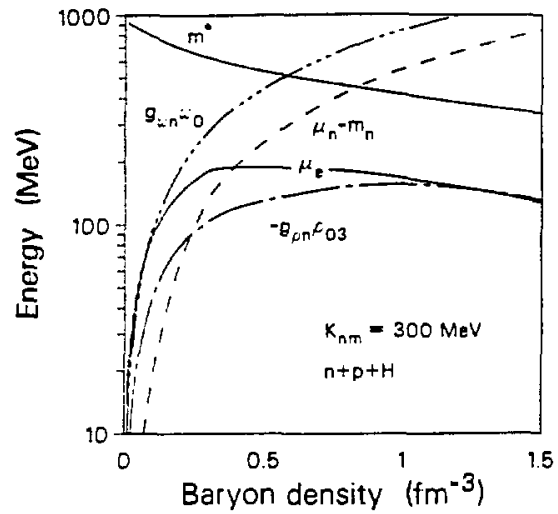

Figure 18: Solution for neutron star matter for the general case of equilibrium among $\mathrm{n}, \mathrm{p}$, hyperons and leptons. The quantities plotted are the $m^{\star}=m-g_{\sigma} \sigma$, vector and rho fields, $\omega_{0}, \rho_{03}$ and the two chemical potertials for charge, and baryon number, $\mu_{e}, \mu_{n}$, respectively. [41]

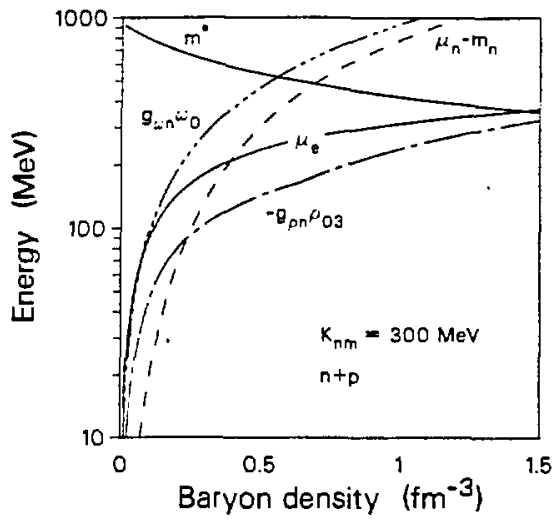

Figure 19: Similar to Fig. 18, for beta equilibrium among $n, p$ and leptons. [41]

to be negligible, not because the vacuum polarization energies are negligible, but because the coupling constants in both cases give the same five saturation properties of nuclear matter and therefore yield essentially the same equation of state over the entire relevant density range as was shown in fig. 16. Consequently all of our earlier investigations of neutron star structure, and in particular the limits that we found to be imposed on the equation of state by neutron star masses, stand unchanged. In the remainder of the paper we shall show the results only for the RHA (vacuum polarization included).

The properties of symmetric matter at saturation do not, of course, yield any information about the hyperon-meson couplings. We investigate first the uncertainty associated with this. Moszkowski[71], using quark counting arguments, suggests that these couplings should be reduced over that of nucleons by

$$
g_{H} / g_{N}=x=\sqrt{2 / 3}
$$

On the other hand from evidence on hypernuclei, Walker[72], suggests that $x$ should be smaller, around 0.4 . No matter what the coupling strength, even if free, hyperons 


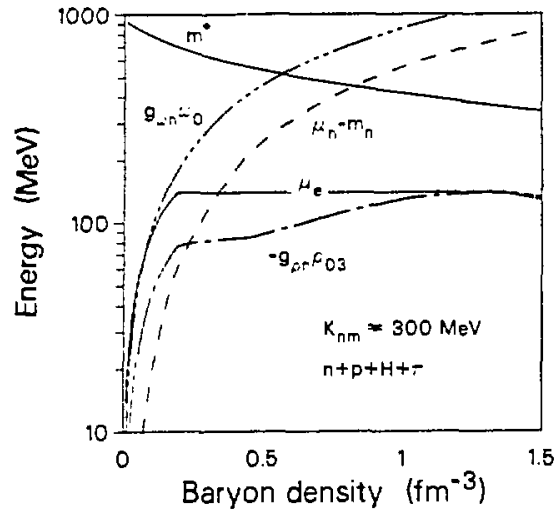

Figure 20: Similar to Fig. 18, for general equilibrium among $n, p$, hyperons, pions and leptons. [41]

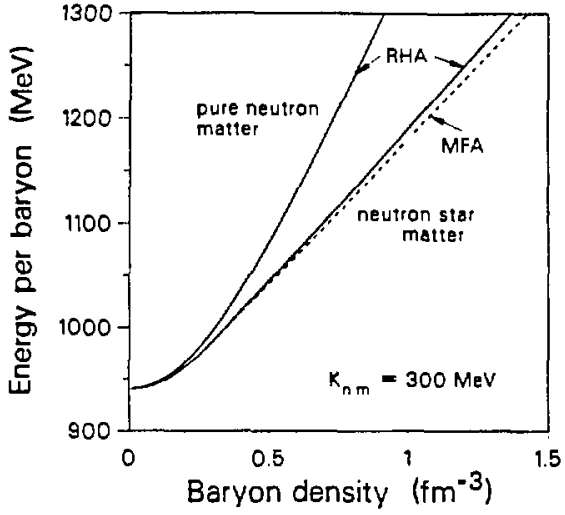

Figure 21: Energy per nucleon for neutron star matter with and without vacuum polarization (RHA and MFA). Also shown is pure neutron matter in RHA. Properties of the corresponding nuclear matter and the coupling constants in Table 6. [41]

are expected to appear in neutron star matter[73]. We compare three cases in Fig. 25. A modest reduction in limiting mass results from the reduction of $x$ from universal coupling $(x=1)$ to the value suggested by Moszkowski. The value suggested by Walker would lead to an even greater participation of hyperons in dense matter, with the first threshold occurring at little over twice nuclear density. This is because the vector repulsion is more important than the scalar attraction, and the weakening of the coupling constants is therefore favorable to hyperons in the dense region. We suspect that this last coupling is probably too drastic an estimate of the relative hyperon to nucleon couplings to mesons, and shall use Moszkowski's coupling in the remainder of the paper. This is also the coupling employed for the calculation of the equations of state shown in Figs. 21 and 24.

We summarize the effects on the limiting neutron star mass of uncertainties in the hyperon couplings over a range of $K$ and a fixed $m_{\text {sat }}^{*}$ in Fig. 26. From Fig. 11 one could infer approximately the limiting mass at another $m^{\star}$. The effect of hyperons is always to reduce the limiting neutron star mass. The amount of the reduction can vary compared to a pure neutron star approximation by up to a solar mass, but not less than $1 / 4$ solar mass 


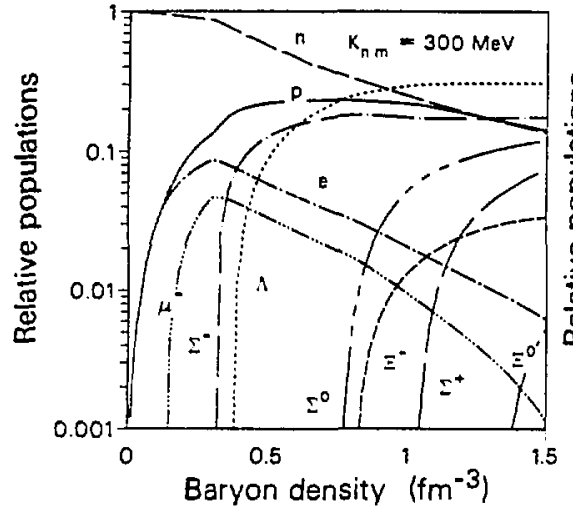

Figure 22: Composition of stable, charge-neutral (neutron star) matter, represented as a fraction of baryon density for the various components. This calculation includes vacuum polarization (RHA), whose equation of state is shown in Fig. 21. [41]

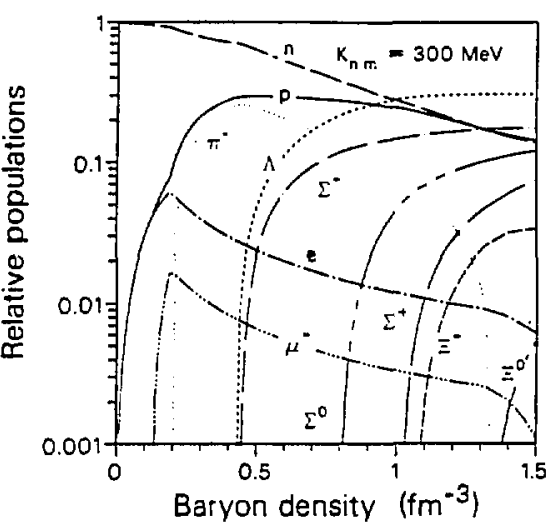

Figure 23: Relative populations in neutron star matter as a function of density [41]. Pions are included.

Next for the three equations of state shown in Fig. 24, we show the corresponding star masses in Fig. 27. Protons make their appearance in neutron star matter below the central density of the lightest neutron stars, so the pure neutron stars and the beta stable stars are shifted in mass with respect to each other, with the latter being lighter because of the softer equation of state. The first hyperon threshold occurs a little above $2 \rho_{0}$ and is plainly visible both in the equation of state (Fig. 24) and in the star mass as a function of central density. At the limiting mass, the effect of beta equilibrium is a reduction of mass of about $\frac{1}{3} M_{\odot}$ and hyperons cause a further reduction of about the same amount. Here we use the hyperon coupling suggested by Moszkowski. As shown above, the effect would be even larger were the coupling suggested by Walker used. In either case, the effects are larger the smaller the compression modulus of symmetric matter[74].

It is sometimes claimed that neutron star masses are not sensitive to the density domain of normal nuclei, and are therefore insensitive to nuclear matter properties. This is manifestly untrue for the lighter stars, since their central densities are not high. It is also not true for stars at the limiting mass. Although the cores of neutron stars at the limiting mass are dense, the mass of a star is not dominated 


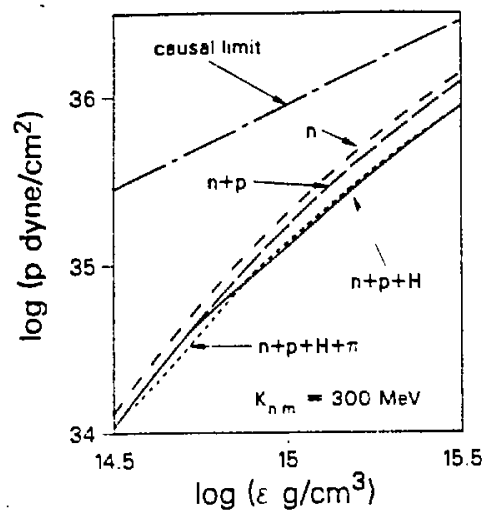

Figure 24: Equation of state for neutron star matter in general equilibrium, $(n+p+H)$, beta stable matter in which hyperons are ignored, $(n+p)$, and pure neutron matter, (n). Calculations include vacuum polarization. Nuclear density is $\log \left(\epsilon_{0} \mathrm{gm} / \mathrm{cm}^{3}\right) \approx$ 14.4. [41]

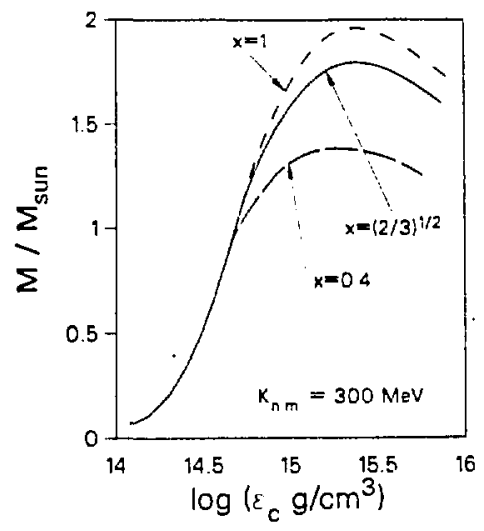

Figure 25: Neutron star mass as a function of central density for three choices of hyperon coupling (see Eq.86 ). Computed in RHA. [41]

by the central density. The reasons are two: the star is three dimensional and relativistic. The consequence is illustrated in Fig. 7 . There we show the fraction of mass $M(\rho) / M$ that is composed of matter at densities greater than $\rho$. What we find for $K=300 \mathrm{MeV}$ is that about $50 \%$ of the mass is composed of matter at densities less than $3 \rho_{0}$ while the central density is $\rho_{c}=7.2 \rho_{0}$, so that the limiting mass star is dominated neither by low nor high density. Besides, we have explicitly shown elsewhere how the limiting star mass depends on such saturation properties as $K$ and $m_{\text {sat. }}^{*}[34,35]$. This is so, not only because an appreciable portion of the stars mass is contributed by matter near saturation density, as shown in the figure, but also because the equation of state is everywhere specified by its coupling constants, which determine alike the saturation properties as well as the high density behavior of the equation of state. One may argue with the model of nuclear matter but not with the inextricable connection of all domains of the equation of state through the coupling constants of theory. A disadvantage of equations of state based purely on a parameterization is that such a connection is absent. 


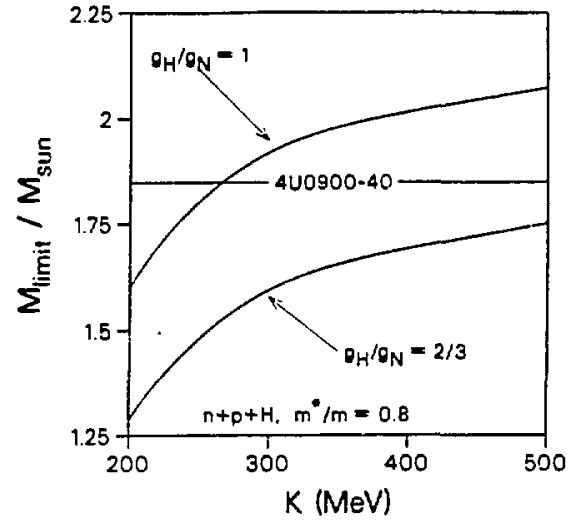

Figure 26: As a function of $K$ the limiting mass at two values of the hyperon to nucleon coupling. [41]

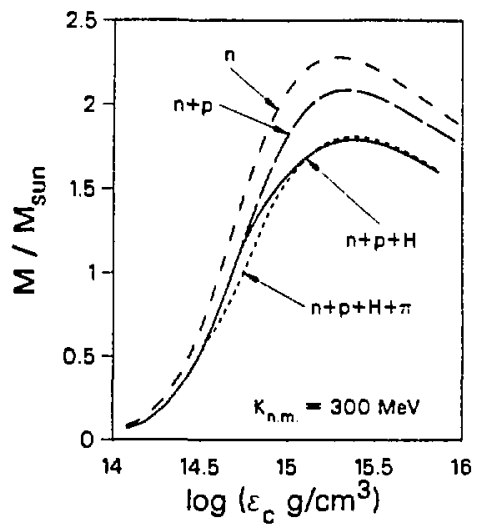

Figure 27: Neutron star mass computed for the four equations of state shown in Fig. 24. This shows how pure neutron matter overestimates the star mass. [41]

\section{Redshift, Binding etc.}

It is possible as more observational data is gathered, that the gravitational redshift and the mass of a gamma ray burster (neutron star) will become available for the same star. If the spectral line between 300 and $500 \mathrm{KeV}[75,76\}$ can be unambiguously associated with the gravitational redshift of electron-positron annihilation at the star's surface, an interesting additional constraint on the equation of state will be imposed. We show the gravitational redshift as a function of neutron star mass in Figs. 28. The surface redshift is defined as the fractional shift in the wave-length of light that is emitted from the star surface,

$$
z=\frac{\Delta \lambda}{\lambda}=e^{\lambda(R)}-1
$$

where the radial metric function, $\lambda(r)$, is given by,

$$
e^{-2 \lambda(r)}=1-\frac{8 \pi}{r} \int_{0}^{r} c(r) r^{2} d r=1-\frac{2 M}{r}, \text { for } r>R
$$

where $M$ is the star mass, $R$ its radius and $c(r)$ is the radial distribution of energy density in the star, all of which are obtained as solutions to the Oppenheimer-Volkoff equations. 


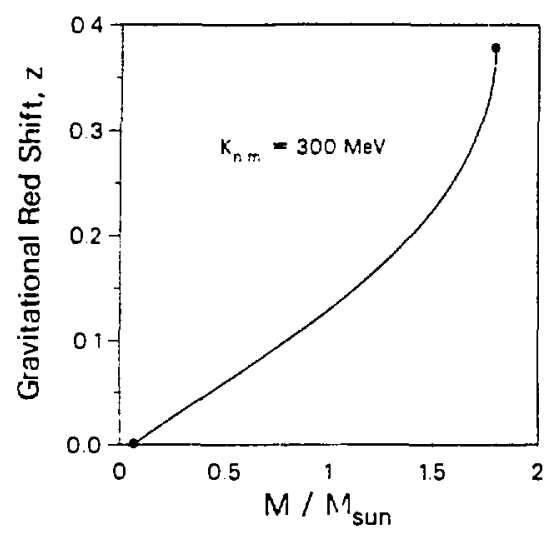

Figure 28: Surface gravitational redshift as a function of neutron star mass. [41]

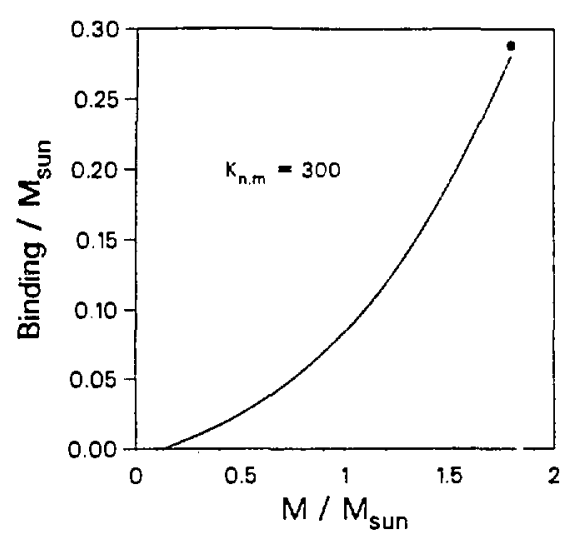

Figure 29: Gravitational binding energy as a function of neutron star mass. [41]

In Figs. 29 and 30 we show the gravitational binding and baryon number of neutron stars as a functicn of their mass, and in Fig. 31, the fraction of baryons that are strange is shown. This attains a value of $20-30 \%$ for the star at the limiting mass. This star, with central density $\rho_{c} \approx 7.2 \rho_{0}$, is dominated by hyperons in the central core, as can be inferred from Fig. 22. The loss of binding at the lower limit of the range of neutron stars, which occurs for $M \approx 0.069 M_{\odot}$ and a central density of $\rho \approx 0.46 \rho_{0}$, and the rapid growth in radius as this limit is approached from above, correspond to each other. At the upper range of masses, the rapidly declining radius, and increasing redshift, binding and strangeness fraction are all precursors of the gravitational collapse to a black hole, as the mass approaches the limiting mass. This occurs for a central dens:ty $\rho_{c} \approx 7.2 \rho_{0}$, and the corresponding mass is $M \approx 1.8 M_{\odot}$.

\section{Summary of Constraints on the Equation of state}

We have reviewed the prospects for obtaining constraints on the equation of state from astrophysical sources. Neutron star masses although few are known at present, a situation which may soor. change as remarked earlier, provide a very direct constraint in as much as the connection to the equation of state involves only the assumption that Einstein's general theory of relativity is correct at the macroscopic scale. This 


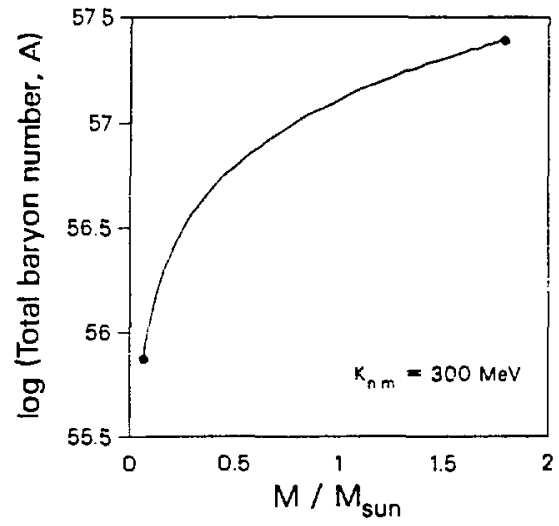

Figure 30: Baryon number of neutron stars as a function of mass.

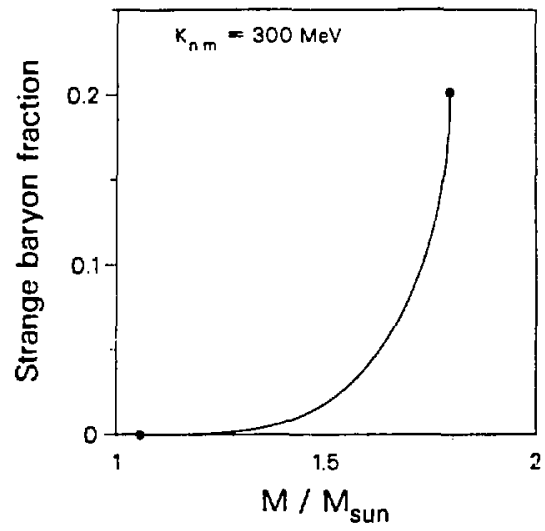

Figure 31: Fraction of baryons that are strange as a function of neutron star mas. [41]

is almost as secure as Maxwell's equations. There are nevertheless a number of uncertainties, prime among them, as concerns extracting the compression modulus, are the nyperon couplings. Nevertheless what is abundantly clear is that aily model that omits their presence in the star will underestimate the stiffness of the equation of state. Supernovae simulations involve such a plethora of physical processes including those involved in the evolution of the precollapse configuration, not all of them known or understood, that they provide no constraint at the present time. Not even the broad category of mechanism for the explosion has been agreed upon (prompt shock, delayed shock) until recently. In Fig. 32 we summarize our findings from a number of sources of information on the compression modulus [35].

We evaluated the vacuum polarization effects on the equation of state and neutron stars in the $\sigma, \omega, \rho$ theory. These effects are not negligible, although they are considerably smaller than found for the chiral sigma model $[64,78]$. However it was found that when the coupling constants are renormalized so as to reproduce the five saturation properties of nuclear matter in each case, whether or not renormalization is carried out, the equation of state and neutron star properties are virtually identical in the two approximations. On the other hand, when only the saturation density and binding are controlled as in ref.[63], the equation of state and neutron star properties, computed with and without vacuum polarization diverge at higher density. Failure to adequately constrain the equation of state at saturation can therefore lead to spurious conclusions in applications to dense matter as in neutron stars, as well perhaps in 


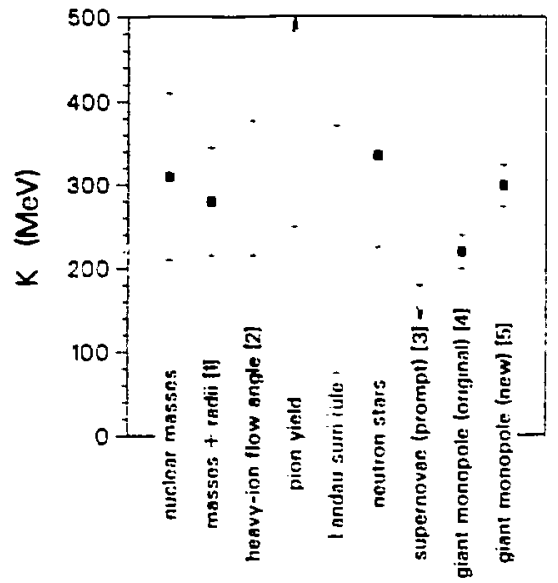

Figure 32: Summary of results on $K$ see reference for details. [35]

applications to nuclear structure, especially for properties that depend on $K$ or $m^{\star}$.

The question of the sensitivity of neutron star masses to the saturation properties of the corresponding nuclear matter was studied. First, as discussed above, whether the $\sigma, \omega, \rho$ theory yields the same or different equation of state at high density when vacuum polarization effects are incorporated, depends on how tightly the saturation properties are controlled. So within this theory, which is the only known relativistically covariant field theory of matter that can account for both nuclear matter and finite nuclei, the saturation properties and the higher density behavior are intimately connected. In fact this has to be true of any comprehensive theory of matter, since the coupling constants everywhere specify the equation of state. It need not be true of parameterizations of the equation of $s^{t}$ ate, for which there is no underlying theory. Second, we explicitly demonstrated, that although the density of matter at the center of a neutron star at the mass limit is fairly high, $\rho_{c} \approx 7 \rho_{0}$, the mass of a star even there, is not dominated by dense matter. Instead, fully one half is contributed by matter at densities less than three times nuclear density. This also establishes a dependance of the limiting star mass on the equation of state near saturaticn.

We calculated a number of additional neutron star properties, that may become tests of the theory as more data on neutron stars becomes available. We reemphasized the role of equilibrium in neutron star structure and the equation of state. The fraction of baryons that are hyperons in the limiting mass star is about 20 percent, and hyperons are the dominant baryons in the central core. 

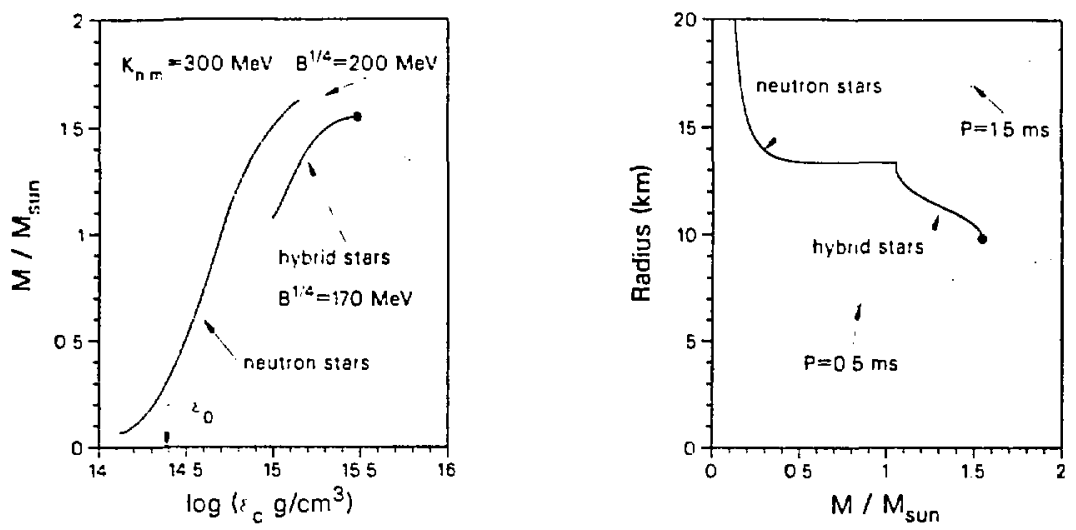

Figure 33: Generic relations for hybrids are the same as for neutron stars as Fig. 6, but hybrid stars may have a first order phase transition between hadron and quark phases (the region of (nearly) constant $\boldsymbol{M}$ ). Dotted regions are unstable. Stable regions marked by solid lines. [77]

\section{Stars with Quark Cores: Hybrids}

In earlier sections we have studied quark matter, neutron star matter and nuclear matter, and have discussed phase transitions between the confined and deconfined states. Such transitions may be induced in relatjvistic collisions between nuclej because of the high density and temperature produced. The phase transition from the deconfined to confined phase almost certainly took place in the early universe. It may also take place in the cores of the more massive neutron stars [79]. Such stars, I call hybrid. (Possibly the first calculation of the structure of a star for which a first order phase transition of unspecified nature occurs was made by $\mathrm{C}$. K. Chung and T. Kodama, H.ev. Bras. Fis. 8 (1978) 404.)

Usually it is (tacitly) assumed that hadronic matter, in which quarks are confined in nucleons as in the nuclei of which the world around us is made, is the absolute ground state of the st rong interactions. In this case such hadronic matter can coexist with cuark matter at sufficient pressure, but if the pressure is released, that matter will ret urn to the hadronic state. If the pressure due to gravity is sufficiently high in the core of a compact star we expect that it will convert to quark matter so that the star has a quark core and a neutron star exterior, and the whole would be bound by gravity.

We have learned the techniques of dealing with phase transitions, and do not 
need to further discuss them. Instead We show in Fig. 33 some of the features that arise in this case. The less massive stars will of course be purely neutron stars, in the sense already described (equilibrium mixture of nucleons, hyperons and leptons). The most massive ones will, if the phase transition occurs at low enough densities to be realized in the star, have a core made of $u, d, s$ quarks. It is easy to show that already at moderate densities the Fermi energy of the u,d, quarks exceeds the mass of the s-quark, so that it is also populated. In the mass vs. central density plot, two possibilities are shown. In the one $\left(B^{1 / 4}=170 \mathrm{MeV}\right)$ the mixed phase occurs in the density range of about $\log \epsilon_{c}=14.7$ to $15\left(\epsilon_{c} / \epsilon_{0} \approx 2\right.$ to 4$)$. Stars in this range of central densitics are unstable, or only marginally stable. Below this region the stars of the sequence are pure neutron stars; above it they are hybrids with quark cores and a neutron star mantle. In the second case $\left(B^{1 / 4}=200 \mathrm{MeV}\right)$ the pure neutron stars have central densities as high as $\log \epsilon_{\mathfrak{c}}=15.1\left(\epsilon_{c} \approx 5 \epsilon_{0}\right)$. Stars with central densities falling in the mixed and quark phase above this density $O$ f course the values of the bag constant for which these behaviors occur depends on the nuclear equation of state which here has $K=300 \mathrm{MeV}$ and $m_{\text {sat. }}^{*}=0.8 \mathrm{~m}$. In this case, for $B^{1 / 4}$ a little larger than $200 \mathrm{MeV}$, the central density in the star sequence never attains a high enough value to reach the phase transition.

In the two examples discussed, the maximum mass of the sequence of stars is above $1.5 M_{\odot}$, in the one case a pure neutron star and in the other a hybrid. For intermediate values of $B^{1 / 4}$ the limiting mass becomes smaller and between 180 and $190 \mathrm{MeV}$ has a value of about $1.4 M_{\odot}$ and switches from being a hybrid star (at $B^{1 / 4}=180 \mathrm{MeV}$ with mass $1.35 M_{\odot}$ ) to a pure neutron $\operatorname{star}\left(\right.$ at $B^{1 / 4}=190 \mathrm{MeV}$ with mass $1.48 M_{\odot}$ ). The macroscopic properties of these compact objects, their masses and radii, are very similar. Whet her their surface properties are sufficiently different as to allow a distinction between them has not so far been investigated. It is conceivable for example that star quakes and microquakes may distinguisl between them since these phenomena may be related to seismic activity associated with changing stresses as the star's rotational frequency diminishes and the stress is released in a quake which is signaled by a sudden small change in the period (fig. 2). It may be possible therefore to gain information on the phase transition in cold equilibriated matter from compact stars.

As with neutron stars, the window in $M$, and hence in $A \sim M / m$, for which fast rotation can be sustained is very narrow, which is inevitable since both types of stars are bound by gravity. This is seen in the second part of the figure.

In Fig. 34 we compare the density profile of neutron and hybrid stars and a star we will discuss in detail, and have alluded to earlier, a strange star, one that would exist if strange matter were the absolute ground state. 


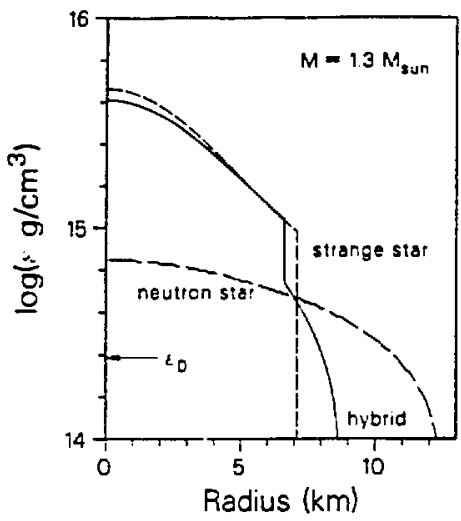

Figure 3: Density profiles of neutron and hybrid stars and a hypothetical star of strange quark matter under the assumption that strange matter is absolutely bound. [80]

\section{Fast Rotation of Pulsars and the Equation of state}

Several years ago it was believed that a very fast pulsar had been discovered in the remnant of the recently discovered supernova 1987A. This stirred great interest in the implications such fast rotation as 2000 times per second might imply for the equation of state of matter. One obvious problem is that at some point centrifuge will overwhelm gravity. This places an absolute upper bound on rotation (see eq.(25). The discovery has later been withdrawn. However I have emphasized in my work, and will discuss it in greater detail later, that there are search biases against the detection of pulsars below about a millisecond. I discuss the reason latter, and it is presented in detail in ref. [81]. Essentially you can think of it this way. In making a survey of the heavens for pulsars you can spend all your time and computer resources in aiming the radio telescope at one direction in the sky, and be very certain that if there is a pulsar there you will find it. You will have wasted all your rescurces if there is not one in that direction. Hence the first compromise decision that must be made. For the moment I recall that the fastest known pulsar has a period of $1.558 \mathrm{~ms}$ [82] with celestial coordinates $1937+21$. Since then a total of ten millisecond pulsars have been found. They have been found right down to the effective limit of sensitivity!

Einstein's equations for the structure of a rotating star are much more involved than the $\mathrm{O}-\mathrm{V}$ equations that we have been studying. As far as the absolute upper limit on the frequency is concerned (called the Kepler frequency), it can be approximated by eq. (25) with the empirical factor cited. It approximates the values obtained 
by exact numerical integration of the equations of rotating star structure very well $[9,83]$. We have shown in some of the Figures $(6,33)$ the boundary in the massradius relation for which stars can rotate rapidly. It was evident that none of our models could sustain rotation as fast as $1 / 2 \mathrm{~ms}$. Some neutron star models can do so [9], but as we pointed out [8.1] and reconfirmed [50], the central density is so high $\left(\epsilon_{c}>12 \epsilon_{0}\right)$ as to call into serious question whether the star can be composed of individual hadrons. From geometrical considerations alone, the baryon density for cubic close packing of classical spheres of radius equal to the charge radius of protons is $1.6 \rho_{0}$. If packed to the 'hard core radius' say $0.5 \mathrm{fm}$, the factor is 6.7 . And this does not reflect the effects of the uncertainty principle which would give nucleons enormous momenta with which to destroy each other's structure if packed to such densities. Very fast pulsars a $\_$not likely to be neutron stars or hybrids. This raises an issue of fundamenial significance which we discuss in the next section.

To make matters worse, our discussion above is in terms of the Kepler frequency. It has been known for some time lowever that the limit on rotation is not in practice set by the hepler frequency: it is only an absolute upper limit. In practice the limit is set by gravitational radiation reaction instabilities, that are especially active in hot stars, and all neutron stars are hot when born (see ref. [85]). Therefore if one is born with very rapid rotation, say caused by the spin-up due to angular momentum conservation of an especially rapidly rotating progenitor, it will rapidly spin down by radiating gravitational waves until as a result of its cooling these instabilities are damped by viscosity which increases as $1 / T^{2}$. At that time the pulsar is no longer the rapid rotor it started as.

However the full import of this instability las not been appreciated until we investigated its effects on modern theories of dense matter $[86,87]$. There we found that the effective limiting frequency is only $60-70 \%$ of the Kepler frequency. This effectively limits the possibility of understanding a neutron star as a fast pulsar only for periods greater than about $1 \mathrm{~ms}$. While the theoretical analysis is beyond the scope of these lectures, it is important to know the result.

\section{Fast Pulsars, Strange Stars}

There is presently no evidence to support the commonly held assumption, apparently anthropocentric, that the confined hadronic phase of individual nucleons and nuclei is the absolute ground state of the strong interaction. The fact that most of the mass in every object that we know, from our own bodies to all the visible galaxies resides in nucleons and nuclei tells us for certain only that this is a possible phase of matter and that it is very long lived; not necessarily that it is the lowest energy one. How misleading the present composition is in revealing the nature of the ground state is immediately exposed by noting that the lowest criergy state of the confined phase is $\mathrm{Fe}^{56}$ and there is very little of that in the universe. From the QCD energy scale it is quite plausible that the deconfined (3-flavor) strange-quark-matter phase is 
lower in energy $[20,21]$, and we show that the present upper limit on the abundance of strange nuggets in the earth's crust does not rule out this possibility. Indeed the universe would be almost identical in either case. We are only just now entering an era in which advances in technology may allow the detection of the necessarily subtle signals that might be present if the universe exists in a metastable phase, albeit longlived, instead of the ground state. The most promising signals, both from the point of view of prospects for their existence as well as for their detection, are submillisecond pulsars. The shorter the pcriod of rotation the more secure the conclusion that the universe is in a metastable phase of matter[88, 84].

Of course the assumption that the confined hadronic phase is the ground state cannot be realistically challenged by resort to specific models of the equation of state of dense nuclear matter, nor of quark matter, but rather by use of model independent limits, and when it is necessary to invoke models of matter, by exploration of the most general forms subject only to the minimal generally accepted constraints. Our approach within this framework is to exhibit the difficulties and contradiction encountered in trying to understand very rapid rotation of pulsars if they are assumed to be neutron stars. We then show how these are naturally resolved under the assumption that strange matter is the absolute ground state.

\subsection{Bias against short periods of present pulsar surveys.}

As of this writing there are ten known millisecond pulsars with periods ranging between $1.6 \mathrm{~ms}$ to $7.9 \mathrm{~ms}$, all of them discovered recently, and of course 400 others, with a mean period of $700 \mathrm{~ms}$ and a maximum period of $4 \mathrm{~s}$. What is not generally realized however is that searches for radio pulsars are biased, being least sensitive to short periods[81]. The bias exists because of compromises involving choices of sampling rates of the radio signal, number of frequency channels, the sophistication of the algorithm for correcting the data because of the differential dispersion of the radio frequencies in the bandwidth of the receiver caused by the unknown column height of the interstellar plasma, the number of iterations over such corrections and the Fourier analysis of the corrected data that has to be carried out at each stage. Therefore the world's data on radio pulsars does not represent the underlying population because of this search bias against detection of short periods, especially below $1 \mathrm{~ms}[89]$, and most of the large surveys have had no sensitivity below about $4 \mathrm{~ms}$ as emphasized by[81]. And empirically fast pulsars are seen only at radio frequencies. The cutoff in short periods that appears in Fig. 35 is therefore possibly only an artifact of the search sensitivity. The growing number of pulsar discoveries with periods right down to the cutoff suggests that this is so and presents a special opportunity and challenge to radio astronomy! For while the discovery of additional millisecond pulsars is exciting, both because of their novelty and for what they may reveal of the evolutionary processes involved in their creation and the environment of galactic globular clusters, which are now understood to provide an environment that is especially favorable for 
the incubation of fast pulsars[90], the discovery of a single sub-millisecond pulsar, say below $1 / 2 \mathrm{~ms}$, addresses the fundamental issue of the ground state, and would provide strong if not conclusive evidence that we inhabit a metastable phase.

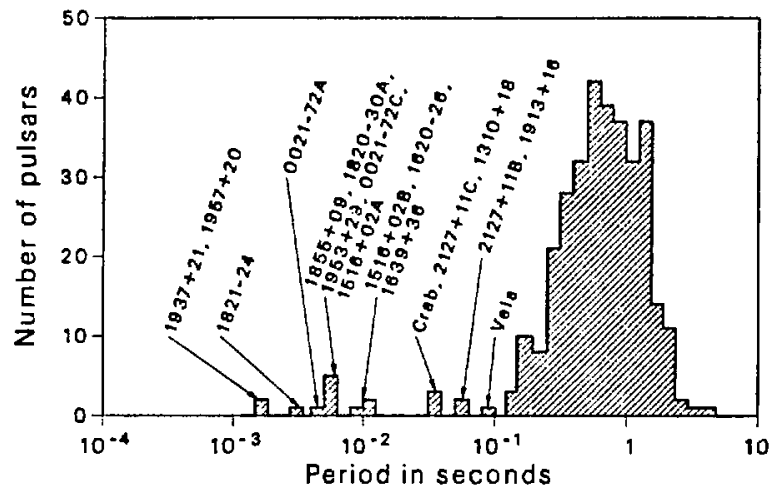

Figure 35: Distribution of pulsar periods. There is a relatively strong attenuation in sensitivity of radio pulsar surveys for periods below about $1 \mathrm{~ms}$. Pulsars are identified by their celestial coordinates.

\subsection{Difficulty in reconciling fast pulsars with neutron stars.}

The relevance of fast pulsars is easily understood in terms of their stability against mass loss, expressed as the dominance of gravity over centrifuge (see eq.(25),

$$
\Omega<\Omega_{K} \approx \alpha \sqrt{G M / R^{3}},
$$

where $\Omega$ is the angular velocity, and $\alpha \approx 0.65$ is an empirical GR correction to Newtonian physics[9]. This places a model independent constraint on the average energy density of the star. In actual stars the density increases monotonically toward the center. To discover the implication of fast rotation on the central density of the star, we must solve Einstein's equations for star structure using computed or hypothetical equations of state. For very high central densities, it is expected that matter consisting of individual nucleons will dissolve into quark matter, of which strange quark matter, an approximately equal mixture of $u, d, s$ quarks, is lower in energy than nonstrange 2-flavor quark matter. The problem resolves itself into understanding at what rotational period, stability requires a central density that exceeds the phase transition density. Three questions arise at this point: (1) Can a compact star rotate very 
rapidly and with central density that is plausibly below the phase transition point so that it is a neutron star? (2) Can a hybrid star, a neutron star with a quark core, rotate very rapidly? (3) What is the minimum rotational period in either case? One way of attempting to answer these questions is by examining the properties of stars based on specific theories of dense nuclear matter. The drawback of this approach is that we do not have an impeccable theory of dense matter, and we certainly cannot solve QCD to obtain an equation of state. We cannot be assured therefore that of a finite collection of specific theories we have spanned the characteristics of the equation of state of nature. Our approach is therefore to carry out an exhaustive grided search, similar to our earlier one $[77,80]$, over a flexible parameterization of the equation of state that is constrained only by causaiity, its smooth matching to the sub-nuclear equation of state $[91,12]$ and the requirement that the maximum mass neutron star be at least $1.44 M_{\odot}$. The result is summarized in Fig. 36, of a search over more that 1400 models of the equation of state spanning a broad spectrum of behavior from soft to stiff at low density and independently at high, and including first or second order phase transitions representing pion or kaon condensates, for example, subject only to the above minimal constraints. The least central density required by the condition of stability to rotation is shown as a function of rotation period. Particular theories of nuclear matter can yield results that lie above or on this curve, but not below! So as to be conservative, we have included the effect of gravitational radiation-reaction instabilities as a reduction in the Kepler frequency, $\Omega_{G R}=\beta \Omega_{K}$, at the minimum estimate, $\beta=0.91$ [85]. The correction may be much larger, like $\beta \approx 0.7$, for realistic neutron star models[87]. Two important conclusions can be reached. Pulsars with periods longer than $1 \mathrm{~ms}$ can be understood as neutron stars having modest central densities as low as three times nuclear density. In contrast, pulsars with periods shorter than $1 / 2 \mathrm{~ms}$ must have very high central densities if they are bound only by gravity, as is the case for neutron stars since neutron matter is unbound, and so is nuclear matter above about $A=250$. For such short periods the density must be so high as to render implausible the contention that the star is composed of individual nucleons. We do not know from experiment at what density the expected phase transition to quark matter occurs, and we have no guide yet from lattice QCD simulations. But from geometric considerations it is implausible that nucleons survive at densities above a few times nuclear density[84].

The minimum rotation period found in the exhaustive search over equations of state was $0.42 \mathrm{~ms}$. The dotted curve in Fig. 36 is merely an extrapolation. It appears that no star bound only by gravity can have $P<0.4 \mathrm{~ms}$. These figures for the period correspond to the conservative estimate of GR instabilities. While we wish to be conservative in arriving at our conclusions, we also want to stress that for realistic equations of state the GR instabilities increase the scale of the period axis in Fig. 36 by about a factor $1.3[86,87]$. So it is doubtful that any phenomenological neutron star model can have a stable period as low as $0.55 \mathrm{~ms}$, and from our study of neutron stars computed from realistic equations of state calculated in relativistic 


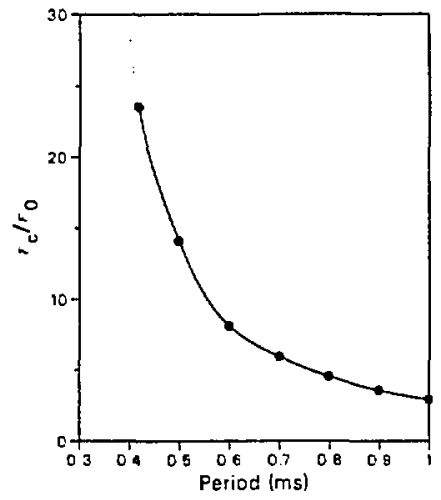

Figure 36: Least central density of 'neutron' star as a function of shortest stable rotation period. Each dot represents a 'neutron' star whose equation of state satisfies constraints described in the text and has least possjble central density, $\epsilon_{c}$, consistent with stability down to the rotational period shown, including GR instabilities.

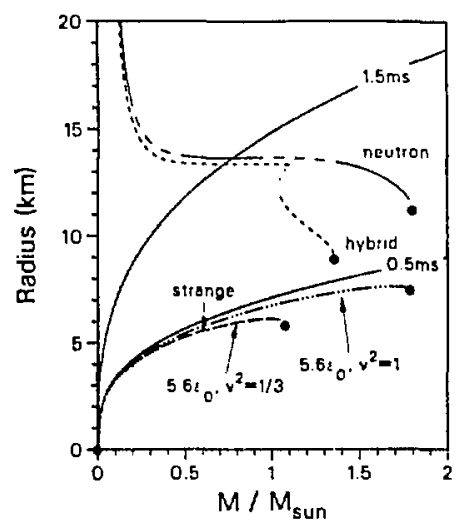

Figure ?'i: Generic relations for cor:pact stars bound by only by gravity (neutron and hybrid) and stars mose of self-bound matter (strar

below solid lines can rotate $a . \cdots:$ than the indicated period. Equation of state for strange matter parameterized as $t=p / v^{2}+\epsilon_{b}$, and star families labeled by value of $\epsilon_{b}$ and $v^{2}$.

Hartree-Fock approximation including two-particle correlations and based in one case on the well known Bonn meson-exchange potential that reproduces scattering data so well, it is doubtful that neutron stars can rotate with periods below about $1 \mathrm{~ms}$ $[87]$.

For compact stars that are bound only by gravity (in contrast to hypothetical stars made of self-bound matter, as may be the case for strange quark matter), the mass radius relationship has a characteristic form shown in Fig. 37. For low masses, where the gravitational attriction is weak, the star is large. For higher masses, near the limit for collapse to a black hole, the radius decreases very rapidly with increasing mass. For such stars, only those very close to the mass limit can rotate rapidly because there the radius is least and the mass greatest (see Eq.(89)). In other words, a very rapidly rotating neutron (or hybrid star) must be very finely tuned in mass, or equivalently baryon number. Therefore if two very fast pulsars of about the same period but different masses are found, both cannot be a star bound only by gravity, that is to say a neutron or hybrid star.

If very rapidly rotating pulsars cannot be reconciled with neutron stars the 
least drastic hypothesis that can be considered next if pulsars with periods in the range $0.4 \mathrm{~ms} \leq P<1 \mathrm{~ms}$ are discovered, is that they are neutron stars with quark cores, what we have called hybrid stars[77]. There is no natural limit on their central density. In their cores the nucleons are dissolved into quarks by the high pressure induced by gravity. Such stars could exist if the confined phase is the ground state; they are bound only by gravity and consequently the radius-mass relation is of the generic form shown in Fig. 37 just discussed. However, there are theoretical grounds to doubt that hybrid stars are plausible candidates for fast pulsars, but no proof at this time[80, 83]. Very briefly, the attributes of an equation of state that would allow a star bound only by gravity to have stable rapid rotation is that it be soft at low and intermediate density, and very stiff at high, at or near the causal limit. The last requirement seems incompatible with the notion of asymptotic freedom in the deconfined phase. Moreover in the exhaustive search reported earlier [80], we found that hybrid stars, with the necessary restriction that the mixed phase has its lowest density above nuclear saturation, cannot rotate faster than the fastest neutron star models and then only with the stiffness just spoken of that seems incompatible with the notion of asymptotic freedom.

In view of the difficulties of reconciling a neutron or hybrid star with fast rotation, the implausibly high density for neutron matter, the stiffness at high density required of the equation of state that seems incompatible with quark matter, the fine tuning in mass of the star so as to place it close to the termination point, all of which arise in trying to reconcile a star that is bound only by gravity with fast rotation, an altogether different possibility needs to be examined.

\subsection{Strange matter as the absolute ground siate.}

Witten[20] hypothesized that strange quark matter consisting of an approximately equal mixture of $u, d, s$ quarks is absolutely stable, as a solution to the so-called missing mass or dark matter problem. It is believed by some astronomers, based both on cosmological arguments (closure of the universe) and on the observations that the luminous matter of the universe is apparently subject to greater gravitational forces than the luminous matter itself would exert, that there is much more matter in the universe, indeed within our own galaxy, than can be seen. Sucll matter is referred to as dark matter, and has been the subject of much speculation. Witten reasoned that since the universe in the early high temperature era almost certainly passed through the deconfined quark matter phase, of which three-flavor quark matter is lower in energy than two flavor, then if strange matter were the ground state, much matter would have remained in this phase. If it is the ground state, then objects from microscopic to star-like quantities would be stable, since it is self-bound. Such matter in microscopic lumps called strange nuggets would be non-luminous and therefore invisible as objects in space. Hence the suggestion that it formed the dark matter of the universe. However it was very soon shown that even if it is the ground state, 
strange matter would have evaporated in the high temperature era into ordinary hadrons [92], and there would be no relic strange matter. So strange matter as a candidate for dark matter was not a viable suggestion. However, it may still be true that it is the ground state, and we discuss how this is both plausible and violates no known facts.

We know that non-strange quark matter is higher in energy per baryon than nuclear matter, otherwise nuclei would decay promptly into it. It must be higher by about the QCD energy scale, $\sim 100 \mathrm{MeV}$, established by lattice simulations. As a rough estimate treat the quarks as a Fermi gas. The energy density scales as $\epsilon \sim \gamma \mu^{4}$ and the baryon density as $\rho \sim \gamma \mu^{3}$, where $\gamma$ is the degeneracy, 2 or 3 . Comparing the two types of quark matter at the same baryon density, then $\mu \sim \gamma^{-1 / 3}$ so that 3 -flavor quark matter has an energy per baryon, $t / \rho$, of about a factor $(2 / 3)^{1 / 3} \sim 0.9$ times that of 2 -flavor, or about $100 \mathrm{MeV}$ lower. That places 3-flavor quark matter at about the same energy as nuclear matter. However, even if strange matter is lower in energy, ordinary nuclei can decay into the strange quark phase phase only on a time scale long in comparison with the age of the universe, since it is inhibited by the need for $A$ simultaneous strangeness changing weak interactions where $A$ is the atomic number[21]. This is because it is energetically unfavorable for the transition to take place one quark at a time since this would only produce hypernuclei. It is important in the above argument to realize that surface and shell effects place a lower but uncertain bound $(A=10-1000)$ on the baryon number for which small nuggets of strange matter are bound even if strange matter in bulk is absolutely stable[21].

Althogh the mass of the strange quark is larger than that of the $u$ and $d$ quarks, at any density above several times nuclear, the quark chemical potential exceeds the mass difference, and u,d,s quarks are almost equally populous. However because of the mass difference there is a slight deficit of strange quarks, and this provides a nugget with a positive charge. This insures that the nugget will repel nuclej, and so will be inert except in a neutron rich environment such as neutron stars or the late stage of star collapse when the density exceeds the neutron drip density, or in very high temperature matter where the kinetic energy of charged particles may overcome the Coulomb barrier. The anomalously low charge to mass ratio $Z / A$ provides a signature for their identification if they occur on earth with sufficient concentration to be found. However as we shall see, the concentration expected on earth is very small. We will discuss this later. Because of their high density, a few times nuclear density, the surface gravitational force acting on nuggets with $A>10^{17}$ would exceed the electrostatic forces holding solid material together so that they would sink toward the center of the earth[21].

\subsection{Strange quark stars.}

If strange-quark matter is self-bound and absolutely stable as as has been suggested[20, 93], the structure of compact stars made of it would be entirely different from that 
discussed above[20, 94, 95]. For strange stars gravity merely prevents them from fissioning into smaller bodies, and of course imposes a mass limit: they are otherwise bound by the strong interaction. Denote the normal energy density of such matter, the density at which the internal pressure vanishes, by $\epsilon_{b}$. A small nugget therefore has mass $M=\frac{1}{3} \pi R^{3} c_{b}$, so that, unlike neutron stars, or more generally stars bound only by gravity, the mass radius relation for small mass is $R \propto M^{1 / 3}$, and has a generically different form as shown in Fig. 37. Therefore the entire family of strange stars can rotate rapidly, not just those near the limit of collapse to a black hole[84], since for such a radius-mass relation the expression for the Kepler frequency, Eq.(89), is a constant for low mass stars and changes for those near the mass limit only in the sense of increasing. Also from Eq.(89) we can derive a condition on the normal density of self-bound stable matter that will allow all such stars to rotate with period $P:[80]$

$$
\epsilon_{b} \geq \frac{3}{4 \pi G}\left(\frac{\Omega}{\alpha}\right)^{2}=1.3 \epsilon_{0}\left(\frac{\mathrm{ms}}{\mathrm{P}}\right)^{2} .
$$

For example, all strange stars can rotate with $P=1 / 2 \mathrm{~ms}$ if $\epsilon_{b}>5.2 \epsilon_{0}$ (where $\epsilon_{0}=$ $2.5 \times 10^{14} \mathrm{~g} / \mathrm{cm}^{3}$ is normal nuclear density). Gravitation radiation-reaction instabilities are unimportant for quark stars because of the expected high viscosity[18], so the correction $\beta$ quoted earlier for neutron stars does not need to be applied. Thus three problems are solved if strange quark matter is the ground state; very short periods are possible, below $0.4 \mathrm{~ms}$, provided only that the normal density satisfies the above model independent relation, high central densities as required by fast rotation are natural for quark matter and a st. does not have to be finely tuned in mass to be stable at very fast rotation.

Based on the bag model of confinement and the range placed on its parameters by the condition that strange quark matter is the ground state, several authors have asserted that strange stars cannot rotate very rapidly[83, 96, 97] or can do so only marginally[98]. There is an unfortunate confusion in logic there in translating a statement that is true of a crude model to a statement about nature. We have emphasized elsewhere that whether or not strange stars can rotate very rapidly is entirely an experimental question, not a theoretical one[84]. For to determine on theoretical grounds whether the energy per nucleon in strange matter lies below that in $\mathrm{Fe}^{56}$ with $E / A=930 \mathrm{MeV}$, which would make it absolutcly stable, or lies above the nucleon mass at $939 \mathrm{MeV}$, would require one percent accuracy, an accuracy certainly not possessed by the bag model, nor indeed by lattice QCD now or in the foreseeable future. Neither theory nor confinement models can rule out the hypothesis that strange matter is stable, nor conversely can they be used to assert that it is.

If the hypothesis is true, then some or all pulsars may be strange stars rather than neutron stars. Strange stars can be produced in at least two ways $[99,100]$. If the density in the core of the more massive neutron stars exceeds the transition density to quark matter, the core will spontaneously convert to non-strange quark 
matter. In turn, this will weak decay to strange matter, and then conversion of the entire star will occur on a short time scale. If a nugget of strange matter falls into a star, jt will gravitate to the center and lie dormant until the star collapses. When the density exceeds the neutron drip density, the nugget will begin to grow, and convert the entire neutron star produced in the supernova. It is possible that the universe is sufficiently contaminated by strange nuggets, whose abundance is discussed in the next section, that the second process will always preempt the first.

\subsection{Strange matter compatible with terrestrial searches.}

If strange stars exist, there will be present on earth some abundance of strange nuggets accumulated as cosmic rays over the earth's lifetime. We now estimate an upper limit to see if strange matter as the ground state is ruled out by present experimental limits. From 16 years of observations on the binary pair of compact stars with celestial coordinates $1913+16$, the decay of the binary orbit has confirmed Einstein's gravitational radiation to an accuracy of less than one percent[5]. Eventually the decay of this orbit will cause the compact stars to collide, and some fraction of their material will be injected into the galaxy. The decay time is short compared to the galactic age. In our estimate of the abundance of such material that has accumulated on earth, we employ accepted cosmological figures for those items that are avajlable, and overestimates of others, so as to get an upper bound on the concentration of strange nuggets. For this purpose we need the age of the galaxy $\left(10^{10}\right.$ years $)$, the frequency of type II supernovae $(1 /(100 \mathrm{yr}))[10 !]$, the fraction of pulsars that occur in binary compacts $(<1 / 100)$, the fraction of mass ejected from a collision $(1 / 10)$ [102]. We find a mass density of debris from compact star collisions of $<10^{-29} \mathrm{~g} / \mathrm{cm}^{3}$. (For the volume of the galaxy in which the ejecta is contained we take $\pi \times 8^{2} \times 2 \mathrm{kpc}^{3}$ ). As an extreme overestimate, assume that all such compacts were strange and that all mass is ejected in minimum mass fragments $(A \approx 1000[21])$. We find a number density of strange nuggets of $<10^{-8} / \mathrm{cm}^{3}$. Assuming a typical galactic velocity of $10^{7} \mathrm{~cm} / \mathrm{s}$, there would be an influx of $<10^{15} / \mathrm{cm}^{2}$ over the age of the earth $\left(5 \times 10^{9}\right.$ yr). (However, the velocity assumed corresponds to the measured typical velocity of pulsars transverse to the galactic plane[103]. If the isotropic component is less, then our flux is an overestimate.) The earth's crust is not tranquil. Mountains are cast up and then eroded, continents drift, tectonic plates collide, slide one over the other. Material that can be recognized as once having been at the earth's surface, resurfaces in the lava of volcanic eruptions, having been subducted to the molten core below the mantle[104]. Estimating a geologic mixing denth of the order ten kilometers, a very conservative one, then even if all such nuggets were stopped at the surface, they would be diluted to a concentration of $<10^{10} / \mathrm{cm}^{3}$ or less over geologic times, or $<<10^{-14}$ nuggets per nucleon given an earth density of $5 \mathrm{~g} / \mathrm{cm}^{3}$. This extreme overestimate is to be compared with the upper limit established by experiment of $10^{-14}$ [105]. So the hypothesis cannot be ruled out by present experimental limits on the abundance 
of strange nuggets in the earth's crust! There are probably no favorable samples on earth for strange nugget searches, in view of the high degree of dilution. Meteorites have their surfaces burned off in traversing the atmosphere if they are not completely destroyed, and so also are not favorable. However the moon's surface has been exposed to the hypothetical strange cosmic rays without geologic mixing, and with only minor meteoritic disturbance of its surface since very early times, and so moon rock might prove to be a favorable sample for mass spectroscopy (because of the anomalously small $Z / A$ of strange matter) or for a Coulomb back scattering experiment such as that of ref.[105].

It has been understood for several years that even if strange matter is the lowest energy state when cold, and that the early universe passed through this phase, it was so hot at the time that strange matter would have evaporated into hadrons $[99,92]$. Little if any primordial material is expected to have survived. For these reasons and those developed in the preceding paragraphs we understand that the universe would have evolved along essentially the same path and aside from very subtle signals would appear the same now, no matter which is the ground state. Only at the death of massive stars when dense matter that is cold on the nuclear scale is produced in the resulting neutron stars, may conditions for the creation of cold strange matter occur for the first time. Whether such conditions have ever been achieved depends on the unknown phase transition density, and whether it has been reached in the core of any neutron star.

\subsection{Motivation for submillisecond pulsar searches.}

It is not possible to prove that the ordinary confined hadronic phase of nucleons and nuclei is the ground state. It can only be disproved with the discovery of a lower one. As remarked, the QCD energy scale makes strange matter an equally plausible ground state. A strong indication that it is so would be the discovery of submillisecond pulsars, especially several of different masses. While mass measurements are scarce, we note that the recent discoveries in globular clusters and the expected high population of fast binary pulsars in them may change this situation dramatically. It could of course be a coincidence that the lower limit on the period sensitivity of radio pulsar surveys, resulting from the particular compromises chosen in present day searches, matches the actual cutoff in pulsar periods. But the accelerating discovery rate of millisecond pulsars with periods right down to the limit of present day sensitivity suggests the tantalizing prospect that there are even faster ones. Improvement of the sensitivity is not beyond present technology and as physicists and astronomers we may learn as a result something that we do not know but ought to, the structure of the true ground state of the strong interaction. 


\subsection{Accelerator searches for strange nuggets.}

Having motivated, hopefully, a search for submillisecond pulsars, we wish to affirm the importance of accelerator based attempts $[100,10 \%, 108]$ to produce strange nuggets in relativistic nuclear collisions $[109,110]$. These are long-shot experiments: the dense matter produced for $\approx 10^{-22} \mathrm{~s}$ is hot and has no time to develop a net strangeness. Strange nuggeis. if strange matter is stable, could be produced only as the result of two types of simultaneous fluctuations that separate strange and anti-st range quarks, and that also cool the nugget so that it does not evaporate. Moreover the nunber of quarks accessible to nuclear collisions may be too small to defeat the finite number destabilizing effects[21]. Cross sections in any case will be very small. But the production and capture of a strange nugget, besides being incontrovertible evidence, may prove important as a compact energy source[i11]. So while the astronomical prospects for discovery of strange natter appear better to us, the practical consequences of laboratory production are potentially enormous.

Acknowledgements: I appreciate discussions with D. C. Backer, Astronomy; UC at Berkeley: on pulsar searches and B. M. Smith, Earth Sciences Division, LBL, Berkeley, on geologic mixing of matcrial in the earth's crust. This work was supported by the Director. Office of Energy Research. Office of High Energy and Nuclear Physics, Division of Nuclear Physics, of the C.S. Department of Energy under Contract DEAC03-i6SF00098.

\section{Appendix}

\subsection{Stress-Energy}

The canonical stress-eriergy tensor that is derived from the invariance of the Lagrangian to infinitesimal displacements (see cg. [29]) is

$$
\mathcal{T}^{\mu \nu}=-g^{\mu \nu} \mathcal{L}+\sum_{\phi} \frac{\partial \mathcal{L}}{\partial\left(\partial_{\mu} \phi\right)} \partial^{\nu} \phi
$$

where the sum is over the various fields, $O$, in $\mathcal{L}$. For the Lagrangian of the real scalar field, say $\sigma$, corresponding to a chargeless meson, the Lagrangian has the well known form.

$$
\mathcal{L}=\frac{1}{2}\left(\partial_{\mu} \sigma \partial^{\mu} \sigma-m_{\sigma}^{2} \sigma^{2}\right)=\frac{1}{2}\left(g_{\mu \lambda} \partial^{\lambda} \sigma \partial^{\mu} \sigma-m_{\sigma}^{2} \sigma^{2}\right)
$$

where $\partial^{\mu}=\partial / \partial r_{\mu}$. In flat space. $g_{\mu \nu}$ is just the diagonal tensor,

$$
\left(\begin{array}{cccc}
1 & 0 & 0 & 0 \\
0 & -1 & 0 & 0 \\
0 & 0 & -1 & 0 \\
0 & 0 & 0 & -1
\end{array}\right)
$$


In general relativity it is neither diagonal nor constant under the most general circumstances. Notice that we have rewritten the usual first expression for $\mathcal{L}$ to display explicitly the metric. Now we can calculate $\mathcal{T}^{\mu \nu}$ from eq. (5). Do so and show that the result agrees with that obtained from the usual expression above.

\subsection{Schwarzschild Solution}

1. Consider a static star so that $g_{\mu \nu}$ are independent of time $\left(x^{0} \equiv t\right)$ and $g^{0 m}=0$. We choose spatial coordinates $x^{1}=r, x^{2}=\theta, x^{3}=\phi$. The most general form of the line clement is then,

$$
d \tau^{2}=U(r) d t^{2}-V(r) d r^{2}-W(r) r^{2}\left(d \theta^{2}+\sin ^{2} \theta d \phi^{2}\right)
$$

We may replace $r$ by any function of $r$ without disturbing the spherical symmetry: We do so in such a way that $W(r) \equiv 1$. Then we may write,

$$
d \tau^{2}=\epsilon^{2 \nu(r)} d t^{2}-e^{2 \lambda(r)} d r^{2}-r^{2} d \theta^{2}-r^{2} \sin ^{2} \theta d \phi^{2}
$$

where $\lambda, \nu$ are functions only of $r$. Compare with $d s^{2}=g_{\mu \nu} d x^{\mu} d x^{\nu}$ to read off,

$$
\begin{array}{r}
g_{00}=\epsilon^{2 \nu(r)}, \text { etc } \\
g_{\mu \nu}=g^{\mu \nu}=0, \quad(\mu \neq \nu)
\end{array}
$$

Hence from

$$
\begin{gathered}
g_{\mu \nu} g^{\nu \rho}= \begin{cases}1 & \text { for } \mu=\rho \\
0 & \text { for } \mu \neq \rho\end{cases} \\
g^{\mu \nu}=g^{\nu \mu}
\end{gathered}
$$

(repeated indices, one as a superscript, one as a subscript, are always summed unless otherwise stated) we have in this special case,

$$
g_{\mu \mu}=1 / g^{\mu \mu}
$$

So

$$
g^{00}=e^{-2 \nu(r)}, \text { etc }
$$

2. Recall the covariant $A_{\mu}$ and contravariant $A^{\mu}$ vector notation and the raising and lowering rules,

$$
A_{\mu}=g,{ }^{\mu \nu} \quad, \nu=g^{\nu \mu} A_{\mu}
$$


The Christoffel symbol is defined in terms of derivatives of the metric functions,

$$
\Gamma_{\mu \nu \sigma}=\frac{1}{2}\left(g_{\mu \nu, \sigma}+g_{\mu \sigma, \nu}-g_{\nu \sigma, \mu}\right)
$$

where, $\sigma$ means differentiation,

$$
g_{\mu \mu, o} \equiv \frac{\partial}{\partial x^{\circ}} g_{\mu \nu}
$$

It is frequently convenient to raise the first suffix on the Christoffel symbol,

$$
\Gamma_{\nu 0}^{\mu}=g^{\mu \lambda} \Gamma_{\lambda \nu 0}
$$

It is symmetric in the lower tho suffixes. In the case considered above, derive the non-ranishing Christoffel symbols,

$$
\begin{array}{ll}
\Gamma_{00}^{1}=\nu^{\prime} \epsilon^{2(\nu-\lambda)} & \Gamma_{10}^{0}=\nu^{\prime} \\
\Gamma_{11}^{1}=\lambda^{\prime} & \Gamma_{12}^{2}=\Gamma_{13}^{3}=1 / r \\
\Gamma_{22}^{1}=-r e^{-2 \lambda} & \Gamma_{23}^{3}=\cot \theta \\
\Gamma_{33}^{1}=-r \sin ^{2} \theta e^{-2 \lambda} & \Gamma_{33}^{2}=-\sin \theta \cos \theta
\end{array}
$$

3. The Ricci tensor is defined by

$$
R_{\mu \nu}=\Gamma_{\mu \alpha, \nu}^{\alpha}-\Gamma_{\mu \nu, \alpha}^{\alpha}-\Gamma_{\mu \nu}^{\alpha} \Gamma_{\alpha \beta}^{\beta}+\Gamma_{\mu \beta}^{\alpha} \Gamma_{\nu \alpha}^{\beta}
$$

Hence derive for the case under consideration,

$$
\begin{aligned}
& R_{00}=\left(-\nu^{\prime \prime}+\lambda^{\prime} \nu^{\prime}-\nu^{\prime 2}-\frac{2 \nu^{\prime}}{r}\right) e^{2(\nu-\lambda)} \\
& R_{11}=\nu^{\prime \prime}-\lambda^{\prime} \nu^{\prime}+\nu^{\prime 2}-\frac{2 \lambda^{\prime}}{r} \\
& R_{22}=\left(1+\tau \nu^{\prime}-r \lambda^{\prime}\right) e^{-2 \lambda}-1 \\
& R_{33}=R_{22} \sin ^{2} \theta
\end{aligned}
$$

4. Einstein made the assumption that in empty space outside a star

$$
R_{\mu \nu}=0
$$

Hence show from the vanishing of $R_{00}, R_{11}$ that

$$
\lambda^{\prime}+\nu^{\prime}=0
$$

(Do not confuse $\nu, \lambda$ when used to denote indices and when used to denote the metric functions.) For large $r$, space must be unaffected by the star and therefore flat, so that $\lambda, \nu$ tend to zero. So

$$
\lambda+\nu=0
$$


Hence show that

$$
\left(1+2 r \nu^{\prime}\right) e^{2 \nu^{\prime}}=1
$$

Show that this integrates to

$$
g_{00} \equiv e^{2 \nu}=1-\frac{2 M}{r}
$$

where $M$ is the constant of integration. By studying the Newtonian approximation one identifies it with the mass of the star. From the foregoing results show that,

$$
g_{11}=-\epsilon^{2 \lambda}=-e^{-2 \nu}=-\left(1-\frac{2 M}{r}\right)^{-1}
$$

This completes the derivation of the Schwarzschild solution of Einstein's equations outside a spherical static star. It was the first, and one of only three exact solutions that have been found. The second was found by Friedmann (1922) and is important for cosmology because it is a time dependent one. A third solution has been recently found by Senovilla, which unlike the other two has no singularity [112]. It may have profound implications for cosmology. It is the singularity of earlier solutions which led to the hypothesis of the 'big bang'.

\subsection{Stable Star Configurations}

Stability of stellar structures is a very important and complicated problem to analyse rigorously: Here we deal only with a qualitative treatment. Sketch the hydrostatic equilibrium curve for the mass of a neutron star as a function of its central density, showing the mass increasing to a maximum and then decreasing. Consider a star that lies on the portion of the curve where $\partial M / \partial \epsilon_{c}>0$. Imagine that the density of the star is perturbed by a small amount, so that it is shifted off the curve. Its mass is still $\mathrm{M}$ but its central density has changed. If it has been compressed it finds that its mass is too small for its new density. It will therefore expand since the gravitational attraction corresponding to this mass has been determined by the hypothetical hydrostatic equilibrium curve you have drawn to have a smaller density. Alternately, we may reason that the mass of the star that is in equilibrium at the perturbed density of our star $M$ is larger than $M$. So the gravitational force in our star will be too weak to maintain the compression at the perturbed value; it will expand to its former equilibrium configuration. Similarly if it has been perturbed in the opposite direction of the equilibrium curve, it will be impelled back. Therefore the portions of the equilibrium curve which have positive slope are stable. Use the same reasoning to find that the portions of negative slope are unstable, by showing that a perturbation that takes the star off the curve is subject to forces that move it further away. 


\section{References}

[1] C. W. Misner, K. S. Thorne and J. A. Wheeler, Gravitation Freeman \& Co., San Francisco, 1973.

[2] S. Weinberg, Gravitation and Cosmology, Wiley \& Sons, N. Y. 1972.

[3] P. A. M. Dirac, General Theory of Relativity, (Wiley, N. Y. 1975).

[4] N. K. Glendenning, T. Kodama and F. R. Klinkhamer, Phys. Rev. D 38 (1988) 3226.

[5] J. H. Taylor and J. M. Weisberg, Astrophys. J. 345 (1989) 434.

[6] J. M. Weisenberg and J. H. Taylor, Phys. Rev. Lett. 52 (1984) 1348;

J. H. Taylor (private communication, April 1987).

[7] J. M. Rankin, Astrophys. J. 274 (1983) 333; loc. cit. 301 (1986) 901.

[S] J. M. Rankin and J. A. Gil, Comments Astrophys. 14 (1989) 1.

[9] J. L. Friedman, J. A. Ipser and L. Parker, Phys. Rev. Lett. 62 (1989) 3015.

[10] N. K. Glendenning, Astrophys. J. 293 (1985) 470.

[11] B. K. Harrison, K. S. Thorne, M. Wakano and J. A. Wheeler, Gravitation theory and gratitational collapse, (Chicago: University of Chicago Press) 1965.

[12] J. W. Negele and D. Vautherin, Nucl. Phys. A178 (1973) 123.

[13] N. K. Glendenning, Nucl. Phys. A512 (1990) 737.

[14] I. S. Gradshteyn and I. M. Ryzhik, Tables of Integrals, Series and Products, Academic Press, N. Y., 1965.

[15] S. A. Chin, Plyys. Lett. 78B (1978) 552.

[16] J. I. Kapusta, Nucl. Phys. B148 (1979) 461.

[17] O. K. Kalashnikov and V. V. Klimov, Phys. Lett. 88B (1979) 328.

[18] R. F. Sawyer, Phys. Lett. 233 (1989) 412.

[19] C. W. Misner and H. S. Zapolsky, Phys. Rev. Lett. 12 (1964) 635.

[20] E. Witten, Phys. Rev. D 30 (1984) 272.

[21] E. Farhi and R. L. Jaffe, Phys. Rev. D 30 (1984) 2379. 
[22] S. I. A. Garpman, N. K. Glendenning and Y. J. Karant, Nuc. Phys. A322 (1979) 382.

[23] B. D. Serot and J. D. Walecka, Phys. Lett. 87B (1980).

[24] C. J. Horowitz and B. D. Serot, Nucl. Phys. A368 (1981) 413.

[25] J. Boguta, Nucl. Phys. A372 (1981) 386.

[26] P.-G. Reinhard, M. Rufa, J. Maruhn, W. Greiner and J. Friedrich, Z. Phys. A323 (1986) 13.

[27] B. D. Serot and J. D. Walecka, The Relativistic Nuclear Many-Body Problem, in "Advances in Nuclear Physics", eds. J. W. Negele and E. Vogt, (Plenum Press N. Y. ), 1986.

[28] N. K. Glendenning, Phys. Lett. 114B (1982) 392; Astrophys. J. 293 (1985) 470; Z. Phys. A 326 (1987) 5i; ibid. 327 (1987) 295; Nucl. Phys. A 493 (1989) 521 .

[29] J. D. Bjorken and S. D. Drell, Relativistic Ficlds, McGraw Hill, N. Y., 1965.

[30] N. K. Glendenning, Phys. Lett. 185B (1987) 275;

N. K. Glendenning, Nucl. Phys. A 469 (1987) 600 .

[31] C. H. Johnson, D. \&. Horen and C. Mahaux, Phys. Rev. C 36 (1987) 2252.

[32] J. P. Blaizot, D. Gogny and B. Grammiticos, Nucl. Phys. A265 (1976) 315;

J. P. Blaizot, Phys Rep. 64 (1980) 171.

[33] H. Krivine, J. Treiner and O. Bohigas, Nucl. Phys. A336 (1980) 155.

[34] N. K. Glendenning. Phys. Rev. Lett. 57 (1986) 1120.

[35] N. K. Glendenning, Phys. Rev. C 37 (1988) 2733.

[36] M. M. Sharma, W. T. A. Borghols, S. Brandenburg, S. Crona, A. van der Woude and M. N. Harakeh, Phys. Rev. C 38 (1988) 2562.

[37] V. R. Pandharipande, Nucl. Phys. A178 (1971) 123.

[38] H. A. Bethe and M. Johnson, Nucl. Phys. A230 (1974) 1.

[39] D. B. Kaplan and A. Nelson, Phys. Lett. 175 B (1986) 57.

[40] N. K. Glendenning, P. Hecking and V. Ruck, Ann. Phys. (N. Y.) 149 (1983) 22.

[41] N. K. Glendenning, Nucl. Phy's. A493 (1989) 521. 
[42] M. Prakash, T. L. Ainsworth and J. M. Lattimer, Phys. Rev. Lett. 61 (1988) 2518.

[43] P. C. Joss and S. A. Rappaport, Ann. Rev. of Astr. and Astrophys. 22 (1984) 537.

[44] B.Friedman, V. R. Pandharipande and Q. N. Usmani, Nucl. Phys. A372 (1981) 483.

[45] E. Baron, J. Cooperstein and S. Kahana, Nucl. Phys. A440 (1985) 744.

[46] E. Baron, J. Cooperstein and S. Kahana, Phys. Rev. Lett. 55 (1985) 126.

[17] S. E. Woosley and T. A. Weaver, Ann. Rev. Astron. Astrophys. 24 (1986) 205.

[48] S. E. Woolsey, P. A. Pinto, L. Ensman, Astrophys. J. 324 (1988) 466.

[49] E. Baron, H. A. Bethe, G. E. Brown, J. Cooperstein and S. Kahana, Phys. Rev. Lett. 59 (198i) 736.

[50] S. A. Bludman, Physics Reports 163 (1988) 47.

[51] N. K. Glendenning Comment on type II supernovae from prompt explosions LBL-24172 (unpublished, October, 1987).

[52] M. Takahara and K. Sato, Astrophys. J. 335 (1988) 301.

[53] H. A. Bethe, Ann. Rev. Nucl. and Part. Sc. 38 (1988) 1.

[54] K. A. Van Riper, Astrophy's. J.326 (1988) 235.

[55] E. S. Myra, S. A. Bludman, Y. Hoffman, 1. Lichtenstadt, N. Sack and K. A. Van Riper, Astrophys. J. 318 (1987) 744.

[56] S. W. Bruenn, Astrophys. J. 341 (1989) 385.

[57] J. Cooperstein and E. Baron, in Supernovae ed. by A. Petschek (SpringerVerlag, to appear, 1989).

[58] J. R. Wilson, in Numerical Astrophysics ed. by J. Centrella, J. LeBlanc and R. Bowers (Jones and Bartlett, Boston 1985) p422; J. R. Wilson, R. Mayle, S. E. Woosley and T. Weaver, Ann. New York Acadamy of Sciences 470 (1986) 267.

[59] J. Goodman, A. Dar and S. Nussinov, Astrophys. J. 314 (1987) L7.

[60] S. A. Colgate, in Supernovae, ed. by Wheeler et. al., World Scientific Press, Singapore, p. 249 (1990).

[61] S. A. Chin, Ann. Phys. (N.Y.) 108 (1977) 301. 
[62] C. J. Horowitz and B. D. Serot, Nucl. Phys. A464 (1987) 613.

[63] B. D. Serot and H. Uechi, Ann. Phys. (N. Y.) 179 (1987) 272.

[64] N. K. Glendenning, Nucl. Phys. A480 (1988) 597.

[65] J. Kunz, D. Masak and U. Post, Phys Lett. 186B (1987) 124.

[66] J. Boguta and A. R. Bodmer, Nucl. Phys. A292 (1977) 413.

[67] N. K. Glendenning, Phys. Lett. 114B (1982) 392.

[68] N. K. Glendenning, Z. Phys. A 326 (1987) 57.

[69] P. Möller, W. D. Myers, W. I. Swiatecki and J. Treiner, Atomic Data and Nuclear Data Tables, To be published (1988).

[70] B. D. Day and R. B. Wiringa, Phys. Rev. C 32 (1985) 1057.

[71] S. A. Moszkowski, Phys. Rev. D 9 (1974) 1613.

[72] G. E. Walker, Nucl. Phys. A450 (1986) 287c.

[73] V. A. Ambartsumyan and G. S. Saakyan, Astron. Zh. 37 (1963) 193 [Soviet Ast. - AJ,4 (1960) 187].

[74] N. K. Glendenning, Z. Phys. A, 327 (1987) 295.

[75] E. P. Mazets, S. V. Golenetski, R. L. Aptekar, Y. A. Guran and V. N. Illyinskii, Nature 290 (1981) 378.

[76] E. P. Liang, Astrophys. J. 304 (1986) 682.

[77] N. K. Glendenning, Supernovae, Compact Stars and Nuclear Physics, invited paper in Proc. of 1989 Int. Nucl. Phys. Conf., Sao Paulo, Brasil, Vol. 2, ed. by M. S. Hussein et al., World Scientific, Singapore, 1990.

[78] T. L. Ainsworth, E. Baron, G. E. Brown, J. Cooperstein, M. Prakash, Nucl. Phys. A464 (1987) 740.

[79] W. B. Fechner and P. C. Joss, Nature 274 (1978) 347.

[80] N. K. Glendenning, Strange-Quark-Matier Stars, invited paper, Rio de Janiero International Workshop on Relativistic Aspects of Nuclear Physics, August 1989, ed. by T. Kodama et al., World Scientific Publishing Co., Singapore, 1990.

[81] J. H. Taylor and D. R. Stinebring, Annu. Rev. Astron. Astrophys. 24 (1986) 285. 
[82] D. C. Backer, S. Kulkarni, C. Heiles, M. M. Davis, W. M. Goss, Nature 300 (1982) 615.

[83] J. M. Lattimer, M. Prakash, D. Masak and A. Yahil, Astrophys. J. bf 355 (1990) 241.

[8.1] N. K. Glendenning, Phys. Rev. Lett. 63 (1989) 2629.

[85] J. R. Ipser and L Lindbolm, Phys. Rev. Lett. 62 (1989) 2777.

[86] F. Weber, N. K. Glendenning and M. K. Weigel, Structure and Stability of Rotating Relativistic Neutron Stars, (LBL-28845, May 1990) Astrophys. J.

[87] F. Weber and N. K. Glendenning, Limiting Angular Velocity of Realistic Relativistic Neutron Star Models, (LBL-29141, June 1990) Zeits. fur Phys.

[88] N. k. Glendenning, J. of Phys. G, 15 (1989) L225.

[89] D. C. Backer, private communication, April, 1990.

[90] D. C. Backer and S. R. Kulkarni, Physics Today, 43 (1990) 26.

[91] B. K. Harrison and J. A. Wheeler, cited in B. K. Harrison et al. Gravitation Theory and Gravitational Collapse (University of Chicago Press, Chicago, 1965).

[92] C. Alcock and E. Farhi, Phys. Rev. D 32 (1985) 1273.

[93] A. R. Bodmer, Phỹs. Rev. D 4 (1971) 1601.

[94] C. Alcock, E. Farhi and A. V. Olinto, Astrophys. J. 310 (1986) 261.

[95] P. Haensel, J. L. Zdunik and R. Schaeffer, Astron. Astrophys. 160 (1986) 121.

[96] P. Haensel and J. L. Zdunik, Nature 340 (1989) 617.

[97] F. Grassi, accepted by Astrophys. J. (1990).

[98] J. A. Frieman and A. V. Olinto, Nature 341 (1989) 633.

[99] C. Alcock and A. V. Olinto, Ann. Rev. Nucl. Part. Sci. 38 (1988) 161.

[100] A. V. Olinto, Phy's. Lett. 192B (1987) 71.

[101] E. Cappellaro and M. Turatio, Astron. Astrophys. 190 (1988) 10.

[102] J. P. A. Clark and D. M. Eardley, Astrophys. J. 215 (1977) 311.

[103] A. G. Lyne, B. Anderson, and M. Salter, Mon. Not. R. Astron. Soc. 201 (1982) 503. 
[104] B. Smith, private communication.

[105] M. Brugger et al., Nature 337 (1989) 434.

[106] P. Braun Munziger, AGS Expt E814.

[107] H. J. Crawford et al., AGS Expt. P867 (1990).

[108] J. Sandweiss et al. AGS Expt. P864 (1990).

[109] H-C. Liu and G. L. Shaw, Phys. Rev. D 30 (1984) 30.

[110] C. Greiner, P. Kock and H. Stocker, Phys. Rev. Lett. 58 (1987) 1825.

[111] G. L. Shaw, M. Shin, R. H. Dalitz and M. Desai, Nature 337 (1989) 436.

[112] J. M. M. Senovilla, Phys. Rev. Lett. 64 (1990) 2219. 